UNIVERSIDADE DE SÃO PAULO

FACULDADE DE ECONOMIA, ADMINISTRAÇÃO E CONTABILIDADE DEPARTAMENTO DE ADMINISTRAÇÃO PROGRAMA DE PÓS-GRADUAÇÃO EM ADMINISTRAÇÃO

PROJEÇÃO DE PREÇOS DE ALUMÍNIO: MODELO ÓTIMO POR MEIO DE COMBINAÇÃO DE PREVISÕES

João Bosco Barroso de Castro

Orientadora: Profa. Dra. Alessandra de Ávila Montini 
Prof. Dr. Marco Antônio Zago

Reitor da Universidade de São Paulo

Prof. Dr. Adalberto Américo Fischmann

Diretor da Faculdade de Economia, Administração e Contabilidade

Prof. Dr. Roberto Sbragia

Chefe do Departamento de Administração

Prof. Dr. Moacir de Miranda Oliveira Júnior

Coordenador do Programa de Pós-Graduação em Administração 


\title{
PROJEÇÃO DE PREÇOS DE ALUMÍNIO: MODELO ÓTIMO POR MEIO DE COMBINAÇÃO DE PREVISÕES
}

\author{
Tese apresentada ao Departamento de \\ Administração da Faculdade de Economia, \\ Administração e Contabilidade da Universidade \\ de São Paulo como requisito para a obtenção do \\ título de Doutor em Ciências.
}

Orientadora: Profa. Dra. Alessandra de Ávila Montini

SÃO PAULO 
FICHA CATALOGRÁFICA

Elaborada pela Seção de Processamento Técnico do SBD/FEA/USP

\section{Castro, João Bosco Barroso de}

Projeção de preços alumínio: modelo ótimo por meio de combinação de previsões / João Bosco Barroso de Castro. - São Paulo, 2015.

$121 \mathrm{p}$.

Tese (Doutorado) - Universidade de São Paulo, 2015.

Orientador: Alessandra de Ávila Montini.

1. Previsão econômica 2. Combinação de previsões 3. Seleção de modelos 3. Preços de alumínio 4. Análise de série temporais I. Universidade de São Paulo. Faculdade de Economia, Administração e Contabilidade. II. Título.

CDD - 338.544 
Aos meus pais Lucimar e Monteiro, minha esposa Tania e aos meus filhos Isabelle, Fernanda e Victor 


\section{Agradecimentos}

Agradeço, especialmente, à minha orientadora, Profa. Dra. Alessandra de Ávila Montini, pela competência, confiança, sugestões, ensinamentos e amizade ao longo dessa jornada.

Ao Prof. Dr. Emérson Fernandes Marçal, meu co-orientador, pelas sugestão do Model Confidence Set, apoio e ensinamentos.

Ao Prof. Dr. Adolpho Walter P. Canton, pelo exemplo de mestre, sabedoria e dedicação.

Ao Prof. Dr. José Roberto Securato, pela dedicação e ensinamentos transmitidos durante o Mestrado e Doutorado.

Ao Prof. Naércio Menezes, por mostrar a intuição atrás dos números e conselhos.

Ao Prof. Dr. Fernando Almeida, pela amizade e incentivo.

Ao Wagner Monteiro, monitor das aulas de laboratório na FGV/SP, pela dedicação e entusiasmo.

Aos meus colegas de pós-graduação da FEA/USP e da FGV/SP.

À minha cunhada Kátia, pela amizade e convivência.

Aos meus irmãos Monteiro, Joaquim e Alba, pelo carinho e apoio constante. 


\section{RESUMO}

Commodities primárias, tais como metais, petróleo e agricultura, constituem matérias-primas fundamentais para a economia mundial. Dentre os metais, destaca-se o alumínio, usado em uma ampla gama de indústrias, e que detém o maior volume de contratos na London Metal Exchange (LME). Como o preço não está diretamente relacionado aos custos de produção, em momentos de volatilidade ou choques econômicos, o impacto financeiro na indústria global de alumínio é significativo. Previsão de preços do alumínio é fundamental, portanto, para definição de política industrial, bem como para produtores e consumidores. Este trabalho propõe um modelo ótimo de previsões para preços de alumínio, por meio de combinações de previsões e de seleção de modelos através do Model Confidence Set (MCS), capaz de aumentar o poder preditivo em relação a métodos tradicionais. A abordagem adotada preenche uma lacuna na literatura para previsão de preços de alumínio. Foram ajustados 5 modelos individuais: AR(1), como benchmarking, ARIMA, dois modelos ARIMAX e um modelo estrutural, utilizando a base de dados mensais de janeiro de 1999 a setembro de 2014. Para cada modelo individual, foram geradas 142 previsões fora da amostra, 12 meses à frente, por meio de uma janela móvel de 36 meses. Nove combinações de modelos foram desenvolvidas para cada ajuste dos modelos individuais, resultando em 60 previsões fora da amostra, 12 meses à frente. A avaliação de desempenho preditivo dos modelos foi realizada por meio do MCS para os últimos 60, 48 e 36 meses. Um total de 1.250 estimações foram realizadas e 1.140 variáveis independentes e suas transformadas foram avaliadas. A combinação de previsões usando ARIMA e um ARMAX foi o único modelo que permaneceu no conjunto de modelos com melhor acuracidade de previsão para 36, 48 e 60 meses a um nível descritivo do MCS de 0,10. Para os últimos 36 meses, o modelo combinado proposto apresentou resultados superiores em relação a todos os demais modelos. Duas co-variáveis identificadas no modelo ARMAX, preço futuro de três meses e estoques mundiais, aumentaram a acuracidade de previsão. A combinação ótima apresentou um intervalo de confiança pequeno, equivalente a 5\% da média global da amostra completa analisada, fornecendo subsídio importante para tomada de decisão na indústria global de alumínio. 


\section{ABSTRACT}

Primary commodities, including metals, oil and agricultural products are key raw materials for the global economy. Among metals, aluminum stands out for its large use in several industrial applications and for holding the largest contract volume on the London Metal Exchange (LME). As the price is not directly related to production costs, during volatility periods or economic shocks, the financial impact on the global aluminum industry is significant. Aluminum price forecasting, therefore, is critical for industrial policy as well as for producers and consumers. This work has proposed an optimal forecast model for aluminum prices by using forecast combination and the Model Confidence Set for model selection, resulting in superior performance compared to tradicional methods. The proposed approach was not found in the literature for aluminum price forecasting. Five individual models were developed: AR(1) for benchmarking, ARIMA, two ARIMAX models and a structural model, using monthly data from January 1999 to September 2014. For each individual model, 142 out-of-sample, 12 month ahead, forecasts were generated through a 36 month rolling window. Nine foreast combinations were deveoped for each individual model estimation, resulting in 60 out-ofsample, 12 month ahead forecasts. Model predictive performace was assessed through the Model Confidence Set for the latest 36, 48, and 60 months, through 12-month ahead out-ofsample forecasts. A total of 1,250 estimations were performed and 1,140 independent variables and their transformations were assessed. The forecast combination using ARMA and ARIMAX was the only model among the best set of models presenting equivalent performance at 0.10 MCS p-value in all three periods. For the latest 36 months, the proposed combination was the best model at 0.1 MCS p-value. Two co-variantes, identified for the ARMAX model, namely, 3-month forward price and global inventories increased forecast accuracy. The optimal forecast combination has generated a small confidence interval, equivalent to $5 \%$ of average aluminum price for the entire sample, proving relevant support for global industry decision makers. 


\section{SUMÁRIO}

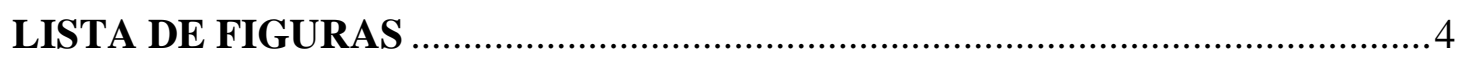

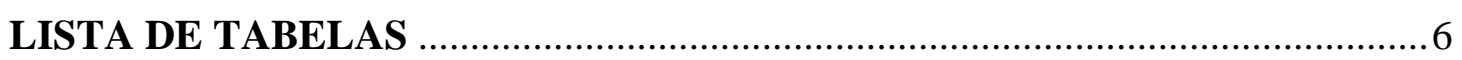

1 INTRODUÇÃ

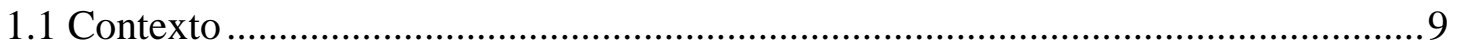

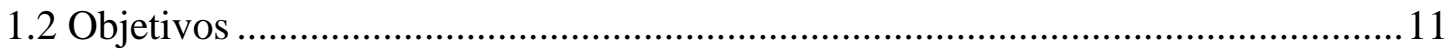

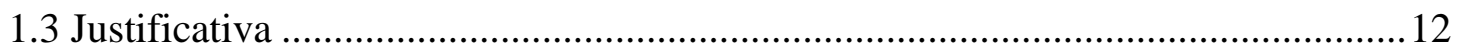

1.4 Estrutura da Tese......................................................... 14

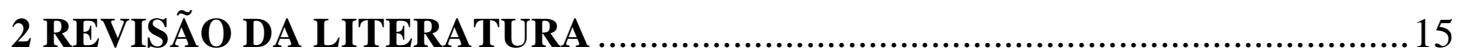

2.1 Modelos de Previsão de Commodities.........................................15

2.3 Modelos Individuais Utilizados na Previsão de Preços de Alumínio..............19

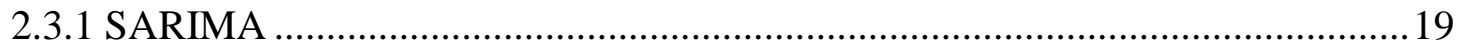

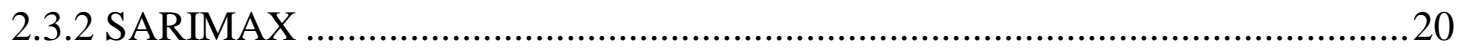

2.3.3 Modelo Estrutural.....................................................20

2.4 Seleção de Modelos..................................................21

2.4.1 Abordagem Tradicional...................................................21

2.4.2 O Método de Diebold e Mariano...........................................23

2.4.3 Model Confidence Set (MCS) .......................................24

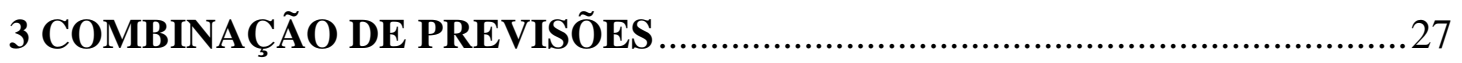

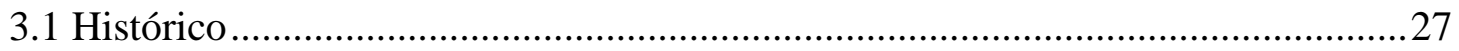

3.2 O Problema de Combinação de Previsões.......................................33

3.3 Combinação de Previsões sob Perda do Erro Quadrático Médio....................35

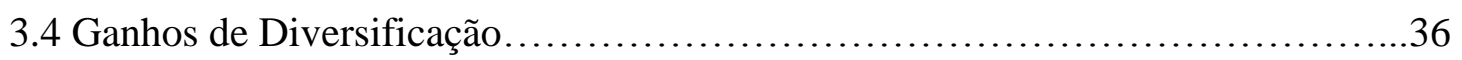

3.5 Métodos de Combinação de Previsões........................................40

3.5.1 Combinações Simples..............................................40

3.5.1.1 Média Simples...................................................40

3.5.1.2 Mediana............................................................41

3.5.1.3 Média Aparada...........................................................41

3.5.2 Método da Raiz do Erro Quadrático Médio (RMSE) .......................42

3.5.3 Método do Critério de Informação de Akaike (AIC) ........................42

3.5.4 Método de Mínimos Quadrados Ordinários (OLS) ..........................43

3.5.5 Intervalo de Confiança de Combinação pelo Método de Mínimos Quadrados

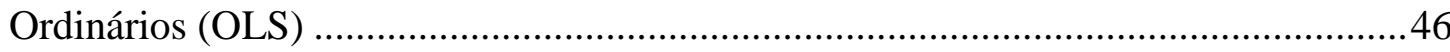


3.5.6 Métodos de Mínimos Quadrados Restritos..................................48

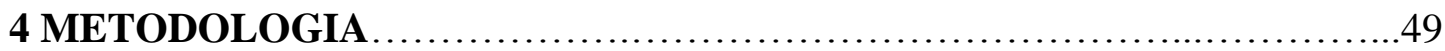

4.1 Variável Dependente...................................................49

4.1.1 Série Original de Preço à Vista de Alumínio (Valores Nominais)..............49

4.1.2 Série de Preço à Vista de Alumínio a Valores Constantes.......................51

4.1.3 Série Transformada de Preço de Alumínio..................................54

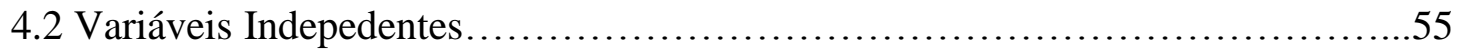

4.3 Amostras Utilizadas............................................................56

4.3.1 Amostras Utilizadas para Seleção dos Modelos Individuais ...................56

4.3.2 Amostras Utilizadas.para Ajustes dos Modelos e Projeções.....................56

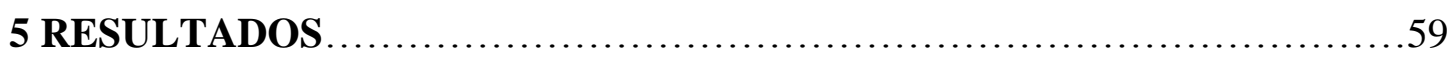

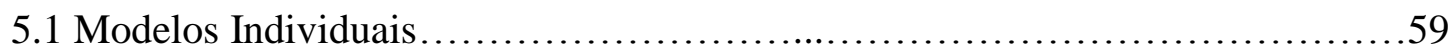

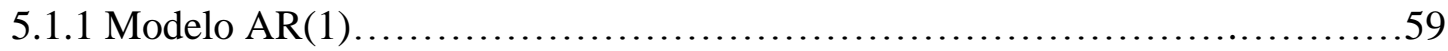

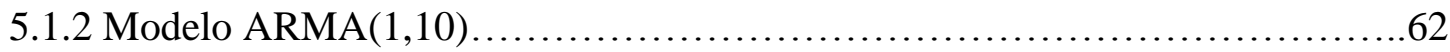

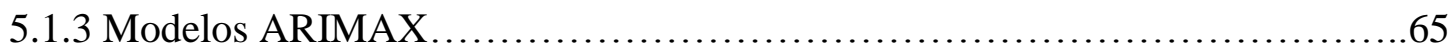

5.1.3.1 Suposições para Especificação do Modelo ARIMAX ......................65

5.1.3.2 Teste de Estacionariedade das Variáveis Independentes do Modelo ARIMAX ...............................................................66

5.1.3.3 Teste de Exogeneidade Fraca das Varáveis Independentes para o Modelo ....67

5.1.3.4 Teste de Causalidade de Granger......................................70

5.1.3.5 Modelos ARIMAX propostos........................................71

5.1.3.5.1 Modelo ARIMAX 1....................................................71

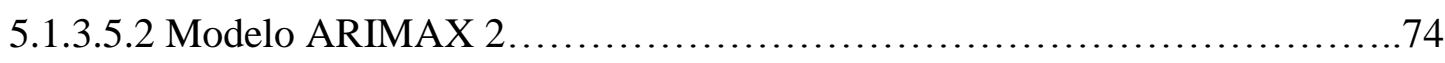

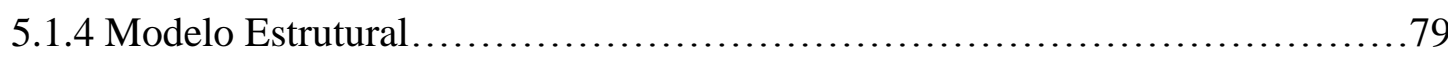

5.2 Modelos Combinados................................................ 83

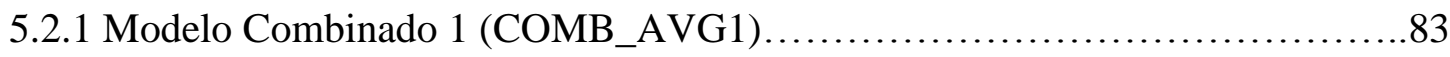

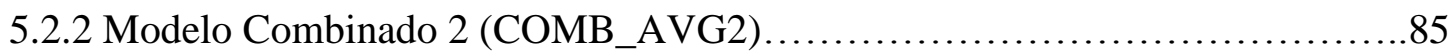

5.2.3 Modelo Combinado 3 (COMB_OLS) ................................. 87

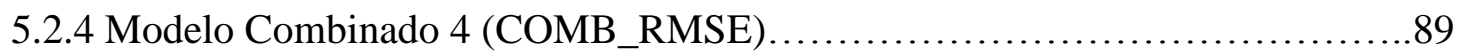

5.2.5 Modelo Combinado 5 (COMB_AICC) ..................................... 91

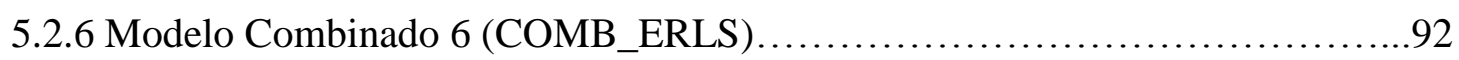

5.2.7 Modelo Combinado 7 (COMB_NERLS) ................................. 94

5.2.8 Modelo Combinado 8 (COMB_NRLS) .............................. 96

5.2.9 Modelo Combinado $9($ COMB_ARMAX) ................................... 97 


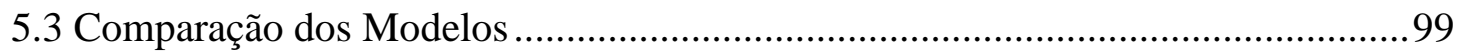

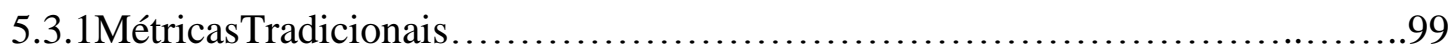

5.3.2 Comparação de Modelos por meio do Model Confidence Set...................101

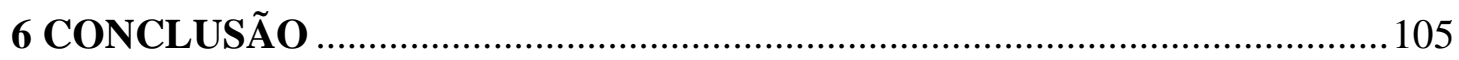

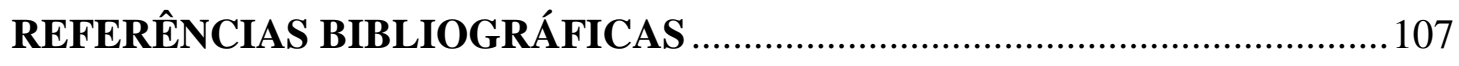

ANEXO A - Programas SAS - Combinações de Modelos …………………….......112

ANEXO B - Programas SAS - Geração de Modelos Individuais ...........................117 


\section{LISTA DE FIGURAS}

Figura 1 - Série Original do Preço à Vista de Alumínio .............................................50

Figura 2 - Histograma do Preço à Vista de Alumínio ..................................................50

Figura 3 - Evolução do Preço à Vista de Alumínio .....................................................51

Figura 4 - Séries original e deflacionadas pelo CPI, PPI e NF .................................52

Figura 5 - Séries de preço à vista de alumínio em valores nominais e constantes .....52

Figura 6 - Função de Autocorrelação e Autocorrelação Parcial .................................53

Figura 7 - Série Transformada do Retorno do Preço de Alumínio ................................54

Figura 8 - Abordagem para Estimação e Projeção do Preço de Alumínio.....................57

Figura 9 - Análise de Correlação dos Resíduos do Modelo AR(1)..............................60

Figura 10 - Análise de Normalidade dos Resíduos do Modelo AR(1) ........................61

Figura 11 - Modelo AR(1) Ajustado dentro da Amostra Completa ...........................61

Figura 12 - Modelo AR(1): Preços de Alumínio Observados e Projetados ...................62

Figura 13 - Análise de Correlação dos Resíduos do Modelo ARMA $(1,10)$...............63

Figura 14 - Análise de Normalidade dos Resíduos do Modelo ARMA(1, 10)............64

Figura 15 - Modelo ARMA $(1,10)$ Ajustado dentro da Amostra Completa ................64

Figura 16 - Modelo ARMA(1, 10): Preços de Alumínio Observados e Projetados ....65

Figura 17 - Análise de Correlação dos Resíduos do Modelo ARIMAX 1 ................... 72

Figura 18 - Análise de Normalidade dos Resíduos do Modelo ARIMAX 1 ................72

Figura 19 - Modelo ARIMAX 1 Ajustado dentro da Amostra Completa ....................73

Figura 20 - Modelo ARIMAX 1: Preços Observados e Projetados...................74

Figura 21 - Evolução do preço à vista e estoque mundial de alumínio...............75

Figura 22 - Análise de Correlação dos Resíduos do Modelo ARIMAX 2 ................... 77

Figura 23 - Análise de Normalidade dos Resíduos do Modelo ARIMAX 2 ................77

Figura 24 - Modelo ARIMAX 2 Ajustado para a estimação mais recente .............78

Figura 25 - Modelo AR1MAX 2: Preços Observados e Projetados ..................79

Figura 26 - Análise de Correlação dos Resíduos do Modelo Estrutural ........................80

Figura 27 - Análise de Normalidade dos Resíduos do Modelo Estrutural....................81

Figura 28 - Modelo Estrutural Ajustado dentro da Amostra Completa ........................ 81

Figura 29 - Modelo Estrutural: Preços Observados e Projetados.....................82

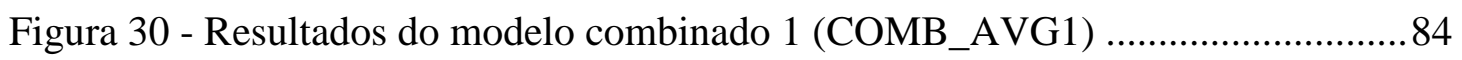

Figura 31 - Evolução do RMSE do modelo combinado 1 (COMB_AVG1) .................85

Figura 32 - Resultados do modelo combinado 2 (COMB_AVG2) .............................86 
Figura 33 - Evolução do RMSE do modelo combinado 2 (COMB_AVG2) ................87

Figura 34 - Resultados do modelo combinado 3 (COMB_OLS)................................. 88

Figura 35 - Evolução do RMSE do modelo combinado 3 (COMB_OLS) ...................88

Figura 36 - Resultados do modelo combinado 4 (COMB_RMSE) .............................89

Figura 37 - Evolução do RMSE do modelo combinado 4 (COMB_RMSE) ...............90

Figura 38 - Resultados do modelo combinado 5 (COMB_AICC) ................................91

Figura 39 - Evolução do RMSE do modelo combinado 5 (COMB_AICC) .................92

Figura 40 - Resultados do modelo combinado 6 (COMB_ERLS) .............................93

Figura 41 - Evolução do RMSE do modelo combinado 6 (COMB_ERLS) ................. 93

Figura 42 - Resultados do modelo combinado 7 (COMB_NERLS) ...........................94

Figura 43 - Evolução do RMSE do modelo combinado 7 (COMB_NERLS) ..............95

Figura 44 - Resultados do modelo combinado 8 (COMB_NRLS) ..............................96

Figura 45 - Evolução do RMSE do modelo combinado 8 (COMB_NRLS)................97

Figura 46 - Resultados do modelo combinado 9 (COMB_ARMAX) ........................98

Figura 47 - Evolução do RMSE do modelo combinado 9 (COMB_ARMAX) ........... 98

Figura 48 - Evolução de dias de estoques globais de alumínio e taxas de juros ......... 103 


\section{LISTA DE TABELAS}

Tabela 1 - Estatísticas descritivas da série original de preço de alumínio ....................51

Tabela 2 - Teste de Portmanteau para a Série de Preço Deflacionado de Alumínio ...53

Tabela 3 - Teste de Raiz Unitária ADF ..................................................................53

Tabela 4 - Teste de Raiz Unitária ADF para a Série Transformada ....................54

Tabela 5 - Variáveis Independentes Analisadas..........................................................5

Tabela 6 - Total de transformações das variáveis independentes ...............................56

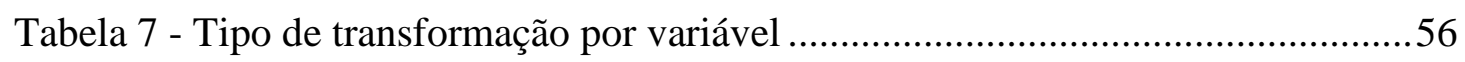

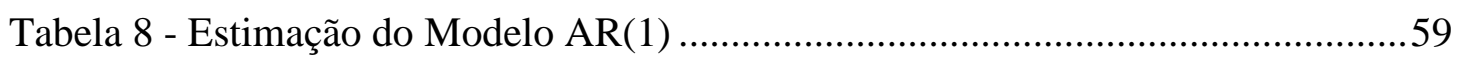

Tabela 9 - Estatísticas de Ajuste do Modelo AR(1)....................................................60

Tabela 10 - Análise de Normalidade dos Resíduos do Modelo AR(1) .......................61

Tabela 11 - Modelos ARMA com Menores Critérios de Informação (AIC, SBC) .....61

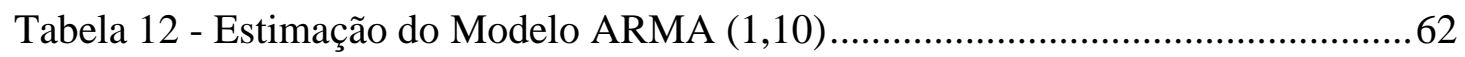

Tabela 13 - Estatísticas de Ajuste do Modelo ARMA(1, 10) ...................................63

Tabela 14 - Análise de Normalidade dos Resíduos do Modelo ARMA(1,10)............64

Tabela 15 - Variáveis Independentes Transformadas e Estacionárias ..........................67

Tabela 16 - Teste de Exogeneidade Fraca para Variáveis Independentes ....................68

Tabela 17 - Teste de Exogeneidade Fraca para Variáveis Independentes ..................69

Tabela 18 - Teste de Causalide de Granger para Variáveis Independentes .................70

Tabela 19 - Estimação do Modelo ARIMAX 1 ........................................................ 71

Tabela 20 - Estatísticas de Ajuste do Modelo ARIMAX 1.........................................71

Tabela 21 - Análise de Normalidade dos Resíduos do Modelo ARMAX 1 ................73

Tabela 22 - Estimação do Modelo ARIMAX 2 …........................................................ 76

Tabela 23 - Estatísticas de Ajuste do Modelo ARIMAX 2 2.........................................76

Tabela 24 - Análise de Normalidade dos Resíduos do Modelo ARMAX 2 …............77

Tabela 25 - Estimação do Modelo Estrutural............................................................... 79

Tabela 26 - Estatísticas de Ajuste do Modelo Estrutural .............................................. 80

Tabela 27 - Análise de Normalidade dos Resíduos do Modelo Estrutural...................81

Tabela 28 - Descrição dos Modelos Combinados....................................................... 83

Tabela 29 - Métricas de acuracidade fora da amostra do modelo combinado 1 ......85

Tabela 30 - Métricas de acuracidade fora da amostra do modelo combinado 2 .......87

Tabela 31 - Métricas de acuracidade fora da amostra do modelo combinado 3.......89

Tabela 32 - Métricas de acuracidade fora da amostra do modelo combinado 4 ......90 
Tabela 33 - Métricas de acuracidade fora da amostra do modelo combinado 5.......92

Tabela 34 - Métricas de acuracidade fora da amostra do modelo combinado 6.......94

Tabela 35 - Métricas de acuracidade fora da amostra do modelo combinado 7.......95

Tabela 36 - Métricas de acuracidade fora da amostra do modelo combinado 8.......97

Tabela 37 - Métricas de acuracidade fora da amostra do modelo combinado 9.......99

Tabela 38 - Comparação de Modelos - 36 meses mais recentes ................................ 99

Tabela 39 - Comparação de Modelos - 48 meses mais recentes ............................... 100

Tabela 40 - Comparação de Modelos - 60 meses mais recentes ................................ 100

Tabela 41 - Comparação dos modelos por meio do MCS ........................................ 102 


\section{INTRODUÇÃO}

\subsection{Contexto}

Commodities primárias, tais como metais, petróleo e de agricultura, constituem matériasprimas fundamentais dos processos produtivos da economia mundial. Dentre os metais, o alumínio destaca-se por ser o elemento mais abundante na crosta terrestre $(8,1 \%)$. Após o oxigênio e o silício, é o terceiro elemento (entre metálicos e não metálicos) mais abundante. Apesar de ser um metal relativamente recente (com pouco mais de 100 anos desde sua primeira comercialização), o seu uso supera o de qualquer outro metal, exceto aço. O alumínio é usado virtualmente em todos os segmentos da economia mundial, desde transportes, construção, setor elétrico até embalagens, equipamentos e indústria aeroespacial. Caracteriza-se por ser um produto leve (um terço da densidade do aço), anti-corrosivo, flexível, de boa condutividade elétrica ( $1 \mathrm{~kg}$ de alumínio conduz a mesma quantidade de eletricidade que $2 \mathrm{~kg}$ de cobre), refletor de calor irradiante, transmissor de calor por convecção, além de ser 100\% reciclável. A sua principal fonte de obtenção é a bauxita, que necessita ser transformada em alumina, a partir da qual se produz alumínio.

O Brasil destaca-se na indústria global do alumínio uma vez que reúne características para isso: possui a terceira maior reserva de bauxita, é o terceiro maior produtor de bauxita e alumina e o sexto maior produtor de alumínio no mundo. O faturamento da indústria brasileira do alumínio em 2013, incluindo a contabilização de todas as etapas da cadeia de valor, desde a mineração até a reciclagem do metal, cresceu 12,6\% em 2013 e somou $\mathrm{R} \$ 39,2$ bilhões. O montante foi $\mathrm{R} \$ 4,4$ bilhões a mais que o registrado no ano de 2012 (R \$ 34,8 bilhões). Com esse resultado, a participação do setor de alumínio no PIB Industrial do País tornou-se mais substancial, ao atingir 3,8\% (no exercício anterior havia ficado em 3,6\%). Os investimentos do setor totalizaram $\mathrm{R} \$ 3$ bilhões. O pagamento de impostos, que avançou 14,3\%, fechou 2013 com R \$ 6,4 bilhões.

Depois de registrar 1.440,8 mil toneladas em 2012, o consumo doméstico de produtos transformados de alumínio cresceu 5\% e fechou o ano passado em 1.512,5 mil toneladas. Significativos são também os números da balança comercial. Enquanto as exportações da indústria brasileira de alumínio caíram 8,4\%, reduzindo, com isso, o valor total FOB para US\$ 
3.575 milhões no ano passado (US\$ 3.902 milhões em 2012), as importações foram reduzidas em apenas $0,7 \%$, caindo de US\$ 1.355 milhões para US\$ 1.346 milhões.

O alumínio pode ser reciclado infinitamente, em qualquer forma em que se apresente, de sobras do processo de fabricação a produtos com a vida útil esgotada. Em 2012 o Brasil reciclou 267,1 mil toneladas de latas de alumínio para bebidas, das 272,8 mil toneladas disponíveis no mercado. No total foram reaproveitadas 19,8 bilhões de embalagens, o correspondente a 54,1 milhões/dia, ou 2,3 milhões/hora. Com estes volumes, o índice brasileiro de reciclagem do produto ficou em 97,9\%. Dentre as nações mais ricas do mundo, a única que se aproximou desse percentual foi o Japão, com 94,7\%, enquanto os Estados Unidos reciclaram $67 \%$.

O alumínio é uma commodity negociada no mundo inteiro, com base na cotação diária da London Metal Exchange (LME), principal bolsa mundial para metais não ferrosos, e que introduziu em 1978 contratos à vista e a termo (3 meses). O alumínio representa o maior contrato da LME, tanto em contratos futuros como opções. Como o preço não está diretamente relacionado aos custos de produção, em momentos de volatilidade de mercado ou de choques econômicos, o impacto na indústria global pode ser significativo.

A crise financeira global de 2008/2009 causou uma queda do preço ao alumínio de cerca de US\$ 3.071 por tonelada em julho de 2008 para \$1.330 por tonelada em fevereiro de 2009. Ao mesmo tempo, os estoques mundiais de alumínio aumentaram continuamente, passando de 11,4 milhões de toneladas em julho de 2008 para 46 milhões de toneladas em setembro de 2009. Esta situação levou ao corte de capacidade produtiva de 3 milhões de toneladas em 2008/2009, sendo 1,9 milhões de toneladas na China e 1,1 milhões de toneladas na Europa e Estados Unidos. Em dezembro de 2011, os estoques ainda continuavam no patamar elevado de 47,9 milhões de toneladas e o preço em torno de US\$2.000 por tonelada. Desde então, os preços continuam baixos, em torno de US\$1,850 por tonelada, em comparação a uma média histórica de 11 anos (2000-2011) de US\$ 2.500/tonelada, a valores constantes de 2010. A indústria voltou a fazer cortes em 2010 e houve fechamento adicional de capacidade de 2011 a 2014.

A possibilidade de que preços sejam frequentemente aleatórios introduz uma parcela importante de risco e incerteza no processo de análise de mercado e de previsão do preço do alumínio. Dessa forma, a previsão de preços de alumínio é de extrema relevância dado seu 
impacto na cadeia industrial e sua volatilidade de preços. Esta aleatoriedade pode ser melhor compreendida a partir de sua dinâmica de curto, médio e longo prazo.

No curto prazo, choques de mercado tendem a estar associado a fatores financeiros, principalmente à especulação e hedging em mercados futuros, opções e derivativos; uma ampla literatura existe para análise no curto prazo, incluindo séries temporais para dados de alta frequência (BARKOULAS; LABYS, ONOCHIE, 1997; ADAMS; VIAL, 1988). No médio prazo, previsão de preços e sua interrelação com ciclos da indústria, influenciados por forças de mercado e condições econômicas nacionais, têm sido aspectos preponderantes. Métodos econométricos utilizam análise espectral e de séries temporais, incluindo modelos estruturais de séries de tempo, os quais incorporam análise de componentes cíclicos (HARVEY, 1989). No longo prazo, análise de tendência e de cenários de preços são importantes para avaliar investimentos de longa maturação de uma indústria de base, acima de 20 anos. Métodos econométricos de interesse têm incluído choques estruturais, subidas e quedas bruscas de preços e movimentos de reversão (CASHIN; MCDERMOTT, 2002; ANDREWS, 1993; PERRON, 1989).

\subsection{Objetivos}

Esta tese tem como objetivo principal propor um modelo ótimo de previsões para preços de alumínio, por meio de combinações de previsões e de seleção de modelos através do Model Confidence Set, capaz de aumentar o poder preditivo em relação a métodos tradicionais relatados na literatura. A abordagem adotada preenche uma lacuna na literatura e avança na análise de previsão de preços de alumínio no horizonte de doze meses.

Os objetivos específicos são os seguintes:

- $\quad$ Ajustar diferentes modelos de previsão de preços de alumínio;

- Determinar a combinação ótima das previsões dos modelos ajustados;

- Comparar a acuracidade de previsão dos modelos individuais e combinados. 


\subsection{Justificativa}

Em vista do contexto exposto, a modelagem e previsão de preços de alumínio constitui um tema relevante para o direcionamento de questões de política industrial de governos, decisões críticas de negócio para produtores (como decisões de investimentos) e consumidores (como estratégias de suprimento de matérias-primas), bem como para participantes do mercado financeiro, incluindo grandes instituições financeiras, gestores de portfólio e investidores individuais, visando melhorar o desempenho da relação risco-retorno.

Dessa forma, um entendimento melhor da dinâmica e previsão de preços de alumínio é extremamente desejável, tanto do ponto de vista de diretriz econômica, quanto na ótica dos produtores e consumidores.

Muitas pesquisas têm sido realizadas para previsão de preços de commodities utilizando diversas técnicas de séries temporais, como resposta aos complexos problemas relacionados à formação de preços de commodities. Componentes financeiros, tais como demanda especulativa e hedging, passaram, cada vez mais, a interferir no processo de formação de preços desses ativos, além dos fundamentos de mercado de oferta e demanda, amplificando a volatidade dos preços.

Pesquisas de preços de commodities remontam aos trabalhos de Slutsky (1927) e Yule (1927), que sugeriram que equações de diferenças, incluindo choques estocásticos, poderiam ser empregadas para modelar e prever séries temporais econômicas. Já havia uma ênfase nas abordagens em projetar tanto o nível quanto quebras estruturais dos preços (LEUTHOLD et al., 1970; DRIEHUIS, 1976). Tais abordagens foram conduzidas pelo Banco Mundial, conforme descrito por Duncan (1984) e Labys e Pollack (1984). Kouassi, Labys e Colyer (1998) mostraram como modelos univariados podem ser aplicados para commodities. Labys e Kouassi (2004) desenvolveram modelos estruturais para previsão de curto e médio prazos, com identificação de ciclos e quebras estruturais. Vários autores desenvolveram trabalhos utilizando técnicas de séries temporais para prever ciclos, como Harvey e Jaeger (1993) e Mills (2003). 
No entanto, as pesquisas desenvolvidas para previsão de preços de alumínio apresentam lacunas importantes. Primeiro, os estudos encontrados na literatura empregaram abordagens de previsão e de seleção de modelos tradicionais na previsão de preços de alumínio. Masoud (1988) fez um estudo comparativo de um modelo estrutural com um modelo multivariado para a indústria de cobre. Para comparar a performance preditiva desses modelos foram utilizados indicadores tradicionais (MAE, MAPE, RMSE, RMSPE). Sow (1996) utilizou um modelo estrutural multivariado para fazer previsões de preços de alumínio, cobre, zinco e estanho até 2011. Também utilizou modelos de curva de aprendizado para desenvolver as previsões, mas não realizou análise comparativa dos modelos. Dooley e Lenihan (2005) desenvolveram um modelo ARIMA para projeção de preços de zinco e estanho. No Brasil, Baltar (2009) desenvolveu previsão de preços de cobre utilizado modelo ARIMA. Mais recentemente, Ren e Ru (2012) também utilizaram um modelo ARMA para previsão de preços de alumínio.

Segundo, um problema fundamental diz respeito à seleção de modelos de previsão. As abordagens tradicionais baseiam-se em medidas relacionadas aos erros de previsão e selecionam apenas um modelo como sendo o de melhor desempenho preditivo. No entanto, tais medidas podem levar a conclusões errôneas decorrente do fato de existir apenas uma realização do processo estocástico. Um modelo pode ter um desempenho de previsão aparentemente superior a outro simplesmente por um resultado do acaso do que, de fato, apresentar melhor acuracidade de previsão. Tal fato está associado ao problema de data snooping, que ocorre quando um conjunto de dados é usado mais de uma vez para fins de inferência e de seleção de modelos (WHITE, 2000). Diferentemente dos modelos convencionais, o Model Confidence Set (MCS), proposto por Hansen, Lunde e Nason (2011), é capaz de fazer comparações múltiplas de modelos com base em um dado nível descritivo, levando em conta as limitações dos dados. Tal fato possibilita a seleção de um conjunto de modelos que podem apresentar acuracidade de previsão equiparável a um nível descritivo definido pelo MCS. Na revisão da literatura, não foi encontrada a aplicação do MCS para preços de alumínio. Entre poucos trabalhos, encontrou-se a aplicação do MCS para comparação de modelos de volatilidade por Laurent e Violante (2012), voltada para a taxa de câmbio EUR/USD.

Terceiro, não foram encontrados na literatura trabalhos que abordem, concorrentemente, previsão de preços de alumínio por meio de combinações de previsões e de seleção de modelos pelo Model Confidence Set. Desde o trabalho pioneiro de Granger e Bates (1969), vários 
estudos demonstram que combinações de previsões proporcionam resultados superiores em relação a modelos individuais, conforme Nelson (1972), Newbold e Granger (1974), Makridakis e Winkler (1983), Hendry e Clements (2002), Pesaran e Timmermann (2005), Stock e Watson (2006), Bruggemman e Lutkepohl (2013) e Hsiao e Wan (2014). Com relação a preços relacionados a commodities metálicas, um dos poucos trabalhos que se aproximaram dessa linha foi o de Issler e Rodrigues (2012), que desenvolveram análise comparativa de modelos para previsão do Metals Price Index (do Fundo Monetário Internacional), utilizando modelos AR, GARCH e VAR, bem como combinações de previsões e um modelo de correção de viés. No entanto, não foi utilizado o MCS e a maioria dos modelos eram variações de VAR.

Tendo em vista esses fatos, vê-se a necessidade de avançar no estudo de previsões de preços de alumínio por meio de combinações de modelos e aumentar a acuracidade dos resultados. Ao mesmo tempo, observa-se a importância de utilizar um método de seleção de modelos capaz de tratar efeitos de data snooping, como o MCS, com o intuito de se obter um intervalo de previsão de preços com maior grau de acuracidade em relação aos métodos convencionais. Tais pontos, no contexto da previsão de preços de alumínio, compõem o tema de pesquisa deste trabalho e preenchem uma lacuna na literatura.

\subsection{Estrutura da Tese}

A tese está estruturada da seguinte forma: o presente capítulo é introdutório e descreve os objetivos, justificativa do trabalho. O segundo capítulo aborda a revisão da literatura, incluindo o contexto de commodities metálicas, ajuste de modelos individuais e seleção de modelos. $\mathrm{O}$ terceiro capítulo aborda histórico e aspectos teóricos de combinação de previsões. O quarto capítulo inclui metodologia, descrição e tratamento de dados. O quinto capítulo apresenta os resultados obtidos, incluindo modelos individuais, combinações de previsões e comparações dos modelos individuais e combinados. O sexto capítulo conclui, com foco nas contribuições do trabalho e sugestões de estudos futuros. 


\section{REVISÃO DA LITERATURA}

\subsection{Modelos de Previsão de Commodities}

Historicamente, tem-se abordado a necessidade de prever níveis de preços de commodities, como também quebras estruturais ou pontos de inflexão dos preços, conforme Leuthold et al. (1970) e Driehuis (1976). Estudos de previsão de preços de commodities têm sido conduzidos pelo Banco Mundial, segundo Duncan (1984) e Labys e Pollack (1984). Uma revisão sobre como diferentes métodos univariados e multivariados podem ser aplicados à previsão de preços de minérios e metais foi realizada pela Organização das Nações Unidas (ONU) em 1984. Kouassi et al. (1998) também mostrou como modelos univariados e multivariados podem ser aplicados à previsão de preços de commodities. A avaliação da qualidade de previsão de modelos não estruturais tem sido objeto de vasta pesquisa, como indicado por Granger (1989).

No tocante aos principais metais industriais e ligas metálicas, os preços praticados baseiam-se em alguma forma de referência de mercado, principalmente através de bolsas. As bolsas atuam para proporcionar liquidez. A maioria das bolsas operam dentro de vários princípios, entre os quais se destacam:

- Estocagem: proporciona a entrega de produto, relacionando o preço ou índice da bolsa ao preço no mercado físico;

- Padronização: define as especificações do produto para garantir a validade da transação;

- Sistema de liquidação: faz a liquidação entre contratos de compra e venda, gerando liquidez necessária aos participantes de mercado;

- Integridade: por meio de um sistema de licenciamento para distribuidores registrados e que utiliza garantias das contra-partes das transações.

Em geral, as quatro especificações de entrega para metais são: data, localização, composição e formato do metal. Como os vendedores podem sempre ter a opção de realizar o preço de liquidação através da bolsa, os compradores de um determinado metal devem normalmente pagar um prêmio físico, que reflete a diferença entre o valor do produto entregue aos 
compradores e o valor de liquidação. O prêmio varia conforme as condições de mercado, sendo normalmente cerca de $1 \%$ do preço do metal, mas pode chegar a mais de $10 \%$.

O sistema de entrega e de armazenamento suporta o funcionamento da bolsa de metais. A quantidade, localização e especificação do metal nas localidades listadas pela bolsa constituem uma informação fundamental para os participantes de mercado; o estoque proporciona um colchão para o desbalanceamento entre a produção e consumo. Há cinco principais tipos de estoque:

- Material para entrega nos armazéns designados pela bolsa;

- Material para entrega na fábrica, no ponto de consumo, em trânsito ou em armazéns de distribuição;

- Material no mesmo estágio do ciclo de produção designado pela bolsa, mas que apresenta alguma diferença de especificação, tais como produto com teor de metal abaixo do padrão e que gera um prêmio menor; produto com teor de metal acima do especificado e que gera um prêmio maior; produto similar, mas não listado conforme especificação;

- Material em estágio anterior ao estágio do ciclo de produção designado pela bolsa;

- Material em estágio posterior ao estágio do ciclo de produção designado pela bolsa.

A London Metal Exchange (LME) é a principal bolsa de metais no mundo, incluindo alumínio, cobre, zinco, estanho, chumbo e níquel. Apesar da maioria dos contratos serem reservados para produtos primários, a LME também negocia contratos de ligas metálicas desde 1992. A LME opera sessões individuais para cada metal (rings), como também sessões para todos os metais (kerbs). Cada data de entrega é negociada individualmente e a liquidez concentra-se para contratos de preço à vista e de três meses.

No entanto, com relação à formação de preços, em termos de processos estocásticos, destacamse aplicações para metais industriais e preciosos, que são ativamente negociados em bolsas de metais. Esses metais incluem ouro, prata, platina, paládio, cobre, estanho, zinco, chumbo, níquel e alumínio. 
No tocante a estudos de modelagem econométrica da indústria do alumínio, um dos primeiros trabalhos foi o de Hojman (1981). O autor desenvolveu um modelo de regressão para a relação entre oferta, consumo e preços para mercado mundial de bauxita e alumínio a fim de avaliar o impacto de cartelização da indústria de 1967 a 1977. Os preços foram modelados como função da oferta, demanda e variação de estoques. Foi obtida correlação negativa de preços com variação de estoques. Os resultados não foram robustos pela limitação da base de dados e pela não realização do teste de causalidade de Granger.

Gilbert (1995) desenvolveu um modelo de equações simultâneas para preços, oferta, demanda e estoque para o mercado de alumínio com base em 26 observações anuais de 1966 a 1991. A previsão de preços foi superior apenas em relação a modelos simples empregados na indústria e para períodos até dois anos. Sow (1996) utilizou um modelo estrutural multivariado para fazer previsões de preços de alumínio, cobre, zinco e estanho até 2011. Também utilizou modelos de curva de aprendizado para desenvolver previsões de preços, mas não realizou análise comparativa dos modelos.

Ferretti (2003) avaliou a relação entre custos de produção e preço de alumínio, tendo como base observações anuais de 1982 a 1998. Argumentou que preços de componentes significativos de custo, como alumina e energia elétrica, a partir dos anos 80, passaram a ser atrelados a preços de alumínio como forma de mitigar riscos de mercado. Ao mesmo tempo, preços de aluminio passaram a ser estabelecidos pela London Metal Exchange (LME) a partir do inicío da década de 1980, e não por meio de modelos de custo mais margem. Ferretti concluiu que, no curto prazo, os preços de aluminio são determinantes dos custos. No longo prazo, Ferretti (2003) destaca que os custos de investimento em nova capacidade são os determinates principais de preços de alumínio.

Dooley e Lenihan (2005) analisaram a capacidade preditiva do modelo ARIMA e de um modelo simples baseado em curvas futuras para previsão de preços de zinco e chumbo, com vistas a melhor suportar o processo de planejamento de empresas do setor. Os resultados não foram conclusivos para zinco. Com relação ao chumbo, o modelo ARIMA proporcionou desempenho preditivo marginalmente superior em relação ao obtido por um modelo simples de curva futura usado como benchmark. 
Bernard et al. (2005) compararam a performance preditiva de preços de alumínio utilizando modelos ARCH, GARCH e modelos auto-regressivos em horizontes de tempo diário, semanal e mensal. Para dados de frequência diária e semanal, os modelos auto-regressivos apresentaram desempenho superior em relação aos modelos ARCH e GARCH. Com dados mensais, todos modelos apresentaram desempenho preditivo semelhante. No entanto, dependendo do período de tempo e de critério de avaliação das previsões, o modelo GARCH apresentou melhor desempenho.

No Brasil, Baltar (2009) desenvolveu previsão de preços de cobre utilizando modelo ARIMA. Foi analisada uma série histórica de retornos de preços de cobre com 19 anos de observações diárias entre 1990 e 2008, aplicando-se a metodologia tradicional de Box e Jenkins. O autor concluiu que o modelo proposto apresentou desempenho preditivo apenas razoável no curto prazo.

Ru e Ren (2012) avaliaram um modelo ARMA para previsão de preços de alumínio, tendo como período de estimação observações semanais de janeiro de 2006 a setembro de 2011. Foram projetados apenas quatro pontos fora da amostra, em setembro de 2011. Os resultados não foram robustos e não foi considerado no estudo comparação com outros modelos.

Geman e Smith (2012) testaram a teoria de estocagem utilizando seis metais comercializados pela LME, ou seja, alumínio, cobre, zinco, estanho, chumbo e níquel. A teoria da estocagem estabelece duas previsões envolvendo estoques de commodities. A primeira é que, em situações de escassez, ou quando os estoques estão em níveis baixos, o preço à vista é maior que preço futuro e que a volatilidade do preço à vista é maior que a volatilidade do preço futuro. Inversamente, em períodos em que não há escassez, as volatilidades do preço à vista e futuro são reduzidas. Incluindo dados da China, os resultados confirmaram a teoria de estocagem.

Mutafoglu et al. (2012) avaliaram a relação entre as posições futuras de traders para platina, ouro e prata e retornos de preços à vista de janeiro de 1993 a dezembro de 2009. Apesar de uma relação de causalidade ter sido encontrada entre posições de traders e retornos para períodos antes de quebras estruturais, o mesmo não foi confirmado para períodos após pontos de ruptura. A indicação é que as oscilações de retorno influenciam mais as posições de traders do que o contrário. 
Issler e Rodrigues (2012) desenvolveram análise comparativa de modelos para previsão do Metals Price Index (do Fundo Monetário Internacional), utilizando modelos AR, GARCH e VAR, bem como combinações de previsões e um modelo de correção de viés. A maioria dos modelos eram variações de modelos VAR. Foram utilizados critérios de informação tradicionais na comparação dos modelos. Os autores concluiram que combinar previsões é melhor para prever o Metals Price Index do que modelos individuais. Para previsões de um e três meses à frente, a combinação de média simples apresentou o melhor desempenho preditivo. De seis a quinze meses à frente, o modelo com correção de viés mostrou desempenho superior em relação aos demais.

Timmermann e Gargano (2012) utilizaram modelos de regressão com variáveis indepedentes macroeconômicas, tais como produção industrial, base monetária e taxas de juros, para avaliar o poder preditivo em termos de retorno de preço de commodities, incluindo metais. Concluiram que a capacidade preditiva do modelo era maior para matérias-primas industriais e metais e menor para alimentos e têxteis. A inflação não mostrou poder preditivo para previsões mensais, mas para horizontes trimestrais ou anuais. Os resultados sugeriram que a influência de inflação, base monetária e produção industrial é maior em períodos de recessão do que em crescimento.

\subsection{Modelos Individuais Utilizados na Previsão de Preços de Alumínio}

\subsubsection{SARIMA}

Como séries temporais econômicas normalmente apresentam um componente sazonal, como é o caso de preços de commodities, torna-se necessário incorporar o componente sazonal na abordagem ARIMA. Resulta assim a modelagem mais geral SARIMA, que inclui componentes sazonais autorregressivo SAR(P) e de média móvel SMA(Q), apresentado como SARIMA $(\mathrm{p}, \mathrm{d}, \mathrm{q}) \times(\mathrm{P}, \mathrm{D}, \mathrm{Q})$, onde D é a ordem de diferenciação sazonal.

Conforme Box, Jenkins e Reinsel (2008), onde B representa o operador de defasagem e $\left\{Z_{t}\right\} \sim N\left(0, \sigma^{2}\right)$ é um ruído branco, a forma geral do modelo SARIMA (p, d, q) x (P, D, Q)s para uma série $X_{t}$ é expressa por: 


$$
\begin{aligned}
& \left(1-\emptyset_{1} B^{1}-\ldots-\emptyset_{p} B^{p}\right)\left(1-\Phi_{1} B^{s}-\ldots-\Phi_{P} B^{s P}\right)\left(1-B^{d}\right)\left(1-B^{S}\right)^{D} X_{t}=\left(1+\theta_{1} B^{1}+\ldots+\right. \\
& \left.\theta_{q} B^{q}\right)\left(1+\Theta_{1} B^{s}+\ldots+\Theta_{Q} B^{s Q}\right) Z_{t}
\end{aligned}
$$

\subsubsection{SARIMAX}

O modelo SARIMAX pode ser expresso por um SARMAX, em que $\mathrm{X}_{\mathrm{t}}$ representa a série estacionária, incluindo um componente sazonal autoregressivo $\sum_{\mathrm{j}=1}^{\mathrm{P}} \Phi_{\mathrm{j}} \mathrm{X}_{\mathrm{t}-\mathrm{j}}$ de ordem $\mathrm{P}$ e período sazonal s e um componente sazonal de média móvel $\sum_{\mathrm{k}=1}^{\mathrm{Q}} \theta_{\mathrm{k}} \mathrm{Z}_{\mathrm{t}-\mathrm{k}}$ de ordem $\mathrm{Q}$ e período sazonal s. Conforme Stoffer e Shumway (2006), a forma geral do SARMAX é expresso por:

$$
\mathrm{X}_{\mathrm{t}}=\Gamma \mathrm{U}_{\mathrm{t}}+\sum_{\mathrm{j}=1}^{\mathrm{p}} \emptyset_{\mathrm{j}} \mathrm{X}_{\mathrm{t}-\mathrm{j}}+\sum_{\mathrm{j}=1}^{\mathrm{P}} \Phi_{\mathrm{j}} \mathrm{X}_{\mathrm{t}-\mathrm{j}}+\sum_{k=1}^{q} \theta_{\mathrm{k}} \mathrm{Z}_{\mathrm{t}-\mathrm{k}}+\sum_{\mathrm{k}=1}^{\mathrm{Q}} \Theta_{\mathrm{k}} \mathrm{Z}_{\mathrm{t}-\mathrm{k}}+\mathrm{Z}_{\mathrm{t}}
$$

em que:

$\Gamma$ : vetor $\mathrm{k} x \mathrm{r}$ dos coeficientes das variáveis exógenas;

$\mathrm{U}_{\mathrm{t}}$ : vetor $\mathrm{r}$ x 1 das variáveis exógenas para representar valores atuais e passados.

\subsubsection{Modelo Estrutural}

Formalmente proposto por Harvey (1989), o modelo estrutural modela uma série univariada em função de seus componentes não observáveis: tendência; sazonalidade; ciclo segundo uma abordagem linear. A denominação estrutural decorre do fato de se buscar uma modelagem por meio de componentes com uma clara interpretação da realidade.

O modelo geral linear aditivo é expresso por:

$\mathbf{X}_{\mathrm{t}}=\mu_{\mathrm{t}}+\gamma_{\mathrm{t}}+\omega_{\mathrm{t}}+\mathbf{Z}_{\mathrm{t}}$

em que $\mu_{\mathrm{t}}, \gamma_{\mathrm{t}}, \omega_{\mathrm{t}}$ são os componentes estocásticos de tendência, sazonalidade e ciclo e $\mathrm{Z}_{\mathrm{t}}$ é um ruído branco, conforme descritos na seção 2.2.1. Os componentes $\mu_{\mathrm{t}}, \gamma_{\mathrm{t}}, \omega_{\mathrm{t}}$ seguem as leis de formação abaixo. Os parâmetros a serem estimados são as variâncias $\sigma_{v}^{2}, \sigma_{\eta}^{2}, \sigma_{\varphi}^{2}, \sigma_{\psi}^{2}, \sigma_{\xi}^{2}$. O 
componente sazonal requer geralmente (s-1) equações de estado, onde s é o período sazonal; no caso abaixo, considerou-se $\mathrm{s}=4$.

(i) Tendência

$\mu_{\mathrm{t}}=\mu_{\mathrm{t}-1}+\beta_{\mathrm{t}}+v_{\mathrm{t}}, v_{\mathrm{t}} \sim N I\left(0, \sigma_{\mathrm{v}}^{2}\right)$

$\beta_{\mathrm{t}}=\beta_{\mathrm{t}-1}+\eta_{\mathrm{t}}, \eta_{\mathrm{t}} \sim N I\left(0, \sigma_{\eta}^{2}\right)$

(ii) Sazonalidade

$\gamma_{1 \mathrm{t}}=-\gamma_{1 \mathrm{t}-1}-\gamma_{2 \mathrm{t}}-\gamma_{3 \mathrm{t}}+\varphi_{\mathrm{t}}, \varphi_{\mathrm{t}} \sim N I\left(0, \sigma_{\varphi}^{2}\right)$

$\gamma_{2 \mathrm{t}}=\gamma_{2 \mathrm{t}-1}$

$\gamma_{3 \mathrm{t}}=\gamma_{3 \mathrm{t}-1}$

(iii) Ciclo

$\omega_{\mathrm{t}}=\lambda_{\mathrm{t}} \psi_{\mathrm{t}}, \psi_{\mathrm{t}} \sim N I\left(0, \sigma_{\psi}^{2}\right)$

$\psi_{\mathrm{t}}=\psi_{\mathrm{t}-1}+\xi_{\mathrm{t}}, \xi_{\mathrm{t}} \sim N I\left(0, \sigma_{\xi}^{2}\right)$

\subsection{Seleção de Modelos}

\subsubsection{Abordagem Tradicional}

Sejam $\left\{\hat{y}_{i t}\right\}_{t=1}^{T}$ e $\left\{\hat{y}_{j t}\right\}_{t=1}^{T}$ duas previsões de uma série temporal $\left\{y_{t}\right\}_{t=1}^{T}$ e os erros de previsão $\left\{\hat{e}_{i t}\right\}_{t=1}^{T}$ e $\left\{\hat{e}_{j t}\right\}_{t=1}^{T}$, em que $\hat{e}_{i t}=\left(y_{t}-\hat{y}_{i t}\right)$ e $\hat{e}_{j t}=\left(y_{t}-\hat{y}_{j t}\right)$.

As expressões (2.11) a (2.16) apresentam algumas medidas associadas aos erros de previsão:

- $\quad$ Erro Absoluto Médio (Mean Absolute Error - MAE)

$$
\mathrm{MAE}=\frac{\sum_{t=1}^{T}\left|\hat{e}_{i t}\right|}{T}
$$

- $\quad$ Erro Quadrático Médio (Mean Square Error - MSE) 
$\mathrm{MAE}=\frac{\sum_{t=1}^{T} \hat{e}_{i t}{ }^{2}}{T}$

- $\quad$ Raiz do Erro Quadrático Médio (Root Mean Square Error - RMSE)

$$
\mathrm{RMSE}=\sqrt{\mathrm{MSE}}
$$

- $\quad$ Média do Erro Absoluto Percentual (Mean Absolute Percentage Error - MAPE)

$$
\operatorname{MAPE}=\frac{\sum_{t=1}^{T}\left|\hat{e}_{i t} / y_{t}\right|}{T}
$$

- $\quad$ Raiz do Erro Quadrático Percentual (Mean Square Percentage Error - MSPE)

$$
\operatorname{MSPE}=\frac{\sum_{t=1}^{T}\left(\frac{\widehat{e}_{i t}}{y_{t}}\right)^{2}}{T}
$$

- Raiz do Erro Quadrático Médio Percentual (Root Mean Square Percentage Error - RMSPE)

$$
\mathrm{RMSPE}=\sqrt{\mathrm{MSPE}}
$$

As abordagens tradicionais para seleção do melhor modelo de previsão baseiam-se nas medidas apresentadas de (2.11) a (2.16) e, de modo geral, em alguma função relacionada aos erros de previsão. No entanto, tais medidas podem levar a conclusões errôneas decorrente do fato de existir apenas uma realização do processo estocástico. Um modelo pode ter um desempenho aparentemente superior a outro simplesmente por um resultado do acaso do que, de fato, por apresentar melhor poder preditivo.

A fragilidade da abordagem tradicional está associada ao problema de data snooping, que ocorre quando um conjunto de dados é usado mais de uma vez para fins de inferência e de seleção de modelos (WHITE, 2000). Tal fato leva à possibilidade de que um modelo possa ser escolhido não pela sua real acuracidade, porém mais pelo acaso na escolha dos dados. Data snooping é inevitável em análise de séries temporais pelo fato de se trabalhar com apenas uma 
série histórica para estimar o modelo de previsão. Apesar de ser um problema geral e reconhecido por pesquisadores, rotineiramente utilizam-se abordagens tradicionais. Leamer (1978, 1983) foi um dos primeiros a apontar tais problemas e indicar as fragilidades dos métodos tradicionais. Outros trabalhos relevantes incluem os de Mayer (1980), Miller (1981), Cox (1982), Lovell (1983). Lo e MacKinley (1990) chamaram a atenção deste fato de uma forma bem documentada e extensa.

Nenhum desses trabalhos, porém, resultou em um método rigoroso para avaliar se um modelo, de fato, apresentava melhor poder preditivo do que outros, levando em conta os efeitos de data snooping. Em 1995, Diebold e Mariano propuseram um método formal para solucionar este problema, mas considerando apenas dois conjuntos de modelos, o qual será discutido na seção 2.3.2.

\subsubsection{O Método de Diebold e Mariano}

Para cada período t e para um modelo i, é associada uma função de perda $\mathrm{g}\left(y_{t}, \hat{y}_{i t}\right)$, que, em geral, está relacionada diretamente ao erro de previsão, ou seja:

$\mathrm{g}\left(y_{t}, \hat{y}_{i t}\right)=\mathrm{g}\left(e_{i t}\right)$

A hipótese nula do método proposto por Diebold e Mariano (1995) é:

$H_{o}: \mathrm{E}\left[\mathrm{g}\left(e_{i t}\right)\right]=\mathrm{E}\left[\mathrm{g}\left(e_{j t}\right)\right]$,

ou seja, a função de perda dos dois modelos que geram as previsões $\hat{y}_{i t}$ e $\hat{y}_{j t}$ não é estatisticamente diferente.

Fazendo $d_{t}=\left[\mathrm{g}\left(e_{i t}\right)-\mathrm{g}\left(e_{j t}\right)\right]$, onde a $d_{t}$ é a perda diferencial amostral, a hipótese nula é reescrita como:

$H_{o}: \mathrm{E}\left[d_{t}\right]=0$ 
As hipóteses alternativas são dadas por duas situações:

$H_{a}: \mathrm{E}\left[d_{t}\right]>0$, o modelo $\mathrm{j}$ possui melhor poder preditivo que o modelo $\mathrm{i}$

$H_{a}: \mathrm{E}\left[d_{t}\right]<0$, o modelo i possui melhor poder preditivo que o modelo $\mathrm{j}$

Seja $\bar{d}=\frac{\sum_{t=1}^{T} d_{t}}{T}$ a perda média de previsão amostral. A estatística do teste de Diebold e Mariano (1995) é dada por:

$\mathrm{S}=\frac{\bar{d}}{\sqrt{\frac{2 \pi \overline{f_{d}}}{T}}}$

em que $\widehat{f_{d}}(0)$ é um estimador consistente de $f_{d}(0)=\frac{1}{2 \pi} \sum_{\tau=-\infty}^{\infty} \gamma_{d}(\tau)$ é a densidade espectral de $\bar{d}$. Sob hipótese nula e assintoticamente, Dielbold e Mariano (1995) demonstram que:

$\mathrm{S} \sim \mathrm{N}(0,1)$

Portanto, utilizando testes usuais da distribuição normal padrão, pode-se determinar qual o melhor modelo de previsão.

\subsubsection{Model Confidence Set (MCS)}

Diferentemente dos modelos convecionais, o Model Confidence Set (MCS), proposto por Hansen, Lunde e Nason (2011), é capaz de fazer comparações múltiplas de modelos com base em um dado nível descritivo, levando em conta as limitações dos dados. Tal fato possibilita a seleção de um conjunto de modelos que apresentam acuracidade de previsão equiparável, a um dado nível descritivo definido pelo MCS.

O objetivo do Model Confidence Set (MCS) é determinar o conjunto de modelos $M^{*}$, que contenha o(s) melhor(es) modelo(s) de uma coleção de modelos $M^{0}$, em que o conceito de "melhor" é definido a partir de um critério associado à performance de previsão. O MCS estima 
um conjunto $\widehat{M^{*}}$, que é o conjunto que contém os melhores modelos para um dado nível descritivo.

Uma contribuição importante do MCS é que esta abordagem reconhece as limitações dos dados. Dados com mesma capacidade informacional resultam em um $\widehat{M^{*}}$ que contém apenas um modelo. Dados com menor capacidade informacional resultam em um conjunto de modelos com performance de previsão equiparáveis, a um dado nível descritivo do MCS. Portanto, o MCS difere dos métodos de seleção de modelos convencionais, que determinam apenas um modelo como o melhor entre todos os ajustados, com base em apenas um critério, independentemente da natureza dos dados.

O método de seleção pelo MCS é baseado em um teste de equivalência, $\delta_{M}$ e uma regra de eliminação $e_{M}$. O teste de equivalência é aplicado para o conjunto $M=M^{0}$. Se $\delta_{M}$ é rejeitado, então há evidência de que os modelos não apresentam o mesmo poder preditivo e $e_{M}$ é utilizada para eliminar os modelos com baixa performance de previsão. Esse procedimento é repetido até que $\delta_{M}$ é aceito e $\widehat{M^{*}}$ é definido para um conjunto dos melhores modelos. Utilizando um nível descritivo $\alpha$ em todos os testes, o método assegura que $\lim _{n \rightarrow \infty}\left(M^{*} \widehat{C}_{M^{*}}{ }_{1-\alpha}\right) \geq 1-\alpha$. Quando $\widehat{M^{*}}$ contém apenas um modelo, tem-se o caso forte em que $\lim _{n \rightarrow \infty}\left(M^{*}=\widehat{M}^{*}{ }_{1-\propto}\right)$ $=1$.

O MCS também gera níveis descritivos para cada modelo comparado. Para um dado modelo i $\in M^{0}$, o nível descritivo $\widehat{p_{l}}$ é o threshold para o qual $\mathrm{i} \in \widehat{M}^{*}{ }_{1-\alpha}$, se e somente se $\widehat{p_{l}}>\alpha$. Dessa forma, um modelo com baixo nível descritivo é improvável de estar entre os melhores de acuracidade de previsão.

O algoritmo do MCS baseia-se nos seguintes passos: (i) inicialmente, fazer $M=M^{0}$. Testar a hipótese $H_{0, M}$ usando $\delta_{M}$ ao nível descritivo $\alpha$. Se $H_{0, M}$ é aceita, então define-se $\widehat{M}^{*}{ }_{1-\alpha}=M$, caso contrário, usa-se $e_{M}$ para eliminar o modelo de baixa performance e repete-se a rotina. 


\section{COMBINAÇÃO DE PREVISÕES}

\subsection{Histórico}

Várias e diferentes previsões de uma mesma variável podem ser disponíveis para tomada de decisões em setores públicos ou privados dependendo dos modelos empregados. O tomador de decisão deseja utilizar o modelo com previsões de desempenho superior. Nesse contexto, coloca-se a questão: um método de combinação das previsões individuais pode gerar novas previsões com acuracidade superior?

A primeira abordagem teórica para combinar previsões foi desenvolvida por Bates e Granger (1969), com base no conceito de ganhos de diversificação de um portólio. O modelo de Bates e Granger (1969) supõe que o usuário das diferentes previsões individuais não tem acesso à informação que baseia os modelos. Por exemplo, suponha que estamos interessados em projetar uma variável y, e que duas previsões, $\hat{y}_{1}$ e $\hat{y}_{2}$, da sua média condicional são disponíveis. Seja a primeira previsão baseada nas variáveis $\hat{x}_{1}$ e $\hat{x}_{2}$, ou seja, $\hat{y}_{1}=\mathrm{g}_{1}\left(\hat{x}_{1} \mathrm{e} \hat{x}_{2}\right)$, enquanto a segunda previsão é baseada nas variáveis $\hat{x}_{3}$ e $\hat{x}_{4}$, ou seja, $\hat{y}_{2}=\mathrm{g}_{2}\left(\hat{x}_{3}\right.$ e $\left.\hat{x}_{4}\right)$. Assumindo que todas as variáveis independentes tenham pesos diferentes de zero nas previsões e que as variáveis $\mathrm{x}$ sejam observáveis, poderia ser criada uma terceira previsão $\hat{y}_{1}=\mathrm{g}_{3}\left(\hat{x}_{1}, \hat{x}_{2}, \hat{x}_{3}, \hat{x}_{4}\right)$. Por outro lado, se apenas as previsões $\hat{y}_{1}$ e $\hat{y}_{2}$ são observadas pelo usuário das projeções (mas não as variáveis x), então a única opção seria fazer a combinação das previsões, ou seja, desenvolver um modelo onde $\hat{y}_{\mathrm{c}}=\mathrm{g}_{\mathrm{c}}\left(\hat{y}_{1}, \hat{y}_{2}\right)$.

De acordo com Bates e Granger (1969), o problema é determinar os pesos das previsões individuais que geram um modelo combinado com acuracidade de previsão superior. O modelo de Bates e Granger (1969) assume que as variâncias dos erros de duas previsões, por exemplo, $\sigma_{1}$ e $\sigma_{2}$, sejam constantes ao longo do tempo. Também assume-se que ambas previsões não são enviesadas. A previsão combinada é obtida pela combinação linear das previsões individuais, sendo k o peso da primeira previsão e (1-k) o peso da segunda previsão. A variância dos erros da previsão combinada é dada por (3.1):

$\sigma_{c}^{2}=k^{2} \sigma_{1}^{2}+(1-k)^{2} \sigma_{2}^{2}+2 k \rho \sigma_{1}(1-k) \sigma_{2}$ 
em que:

k é o peso dado à primeira previsão

(1-k) é o peso dado à segunda previsão

$\rho$ é o coeficiente de correlação entre os erros da primeira e segunda previsões

A escolha do peso $\mathrm{k}$ deve ser feita tal que os erros da combinação sejam minimizados. Especificamente, o objetivo é minimizar a variância dos erros da combinação $\sigma_{c}^{2}$. Diferenciando (3.1) em relação a $\mathrm{k}$ e igualando a zero, obtem-se a variância mínima da combinação, $\sigma_{c}^{2}$, quando se tem $\mathrm{k}$ dado por (3.2):

$k=\frac{\sigma_{2}^{2}-\rho \sigma_{1} \sigma_{2}}{\sigma_{1}^{2}+\sigma_{2}^{2}-2 \rho \sigma_{1} \sigma_{2}}$

Quando $\rho=0,(3.2)$ se reduz a:

$k=\frac{\sigma_{2}^{2}}{\sigma_{1}^{2}+\sigma_{2}^{2}}$

Se o peso k é determinado por (3.2), então o valor de $\sigma_{c}^{2}$ não é maior que a menor das duas variâncias individuais. O valor ótimo de k não é conhecido a priori quando se faz a combinação das previsões. $\mathrm{O}$ valor de $\mathrm{k}$ pode mudar em função do desempenho relativo da acuracidade das previsões individuais.

Portanto, conforme Bates e Granger (1969), a combinação das previsões $C_{T}$ é dada por (3.4):

$C_{T}=k_{T} f_{1, T}+\left(1-k_{T}\right) f_{2, T}$

em que:

T é o período de tempo da previsão

$f_{1, T}$ é a primeira previsão em $\mathrm{T}$

$f_{1, T}$ é a segunda previsão em T 
Os autores propuseram cinco métodos de estimação de pesos a partir de duas previsões individuais. Para cada método, $\mathrm{k}_{\mathrm{T}}$ foi o peso atribuído à primeira série e $\left(1-\mathrm{k}_{\mathrm{T}}\right)$, o peso atribuído à segunda série. Os pesos $\mathrm{k}_{\mathrm{T}}$ em todos os cinco casos foram determinados com base nos erros passados das séries individuais, exceto $\mathrm{k}_{1}$, a que os autores atribuiram 0,5.

O conceito utilizado Bates e Granger (1969) foi reforçado por Nelson (1972), através de um desenvolvimento relativamente independente, com aplicação na economia norte-americana. Newbold e Granger (1974) mantiveram todas as suposições e os métodos de combinação de Bates e Granger (1969), e aumentaram o número de previsões individuais com base em modelos ARIMA, Holt-Winters e de autoregressão stepwise. Newbold e Granger (1974) concluiram que o desempenho preditivo da combinação dos três modelos foi superior ao dos modelos individuais.

Makridakis et al. (1982; 1983) mostraram que um modelo de média aritmética simples e outro de média ponderada apresentaram melhor desempenho preditivo entre 16 modelos individuais, além de 4 outros métodos de combinações de pesos.

Makridakis e Winkler (1983) analisaram a acuracidade de combinações de previsões por meio de 5 métodos de média ponderada a partir de 10 modelos individuais. Os principais modelos individuais utilizados foram: "naive" (autoregressivo); média móvel simples; alisamento exponencial simples; alisamento exponencial com resposta adaptativa; alisamento exponencial linear de Holt; alisamento exponencial de Brown; alisamento exponencial quadrático de Brown; regressão linear; método de Holt-Winter. Os dois métodos que apresentaram acuracidade superior foram a ponderação por meio do inverso da soma dos erros quadrados. $\mathrm{O}$ segundo foi baseado no alisamento exponencial dos pesos obtidos no primeiro método. Os autores destacaram que a acuracidade da combinação depende de quão distintos são os modelos individuais, mas o desempenho da projeção combinada alcança uma saturação com quatro ou cinco previsões individuais. As conclusões de Makridakis e Winkler (1983) confirmaram os resultados de Newbold e Granger (1974).

Um uso mais efetivo da informação pode ser obtido pela variação dos pesos ao longo do tempo. Uma regressão com parâmetros variantes no tempo, por meio de um modelo estrutural e filtro de Kalman é possível para tal fim. Um método diferente foi proposto por Engle et al. (1984). Nesse trabalho, os métodos tradicionais de pesos foram utilizados nas combinações de duas 
previsões de inflação, com base nas variâncias e covariâncias dos erros, mas as variâncias desenvolvidas foram modeladas condicionalmente aos valores passados por meio do método ARCH. Apesar de ser potencialmente útil, esse método se mostrou mais complexo e menos atrativo que as técnicas de combinações usuais para minimização de erros.

Quando há muitas previsões individuais, a complexidade da aplicação dos métodos de combinação pode aumentar. Uma maneira pragmática de reduzir complexidade consiste em fazer uma seleção dos modelos individuais, descartando a maioria, e deixando um conjunto com poucos e melhores modelos. Figlewski (1983) propôs uma abordagem interessante nessa linha, usando dados de pesquisas de expectativa inflação. A previsão combinada no trabalho Figlewski (1983) foi superior que a de uma média simples.

Granger e Ramanathan (1984) destacaram que os métodos convencionais de combinação linear de previsões poderiam ser vistos como uma forma estruturada de regressão. Concluiram que o método de combinação linear é equivalente ao método de mínimos quadrados ordinários, assumindo a previsão combinada como variável dependente e as previsões individuais como variáveis independentes.

Granger e Newbold (1986) argumentaram que as previsões individuais são combinadas de forma linear usando algum mecanismo de estimação dos pesos, não necessariamente positivos ou normalizados (ou seja, a soma dos pesos é 1), com o objetivo de minimizar a variância dos resíduos. O modelo de combinação geral linear, h passos à frente é escrito por (3.5):

$\hat{y}_{C L, T+h}=\sum_{j=1}^{k} \widehat{w}_{j t} \hat{y}_{j, T+h}$

em que:

$\hat{y}_{C L, T+h}$ é a previsão combinada no instante $\mathrm{T}+\mathrm{h}$

$\widehat{w}_{j t}$ é o peso estimado em $\mathrm{T}+\mathrm{h}$ para o modelo $\mathrm{j}, \hat{y}_{j t}$, assumindo a amostra $\left(\hat{y}_{1}, \ldots, \hat{y}_{T}\right)$

$\hat{y}_{j, T+h}$ é a previsão do modelo j em $\mathrm{T}+\mathrm{h}$

Além do conceito de diversificação de ganhos de portfolio, uma segunda razão para o uso de combinação de previsões é que previsões individuais podem ser afetadas diferentemente por 
choques estruturais. Tal fato foi destacado por Winkler (1989), Hendry e Clements (2002) e Alfioli e Timmerman (2006).

Alguns modelos podem se adaptar rapidamente e são apenas temporariamente afetados por choques, enquanto outros têm parâmetros que se ajustam lentamente quando afetados por choques estruturais. Quanto mais dados são disponíveis e mais distantes após o choque estrutural mais recente, espera-se que modelos com adaptação lenta apresentem melhor acuracidade que modelos com rápida resposta ao choque, uma vez que os parâmetros daqueles são estimados com maior precisão. Por outro lado, quanto mais dados são disponíveis e menos distantes após o choque estrutural mais recente, os modelos com rápida resposta ao choque apresentam melhor desempenho que os de adaptação lenta. Dessa forma, combinações de previsões a partir de modelos com diferentes graus de resposta a choques estruturais devem apresentar melhor desempenho preditivo que modelos individuais. Esse resultado foi confirmado por Pesaran e Timmermann (2005).

Uma terceira razão para o uso de combinações de previsões é que modelos individuais podem estar sujeitos a problemas de viés de especificação, o que foi destacado por Makridakis (1989) e Stock e Watson $(2001,2004)$. Mesmo assumindo estacionariedade, o real processso gerador tende a ser bem mais complexo e de maior dimensão que o assumido pelo modelo mais geral e flexível disponível. Dessa forma, considerando modelos de previsão como aproximações locais, é pouco provável que o mesmo modelo individual domine todos os demais em todos os instantes de tempo. Combinar projeções utilizando diferentes modelos pode ser visto como uma forma de tornar a previsão mais robusta contra vieses de especificações e erros de medida embutidos nas previsões individuais. Stock e Watson (2004) também argumentaram que nem sempre métodos mais sofisticados e complexos de combinação apresentam melhor acuracidade que métodos mais simples. Makridakis e Winker (1983) defenderam a utilização da combinação por meio de uma média simples das previsões a empregar um modelo mais complexo, sem ganhos significativos de acuracidade. Taylor e Bunn (1998) destacaram que a média simples talvez fosse o método de combinação mais utilizado pelo fato de proporcionar uma compensação importante entre as previsões individuais.

Diebold e Lopez (1996) propuseram que a seleção dos modelos de previsão seja determinada através da minimização de uma função de perda, que é uma função associada ao desvio entre o valor observado e o previsto da variável dependente, fora da amostra. A função de perda $\mathrm{L}$ 
(loss) associada a uma variável dependente y está associada ao erro de previsão $e_{t+h}$, conforme (3.6):

$\mathrm{L}=\mathrm{L}\left(e_{t+h}\right)$,

$e_{t+h}=y_{t+h}-\hat{y}_{t+h}$

Nesse contexto, um quarto argumento para o uso de combinação de previsões está relacionado ao fato de que as escolhas dos modelos individuais podem ter sido baseados em diferentes funções de perda. Suponha, por exemplo, que uma previsão A está sub-estimada e uma previsão B está sobre-estimada. Se o viés é constante ao longo do tempo, a simples inclusão de uma constante na previsão combinada retira esse efeito indesejável. Evans (2003) destacou a possibilidade do uso de um modelo de regressão para combinação de previsões com uma constante aditiva.

Christoffersen e Diebold (1997) investigaram, no entanto, o caso em que o viés é proporcional à variância condicional da variável dependente. Assumindo que as previsões tenham volatilidade similar, os autores argumentaram que, se for usada uma função de perda mais simétrica do que aquelas utilizadas na construção das previsões individuais, é possível obter uma combinação com maior desempenho preditivo que as previsões individuais.

Hendry e Clements (2002) argumentaram que combinações de previsões podem fornecer resultados empíricos favoráveis porque fornecem uma espécide de seguro contra externalidades e quebras estruturais. Os autores desenvolveram várias simulações envolvendo quebras estruturais e concluiram que combinações de previsões proporcionam maior acuracidade de previsão por meio de uma translação do intercepto da variável relacionada ao processo gerador de dados.

Stock e Watson (2006) analisaram o caso em que o número de previsões individuais é grande. Os autores argumentaram que o uso de combinação de previsões explora melhor o uso de uma base de informação mais rica e proporciona maior robustez contra instabilidade estrutural dos modelos individuais. Destacaram vários métodos de combinações, tais como combinação simples, erro quadrático médio invertido e parâmetros variantes no tempo. 
Trabalhos subsequentes abordaram diversos tópicos, tais com os de Lam et al. (2001), com relação a abordagens para minimização de erros; Chan et al. (2004), sobre utilização de pesos variáveis; Eliott e Timmermman (2005), relativo ao uso de modelos de mudança de regime; Liang et al. (2006), no tocante a modelos de combinação linear; Fan e Deng (2007), na utilização de erros de previsão para construir pesos variáveis; Kim (2008), sobre modelos de auto-regressão generalizada; Smith e Wallis (2009), sobre erro de amostras finitas na estimativa do peso combinado.

Issler e Lima (2009) propuseram uma nova abordagem para combinação de previsões, usando correção de viés, que se mostrou potencialmente superior a outras técnicas em um contexto assintótico. Kolassa (2011) destacou a construção de pesos com base no critério de informação de Akaike (AIC). Bruggemman e Luktepohl (2013) demonstraram que a previsão combinada, com base na minimização do erro quadrático médio, apresentou melhor acuracidade utilizando dados agregados do PIB e da base monetária dos Estados Unidos do que utilizando dados desagregados. Hsiao e Wan (2014) compararam combinações utilizando ponderação pelo AIC, regressão, método bayesiano e minimização de erros quadráticos.

Tópicos recentes e para pesquisas futuras abordam aspectos tais como combinação de densidades de probabilidade, pesos variantes no tempo e combinações com funções de perda assimétricas.

\subsection{O Problema de Combinação de Previsões}

Considere o problema de projetar no instante t o valor futuro de uma variável dependente, y, após h períodos, cuja realização é denotada por $y_{t+h}$. Um conjunto de variáveis independentes x também poderia ser incluído no modelo, sem perda de generalização. Refere-se t como o instante de previsão e h como o horizonre de previsão.

A informação conhecida no tempo $t$ será denotada por $\mathrm{J}_{\mathrm{t}}$, que inclui um vetor $\widehat{\boldsymbol{y}}_{t+h, t}$ de $\mathrm{N}$ previsões individuais dadas por (3.8):

$\widehat{\boldsymbol{y}}_{t+h, t}=\left(\hat{y}_{t+h, t, 1}, \hat{y}_{t+h, t, 2}, \ldots, \hat{y}_{t+h, t, N}\right)^{\prime}$ 
$\mathrm{J}_{\mathrm{t}}$ inclui os valores projetados de $\mathrm{t}$ até $\mathrm{t}+\mathrm{h}$, bem como os valores observados de cada previsão individual até t, ou seja:

$\mathrm{f}_{t}=\left(\widehat{\boldsymbol{y}}_{t+1, t}, \widehat{\boldsymbol{y}}_{t+2, t}, \ldots, \widehat{\boldsymbol{y}}_{t+h, t}, y_{1}, \ldots, y_{t}\right)$

Seja $\hat{y}_{t+h, t}^{c}=C\left(\hat{\boldsymbol{y}}_{t+h, t} ; \boldsymbol{w}_{t+h, t}\right)$ a combinação de previsões pontuais em que em $\mathrm{t}+\mathrm{h}$, dependente das previsões individuais $\widehat{\boldsymbol{y}}_{t+h, t}$, bem como do vetor de pesos $\boldsymbol{w}_{t+h, t} \in \mathrm{W}_{\mathrm{t}}, \mathrm{W}_{\mathrm{t}}$ é geralmente assumido como um subconjunto de $\mathbb{R}^{N}$ e $w_{t+h, t}$ pode ser variante no tempo. Dessa forma, por exemplo, pesos iguais resultariam em:

$g\left(\widehat{\boldsymbol{y}}_{t+h, t} ; \boldsymbol{w}_{t+h, t}\right)=(1 / \mathrm{N}) \sum_{j=1}^{N} \hat{y}_{t+h, t, j}$

Assume-se que a função de perda $\mathrm{L}$ depende apenas dos erros de previsão da combinação, o que é assumido na grande maioria dos estudos em combinações de previsões, conforme (3.9):

$\mathrm{L}=\mathrm{L}\left(e_{t+h, t}\right)$

$e_{t+h, t}^{c}=y_{t+h, t}-g\left(\hat{\boldsymbol{y}}_{t+h, t} ; \boldsymbol{w}_{t+h, t}\right)$

Os pesos da combinação ótima, $\boldsymbol{w}_{t+h, t}^{*}$ minimizam a esperança condicional da função de perda $\mathrm{L}\left(e_{t+h, t}^{c} \mid\left(\boldsymbol{w}_{t+h, t}\right)\right)$, dada a informação passada $\mathrm{f}_{t}$, conforme (3.10):

$\boldsymbol{w}_{t+h, t}^{*}=\min \mathrm{E}\left[\mathrm{L}\left(e_{t+h, t}^{c} \mid\left(\boldsymbol{w}_{t+h, t}\right)\right) \mid \mathrm{f}_{t}\right]$

Normalmente, assume-se que a função de perda é baseada no erro quadrático médio (EQM)

$\mathrm{L}\left(y_{t+h}, \hat{y}_{t+h, t}\right)=L\left(y_{t+h}-\hat{y}_{t+h, t}\right)^{2}$

Nesse caso, procura-se minimizar o EQM, a fim de determinar a combinação $C\left(\widehat{\boldsymbol{y}}_{t+h, t} ; \boldsymbol{w}_{t+h, t}\right)$ que melhor se aproxima da esperança condicional, $\mathrm{E}\left[y_{t+h} \mid \widehat{\boldsymbol{y}}_{t+h, t}\right]$. 
Dois passos principais envolvem a determinação da combinação de previsões. O primeiro consiste na determinação das previsões pontuais $\widehat{\boldsymbol{y}}_{t+h, t}$. A única diferença da abordagem usual utilizada em modelos de previsão é que as variáveis independentes são as previsões de modelos individuais.

O segundo passo agrega o vetor $\widehat{\boldsymbol{y}}_{t+h, t}$ na combinação $C\left(\widehat{\boldsymbol{y}}_{t+h, t} ; \boldsymbol{w}_{t+h, t}\right)$. O segundo passo normalmente gera um modelo mais simples e parcimonioso em relação a um modelo individual mais geral e flexível com base nas variáveis independentes disponíveis. Em geral, é de se esperar que a agregação de informação aumente o viés das projeções combinadas, mas, ao mesmo tempo, reduz-e a variância dos erros da combinação. $\mathrm{Na}$ medida do possível, a combinação desejada deve buscar uma otimização entre esses dois componentes. Isso se torna particularmente claro quando se usa o EQM na função de perda, onde a função objetiva é igual ao erro ao quadrado mais a variância dos erros das previsões:

$\mathrm{E}\left[e_{t+h, t}^{2}\right]=\mathrm{E}\left[e_{t+h, t}\right]^{2}+\operatorname{Var}\left(e_{t+h, t}\right)^{2}$

\subsection{Combinação de Previsões com Função de Perda dada pelo Erro Quadrático Médio}

Considerando o caso de combinações lineares, em que se deseja determinar os pesos dados por (3.15):

$\boldsymbol{w}_{t+h, t}=\left(w_{t+h, t, 1}, \ldots, w_{t+h, t, N}\right)^{\prime}$

A combinação resultante será denotada por (3.16):

$\hat{y}_{t+h}^{c}=\widehat{\boldsymbol{w}}_{t+h}^{\prime} \widehat{\boldsymbol{y}}_{t+h}$

Segundo Timmermann (2006), considerando como função de perda o erro quadrático médio $(\mathrm{EQM})$, eminimizando $\mathrm{E}\left[e_{t+h, t}^{2}\right]=\mathrm{E}\left[\left(y_{t+h}-\widehat{\boldsymbol{w}}_{t+h}^{\prime} \widehat{\boldsymbol{y}}_{t+h}\right)^{2}\right]$, tem-se: 


$$
\begin{array}{r}
\boldsymbol{w}_{t+h, t}^{*}=\min \left(\left(\mu_{y t+h, t}-\widehat{\boldsymbol{w}}_{t+h, t}^{\prime} \widehat{\boldsymbol{\mu}}_{\hat{\boldsymbol{y}} t+h, t}\right)^{2}+\sigma_{y t+h, t}^{2}+\right. \\
\left.+\widehat{\boldsymbol{w}}_{t+h, t}^{\prime} \sum_{\hat{\boldsymbol{y}} \hat{\boldsymbol{y}} t+h, t} \widehat{\boldsymbol{w}}_{t+h, t}-2 \widehat{\boldsymbol{w}}_{t+h, t}^{\prime} \sigma_{y \hat{\boldsymbol{y}} t+h, t}\right)
\end{array}
$$

De (3.17), obtem-se a condição de primeira ordem:

$\frac{\partial E\left[e_{t+h, t}^{2}\right]}{\partial \boldsymbol{w}_{t+h, t}}=-\left(\mu_{y t+h, t}-\widehat{\boldsymbol{w}}_{t+h, t}^{\prime} \widehat{\boldsymbol{\mu}}_{\widehat{\boldsymbol{y}} t+h, t}\right) \widehat{\boldsymbol{\mu}}_{\widehat{\boldsymbol{y}} t+h, t}+\sum_{\widehat{\boldsymbol{y}} \hat{y} t+h, t} \widehat{\boldsymbol{w}}_{t+h, t}-\sigma_{y \widehat{y} t+h, t}=0$

Assumindo que $\sum_{\widehat{y} \widehat{y} t+h, t}$ é inversível, tem-se a solução:

$\boldsymbol{w}_{t+h, t}^{*}=\left(\boldsymbol{\mu}_{\widehat{y} t+h, t} \boldsymbol{\mu}_{\hat{\boldsymbol{y}} t+h, t+}^{\prime} \sum_{\hat{y} \hat{y} t+h, t}\right)^{-1}-\left(\boldsymbol{\mu}_{\widehat{y} t+h, t} \mu_{y t+h, t}+\boldsymbol{\sigma}_{y \widehat{y} t+h, t}\right)$

A solução é ótima na população quando $y_{t+h}$ e $\widehat{\boldsymbol{y}}_{t+h, t}$ são distribuições normais conjuntas, pois a esperança condicional $\mathrm{E}\left[y_{t+h} \mid \mathrm{e} \widehat{\boldsymbol{y}}_{t+h, t}\right]$ será linear em $\widehat{\boldsymbol{y}}_{t+h, t}$. Uma constante pode ser incluida na solução, uma alternativa sugerida por Granger e Ramanathan (1984) e por Eliott e Timmerman (2004). Em geral, os pesos tendem a ser maiores para previsões mais acuradas e a constante corrige viés nas previsões.

\subsection{Ganhos de Diversificação}

A primeira abordagem teórica para combinar previsões, conforme Bates \& Granger (1969), baseia-se no conceito de ganhos de diversificação de um portólio, o que foi também argumentado por Winkler (1989). Para esse fim, seja uma combinação de duas previsões, que geram dois erros:

$$
\begin{aligned}
& e_{1}=y-\hat{y}_{1} \\
& e_{2}=y-\hat{y}_{2}
\end{aligned}
$$

Por simplicação de notação, foram excluidos os subscriptos de tempo. Assumindo que as previsões não sejam enviesadas, tem-se:

$e_{1} \sim N\left(0, \sigma_{1}^{2}\right), \sigma_{1}^{2}=\operatorname{Var}\left(e_{1}\right)$ 
$e_{2} \sim N\left(0, \sigma_{2}^{2}\right), \sigma_{2}^{2}=\operatorname{Var}\left(e_{2}\right)$

$\sigma_{12}=\rho_{12} \sigma_{1} \sigma_{2}$

A covariância entre $e_{1}$ e $e_{2}$ é $\sigma_{12}$ e a correlação entre $e_{1}$ e $e_{2}$ é $\rho_{12}$. Considere-se que a soma dos pesos seja um, com pesos w e (1-w) para a primeira e segunda previsões, respectivamente. O erro de previsão da combinação será:

$e^{c}=\mathrm{w} e_{1}+(1-w) e_{2}$

Por construção, a média é zero e a variância será:

$\sigma_{c}^{2}(w)=w^{2} \sigma_{1}^{2}+(1-\mathrm{w})^{2} \sigma_{2}^{2}+2 \mathrm{w}(1-\mathrm{w}) \sigma_{12}$

Diferenciando a expressão (3.29) com relação a w e resolvendo para a condição de primeira ordem, tem-se:

$W^{*}=\frac{\sigma_{2}^{2}-\sigma_{12}}{\sigma_{1}^{2}+\sigma_{2}^{2}-2 \sigma_{12}}$

$1-w^{*}=\frac{\sigma_{1}^{2}-\sigma_{12}}{\sigma_{1}^{2}+\sigma_{2}^{2}-2 \sigma_{12}}$

Um peso maior é atribuído a modelos que geram previsões mais acuradas (com menor variância do erro de previsão). Quando $\rho_{12}>\sigma_{2} / \sigma_{1}$, a combinação dos pesos não é convexa e um dos pesos será maior que um, e outro será negativo, conforme Bunn (1985).

Inserindo $\mathrm{w}^{*}$ na função objetiva, tem-se a variância do erros da combinação associada aos pesos ótimos:

$\sigma_{c}^{2}\left(w^{*}\right)=\frac{\sigma_{1}^{2} \sigma_{2}^{2}\left(1-\rho_{12}^{2}\right)}{\sigma_{1}^{2}+\sigma_{2}^{2}-2 \rho_{12} \sigma_{1} \sigma_{2}}$

Pode ser verificado que $\sigma_{c}^{2}\left(w^{*}\right)<\min \left(\sigma_{1}^{2}, \sigma_{2}^{2}\right)$. O ganho de diversificação será zero em três casos particulares: 
(i) $\sigma_{1}=\sigma_{2}=0$

(ii) $\sigma_{1}=\sigma_{2}$

(iii) $\rho_{12}=\sigma_{2} / \sigma_{1}$

Vale ressaltar a comparação entre a variância do erro da combinação ótima e àquela associada a uma combinação em que os pesos são inversamente proporcionais ao erro quadrático médio e que, portanto, ignora a correlação entre os erros de previsão:

$w_{i n v}=\frac{\sigma_{2}^{2}}{\sigma_{1}^{2}+\sigma_{2}^{2}}$

$1-w_{i n v}=\frac{\sigma_{1}^{2}}{\sigma_{1}^{2}+\sigma_{2}^{2}}$

Esses pesos geram a variância do erro de previsão:

$\sigma_{i n v}^{2}=\frac{\sigma_{1}^{2} \sigma_{2}^{2}\left(\sigma_{1}^{2}+\sigma_{2}^{2}+2 \rho_{12} \sigma_{1} \sigma_{2}\right)}{\left(\sigma_{1}^{2}+\sigma_{2}^{2}\right)^{2}}$

Determina-se que a razão entre a variância do erro de previsão com os pesos associados ao inverso do erro quadrático médio sobre aquela com os pesos ótimos é:

$\frac{\sigma_{i n v}^{2}}{\sigma_{c}^{2}\left(w^{*}\right)}=\left(\frac{1}{1-\rho_{12}^{2}}\right)\left(1-\left(\frac{2 \sigma_{12}}{\sigma_{1}^{2}+\sigma_{2}^{2}}\right)^{2}\right)$

Se $\sigma_{1} \neq \sigma_{2}$, a razão (3.36) é maior que um, exceto quando $\rho_{12}=0$. Quando $\sigma_{1}=\sigma_{2}$, a razão é igual a um, não importando o valor de $\rho_{12}$; nesse caso, $\sigma_{i n v}^{2}=\sigma_{c}^{2}\left(w^{*}\right)=0,5$. Pesos iguais são ótimos quando as duas variâncias dos erros de previsão são iguais, não importando a correlação entre eles.

Tem-se outro benchmark interessante quando os pesos são iguais, ou seja:

$\hat{y}^{e w}=(1 / 2)\left(\hat{y}_{1}+\hat{y}_{2}\right)$

Nesse caso, a variância do erro da previsão é: 
$\sigma_{e w}^{2}=\frac{1}{4} \sigma_{1}^{2}+\frac{1}{4} \sigma_{2}^{2}+\frac{1}{2} \sigma_{1} \sigma_{2} \rho_{12}$

A razão $\sigma_{e w}^{2} / \sigma_{c}^{2}\left(\mathrm{w}^{*}\right)$ será:

$\frac{\sigma_{i n v}^{2}}{\sigma_{c}^{2}\left(w^{*}\right)}=\frac{\left(\sigma_{1}^{2}+\sigma_{2}^{2}\right)-4 \sigma_{12}^{2}}{4 \sigma_{1}^{2} \sigma_{2}^{2}\left(1-\rho_{12}^{2}\right)}$

Essa razão é maior que um, exceto quando $\sigma_{1}=\sigma_{2}$.

Por fim, como medida geral dos ganhos de diversificação, comparemos $\sigma_{c}^{2}\left(\mathrm{w}^{*}\right)$ com $\min \left(\sigma_{1}^{2}, \sigma_{2}^{2}\right)$. Supondo $\sigma_{1}>\sigma_{2}$ e seja $\mathrm{k}=\sigma_{2} / \sigma_{1}$. Tem-se a razão:

$\frac{\sigma_{c}^{2}\left(w^{*}\right)}{\sigma_{2}^{2}}=\frac{1-\rho_{12}^{2}}{1+k^{2}-2 \rho_{12} k}$

O ganho de diversificação, medido por essa razão, é uma função que depende da correçação entre as duas previsões, $\rho_{12}$, e da razão k, entre a variância dos erros de previsão. A derivada o ganho de diversificação em relação a $\rho_{12}$ é:

$\frac{\partial\left(\sigma_{c}^{2}\left(w^{*}\right) / \sigma_{2}^{2}\right)}{\partial \rho_{12}} \propto k \rho_{12}^{2}-\left(1+k^{2}\right) \rho_{12}+k$

A expressão (3.44) é um polinômio de sgunda ordem em $\rho_{12}$, com raízes:

$\frac{1+k^{2} \pm\left(1-k^{2}\right)}{2 k}=(k ; 1-k)$

Verifica-se que o ganho de diversificação será uma função crescente de $\rho_{12}$ e que gera a maior redução do erro de previsão para uma dada variação $\rho_{12}$ quando $\mathrm{k}=1$. 


\subsection{Métodos de Combinação de Previsões}

Os pesos das previsões dos modelos individuais podem ser determinados pelos seguintes métodos:

a) Combinações simples, em três categorias, conforme Clark e McCracken (2006):

- Média simples

- Mediana

- Média aparada

b) Pesos definidos pela raiz do erro quadrático médio

c) Pesos corrigidos pelo critério de informação de Akaike

d) Pesos determinados pelo método dos mínimos quadrados ordinários

e) Pesos determinados pelo critério mínimos quadrados sujeitos a restrições:

- Mínimos quadrados não negativos

- Mínimos quadrados igualmente restritos

- Mínimos quadrados igualmente restritos e não negativos

O conjunto de previsões individuais no instante t será denotado por $\mathrm{J}_{\mathrm{i}}()$ e o conjunto de modelos individuais é representado por $F_{i}()$, em que $\mathrm{i}=\{1, \ldots, \mathrm{M}\}$. Para cada modelo individual, pode haver h previsões à frente. De uma forma geral, para h passos à frente, cada modelo $F_{i}()$ gera $\mathrm{h}$ previsões $\left\{\hat{y}_{i, t-h+1}, \ldots, \hat{y}_{i, t-1}, \hat{y}_{i, t}\right\}$, denotadas por $\hat{y}_{i, t-j}$, onde $\mathrm{j}=\{\mathrm{h}-1, \ldots, 0\}$. Sem perda de generalidade, assume-se $\mathrm{h}=1$, pelo que cada modelo ajustado $\hat{F}_{i}()$ gera uma previsão em $\mathrm{t}$, denotada por $\hat{F}_{i}()=\hat{y}_{i, t}$. Os métodos são descritos no SAS Forecast Server Procedures 13.2.

\subsubsection{Combinações Simples}

\subsubsection{Média Simples}

Os pesos estimados para as previsões individuais $\mathrm{J}_{\mathrm{i}}()$ são dados por (3.43):

$$
\widehat{W}_{i}=\frac{1}{M}, \forall i \in \mathrm{J}_{\mathrm{i}}()
$$


A previsão combinada é dada por:

$\hat{y}_{c, t}=\frac{1}{M} \sum_{i=1}^{M} \hat{y}_{i, t}$

\subsubsection{Mediana}

A previsão combinada é estimada com base na mediana amostral das previsões individuais, ou seja:

$\hat{y}_{c, t}=\operatorname{mediana}\left\{\mathrm{J}_{\mathrm{i}}()\right\}, \forall i \in \mathrm{J}_{\mathrm{i}}()$

\subsubsection{Média Aparada}

Os pesos das previsões máxima e mínima são igualados a zero. Para os demais modelos, atribui-se o peso:

$$
\widehat{w}_{i}=\frac{1}{M-2}
$$

com a previsão combinada é dada por:

$\hat{y}_{c, t}=\frac{1}{M-2} \sum_{i=1}^{M-2} \hat{y}_{i, t}$

Entre os métodos de combinações simples, a média é a medida mais representativa de tendência central se as previsões pertencerem à mesma distribuição de probabilidade, caso contrário a mediana ou a média aparada podem fornecer a previsão combinada mais adequada. 


\subsubsection{Método da Raiz do Erro Quadrático Médio (REQM)}

Esse método usa o inverso do erro quadrático médio (REQM) da série de erros de previsão de cada modelo, com relação à soma do inverso do REQM de todos os modelos, como o peso de cada previsão individual. Portanto, quanto menor o RMSE, menor será o peso associado à previsão $\hat{y}_{i, t}$ do modelo $F_{i}()$, no tempo $\mathrm{t}$, onde $\mathrm{i}=\{1, \ldots, \mathrm{M}\}$.

$\mathrm{W}=\sum_{i=1}^{M} \frac{1}{R M S E_{i}}$

Portanto, o peso estimado para a previsão $\hat{y}_{i, t}$ do modelo $F_{i}()$ será:

$\widehat{W}_{i}=\frac{1 / \mathrm{RMSE}_{i}}{W}$

A previsão combinada em t será dada por:

$\hat{y}_{c, t}=\sum_{i=1}^{M} \widehat{w}_{i} \hat{y}_{i, t}$

\subsubsection{Método do Critério de Informação de Akaike (AIC)}

Esse método usa o critério de informação de Akaike (AIC) associado à série de erros de previsão de cada modelo como o peso de cada previsão individual.

Seja o AIC do modelo $F_{i}()$ dado por:

$\mathrm{AIC}_{\mathrm{i}}=\operatorname{AIC}\left(\hat{e}_{i, t-j} ; P_{i}\right), \hat{e}_{i, t-j}$ é a série do erro de previsão do modelo ajustado $\hat{F}_{i}() \mathrm{em} \mathrm{t}$, para $\mathrm{h}$ passos à frente, $\mathrm{j}=\{\mathrm{h}-1, \ldots, 0\}$ e $P_{i}$ é o número de parâmetros do modelo. Assumindo $\mathrm{h}=1, \mathrm{AIC}_{\mathrm{i}}$ $=\operatorname{AIC}\left(\hat{e}_{i, t} ; P_{i}\right)$.

Seja o menor $\mathrm{AIC}_{\mathrm{i}}$ denotado por $\mathrm{AIC}_{\min }$ e defina-se:

$\Delta_{i}=A I C_{i}-A I C_{\min }$ 
O peso estimado para a previsão $\hat{y}_{i, t}$ do modelo $F_{i}()$ será:

$\widehat{w}_{i}=\frac{\exp \left(-\frac{1}{2} \lambda \Delta_{i}\right)}{W}$,

$\mathrm{W}=M \sum_{i=1}^{M}\left[\exp \left(-\frac{1}{2} \lambda \Delta_{i}\right)\right]$ e $0 \leq \lambda$

Quando $0 \leq \lambda \leq 1$, o peso resultante reflete um valor entre o peso obtido pela média simples (quando $\lambda=0$ ) e o peso usual pelo AIC (quando $\lambda=1$ ). Quando $\lambda$ é elevado, por exemplo, 10 , o método tende a atribuir maior peso ao modelo com menor AIC. Frequentemente, a tendência de selecionar o melhor modelo é observada quando $\lambda=1$, a menos que outras previsões tenham $\mathrm{AIC}$ que são relativamente próximas a $\mathrm{AIC}_{\min }$.

A previsão combinada em t será dada por:

$\hat{y}_{c, t}=\sum_{i=1}^{M} \frac{\exp \left(-\frac{1}{2} \lambda \Delta_{i}\right)}{W} \hat{y}_{i, t}$

\subsubsection{Método de Mínimos Quadrados Ordinários (OLS)}

Os pesos são estimados por meio de regressão OLS de T valores observados da variável dependente $y_{t}$ sobre as $\mathrm{M}$ previsões individuais para cada instante $\mathrm{j}$ do período de estimação $\mathrm{T}$, onde $\mathrm{j}=\{1,2, \ldots \mathrm{T}\}$. Para o caso de previsão $\mathrm{h}$ passos à frente, tem-se que $\mathrm{t}=\mathrm{T}+\mathrm{h}$, onde $\mathrm{t}$ é o instante de tempo da última previsão.

A expressão geral de OLS é dada por:

$y_{t}=w_{0}+w_{1} \hat{y}_{1, t}+w_{2} \hat{y}_{2, t}+\cdots+w_{M} \hat{y}_{M, t}+\epsilon_{t}$, onde $\mathrm{t}=\{1,2, \ldots, \mathrm{T}\}$

A expressão (3.67) pode ser reescrita na notação matricial como:

$\mathbf{Y}=\widehat{\boldsymbol{Y}} \mathbf{W}+\boldsymbol{\epsilon}$ 
$\mathbf{Y}=\left[\begin{array}{c}y_{1} \\ y_{2} \\ \vdots \\ y_{t} \\ \vdots \\ y_{T}\end{array}\right]$, matriz T x 1 dos valores observados da variável dependente $y_{t}$

$\widehat{\boldsymbol{Y}}=\left[\begin{array}{ccccccc}1 & \hat{y}_{1,1} & \hat{y}_{2,1} & \cdots & \hat{y}_{i, 1} & \cdots & \hat{y}_{M, 1} \\ 1 & y_{1,2} & \hat{y}_{2,2} & \cdots & \hat{y}_{i, 2} & \cdots & \hat{y}_{M, 2} \\ \vdots & \vdots & \vdots & & \vdots & & \vdots \\ 1 & \hat{y}_{1, t} & \hat{y}_{2, t} & & \hat{y}_{i, t} & & \hat{y}_{M, t} \\ \vdots & \vdots & \vdots & & \vdots & & \vdots \\ 1 & \hat{y}_{1, T} & \hat{y}_{2, T} & \cdots & \hat{y}_{i, T} & \cdots & \hat{y}_{M, T}\end{array}\right]$, matriz T x $(M+1)$ das T previsões de cada

um dos M modelos individuais. A primeira coluna é referente aos valores das $\mathrm{M}$ constantes de cada modelo.

$\mathbf{W}=\left[\begin{array}{c}w_{0} \\ w_{1} \\ \vdots \\ w_{t} \\ \vdots \\ w_{M}\end{array}\right]$ é a matriz $(\mathrm{M}+1) \times 1$ composto por $w_{0}$ e pelos pesos de cada um dos M modelos individuais.

Os erros do modelo $\epsilon$ são denotados por:

$\boldsymbol{\epsilon}=\left[\begin{array}{c}\epsilon_{1} \\ \epsilon_{2} \\ \vdots \\ \epsilon_{t} \\ \vdots \\ \epsilon_{T}\end{array}\right]$ é a matriz $\mathrm{T}$ x 1 de erros normais e independentes com esperança $\mathrm{E}[\epsilon]=0$ e com

matriz de variância-covariância dada por

$\sigma^{2}\{\epsilon\}=\left[\begin{array}{cccc}\sigma^{2} & 0 & \cdots & 0 \\ 0 & \sigma^{2} & \cdots & 0 \\ \vdots & \vdots & & \vdots \\ 0 & 0 & \cdots & \sigma^{2}\end{array}\right]=\sigma^{2} \boldsymbol{I}$ 
A esperança do vetor $\mathbf{Y}$ é dada por:

$\mathrm{E}[\mathbf{Y}]=\widehat{\boldsymbol{Y}} \mathbf{W}$

e a matriz de variância-covariância de Y é a mesma de $\epsilon$ :

$\sigma^{2}\{\boldsymbol{Y}\}=\sigma^{2} \boldsymbol{I}$

O objetivo é determinar $\widehat{w}_{i}$ tal que minimize a soma dos erros quadrados:

$\min (\mathbf{Y}-\widehat{\boldsymbol{Y}} \mathbf{W})^{2}$

$\widehat{W}$ é o vetor com os parâmetros estimados de w:

$\widehat{\boldsymbol{W}}=\left[\begin{array}{c}\widehat{w}_{0} \\ \widehat{w}_{1} \\ \vdots \\ \widehat{w}_{t} \\ \vdots \\ \widehat{w}_{M}\end{array}\right]$

Os pesos estimados $\widehat{\boldsymbol{W}}$ são dados por:

$\widehat{\boldsymbol{W}}=\left(\widehat{\boldsymbol{Y}}^{\prime} \widehat{\boldsymbol{Y}}\right)^{-\mathbf{1}}\left(\widehat{\boldsymbol{Y}}^{\prime} \boldsymbol{Y}\right)$

ou, alternativamente, por:

$\widehat{w}_{i}=\sum_{t=1}^{T}\left(\hat{\boldsymbol{y}}_{t}^{\prime} \hat{\boldsymbol{y}}_{t}\right)^{-1} \sum_{t=1}^{T-1}\left(\hat{\boldsymbol{y}}_{t}^{\prime} y_{t}\right)$, onde $\mathrm{i}=\{1, \ldots, \mathbf{M}\}$

A previsão combinada em t será dada por:

$\hat{y}_{c, t}=\sum_{i=1}^{M} \widehat{w}_{i} \hat{y}_{i, t}$ 
Conforme Granger e Ramanathan (1984), três versões do método OLS podem ser consideradas:

$y_{t}=\sum_{i=1}^{M} \widehat{w}_{i} \hat{y}_{i, t}+\epsilon_{i, t}$, t.q $\sum_{i=1}^{M} \widehat{w}_{i}=1$

$y_{t}=c+\sum_{i=1}^{M} \widehat{w}_{i} \hat{y}_{i, t}+\epsilon_{i, t}$, t.q. $\sum_{i=1}^{M} \widehat{w}_{i}=1$

$y_{t}=c+\sum_{i=1}^{M} \widehat{w}_{i} \hat{y}_{i, t}+\epsilon_{i, t}$

A primeira equação é uma regressão restrita, onde a soma dos pesos devem somar a unidade e as previsões individuais não têm viés para assegurar que a combinação de previsões também não seja enviesada. A segunda equação é uma variante da primeira, onde as previsões individuais podem ter viés, que é corrigido pela introdução da constante c. A terceira equação é uma generalização das duas anteriores, onde a soma dos pesos não necessariamente é a unidade. A terceira versão geral do método OLS foi um dos métodos utilizados para a previsão combinada de preços de alumínio.

\subsubsection{Intervalo de Confiança de Combinação pelo Método de Mínimos Quadrados Ordinários (OLS)}

Seja o período de estimação $t=\{1,2, \ldots, T\}$ e sejam as previsões individuais em $T+h$, ou seja $\mathrm{h}$ passos à frente, para cada modelo individual $\mathrm{i}=\{1, \ldots, \mathrm{M}\}$, dadas por:

$\boldsymbol{Y}_{T+h}=\left\{\hat{y}_{1, T+h}, \hat{y}_{2, T+h}, \ldots, \hat{y}_{M, T+h}\right\}$

Em notação matricial:

$\widehat{\boldsymbol{Y}}_{T+h}=\left[\begin{array}{c}\hat{y}_{1, T+h} \\ \hat{y}_{2, T+h} \\ \vdots \\ \hat{y}_{M, T+h}\end{array}\right]$

A esperança da variável dependente $y_{t}, \mathrm{em} \mathrm{T}+\mathrm{h}$, a ser estimada é: 
$\mathrm{E}\left[Y_{T+h}\right]=\widehat{\boldsymbol{Y}}_{T+h}^{\prime} \boldsymbol{W}$

Portanto, o valor médio da variável resposta $y_{t}$ em T+h será:

$\widehat{Y}_{T+h}=\widehat{\boldsymbol{Y}}_{T+h}^{\prime} \widehat{\boldsymbol{W}}$

O estimador não é viesado pois:

$E\left[\widehat{Y}_{T+h}\right]=\widehat{\boldsymbol{Y}}_{T+h}^{\prime} \boldsymbol{W}=\mathrm{E}\left[Y_{T+h}\right]$

A variância de $\widehat{Y}_{T+h}$ é:

$\sigma^{2}\left[\widehat{Y}_{T+h}\right]=\sigma^{2} \widehat{\boldsymbol{Y}}_{T+h}^{\prime}\left(\widehat{\boldsymbol{Y}}^{\prime} \widehat{\boldsymbol{Y}}\right)^{-\mathbf{1}} \widehat{\boldsymbol{Y}}_{T+h}$

A variância também pode ser expressa como uma função da matriz de variância-covariância dos pesos estimados:

$\sigma^{2}\left[\widehat{Y}_{T+h}\right]=\widehat{\boldsymbol{Y}}_{T+h}^{\prime} \boldsymbol{\sigma}^{2}(\widehat{\boldsymbol{W}}) \widehat{\boldsymbol{Y}}_{T+h}$

A variância $\sigma^{2}\left[\widehat{Y}_{T+h}\right]$ é uma função das variâncias $\sigma^{2}\left(\widehat{W}_{l}\right)$ dos pesos $\widehat{W}_{i}$ e das covariâncias $\sigma^{2}\left(\widehat{W}_{i} \widehat{W}_{j}\right)$ entrre pares de pesos estimados.

Portanto, a variância estimada $s^{2}\left(\widehat{Y}_{T+h}\right)$ da combinação $\widehat{Y}_{T+h}$ em $\mathrm{T}+\mathrm{h}$ é dada por:

$S^{2}\left(\widehat{Y}_{T+h}\right)=\operatorname{MSE}\left(\widehat{\boldsymbol{Y}}_{T+h}^{\prime}\left(\widehat{\boldsymbol{Y}}^{\prime} \widehat{\boldsymbol{Y}}\right)^{-1} \widehat{\boldsymbol{Y}}_{T+h}\right)=\boldsymbol{Y}_{T+h}^{\prime} s^{2}(\widehat{\boldsymbol{W}}) \widehat{\boldsymbol{Y}}_{T+h}$

Os limites 1- $\alpha$ do intervalo de confiança para $\mathrm{E}\left[\widehat{Y}_{T+h}\right]$ são dados por, com $\mathrm{T}-\mathrm{M}+1$ graus de liberdade:

$$
\hat{Y}_{T+h} \pm t_{(1-\alpha / 2 ; T, M+1)} s\left(\widehat{Y}_{T+h}\right)
$$




\subsubsection{Métodos de Mínimos Quadrados Restritos}

Os três métodos dessa categoria são formulados como problemas de mínimos quadrados sujeitos a restrições e resolvidos por meio processo de otimização. Os três métodos minimizam a função objetiva:

$\mathrm{z}=\sum_{t=1}^{T}\left(y_{t}-\sum_{i=1}^{M} \widehat{w}_{i} \hat{y}_{i, t}\right)^{2}$

em que:

$y_{t}$ é o valor observado

$\widehat{w}_{i}$ é o peso para o modelo i

$\hat{y}_{i, t}$ é o valor estimado do modelo, no instante $\mathrm{t}$

Dependendo das restrições, têm-se os três métodos:

- Mínimos quadrados não negativos, que impõe a restrição:

$\widehat{w}_{i} \geq 0, i=1, \ldots, M$

- Mínimos quadrados igualmente restritos, que impõe a restrição:

$\sum_{i=1}^{M} \widehat{w}_{i}=1, i=1, \ldots, M$

- Mínimos quadrados igualmente restritos e não negativos, que impõe a restrição:

$$
\begin{aligned}
& \sum_{i=1}^{M} \widehat{w}_{i}=1, \\
& \widehat{w}_{i} \geq 0, i=1, \ldots, M
\end{aligned}
$$




\section{METODOLOGIA}

Este capítulo apresenta aspectos metodológicos, descrição e tratamento das variáveis, bem como a abordagem do uso de janelas móveis para estimação dos modelos individuais e combinados.

\subsection{Variável Dependente}

A variável dependente é o preço à vista de alumínio primário, determinado pela London Metal Exchange (LME). A série original incluiu 189 dados mensais (T=189), de janeiro de 1999 a setembro de 2014. Os dados mensais foram construidos a partir das médias diárias dos preços de fechamento divulgados oficialmente pela LME.

\subsubsection{Série Original de Preço à Vista de Alumínio (Valores Nominais)}

A Figura 1 mostra a série do preço à vista de alumínio em valores nominais, com uma média de US\$ 1.905 /t no período total de janeiro de 1999 a setembro de 2014. Notam-se preços crescentes de 2004 até um máximo de US\$ 3.071/t em julho de 2008, impulsionados principalmente pela demanda da China. A queda do preço do alumínio de $57 \%$ de julho de 2008 para $\$ 1.330$ por tonelada em fevereiro de 2009 é explicada fundamentalmente pela crise financeira global de 2008/09. Observa-se uma recuperação do preço até o primeiro trimestre de 2012, a partir do qual os efeitos da crise da Europa, associados a aumento dos estoques e um crescimento menor da demanda China (em torno de $8 \%$ comparado a uma taxa acima de $10 \%$ em períodos anteriores) levaram a uma nova queda de preços para US\$2.019 por tonelada em 2012 e, em seguida, para US\$ 1.846 por tonelada em 2013. De janeiro a setembro de 2014, a média do preço ficou em US\$1.800/t, ou 2\% abaixo da média anual de 2013. 


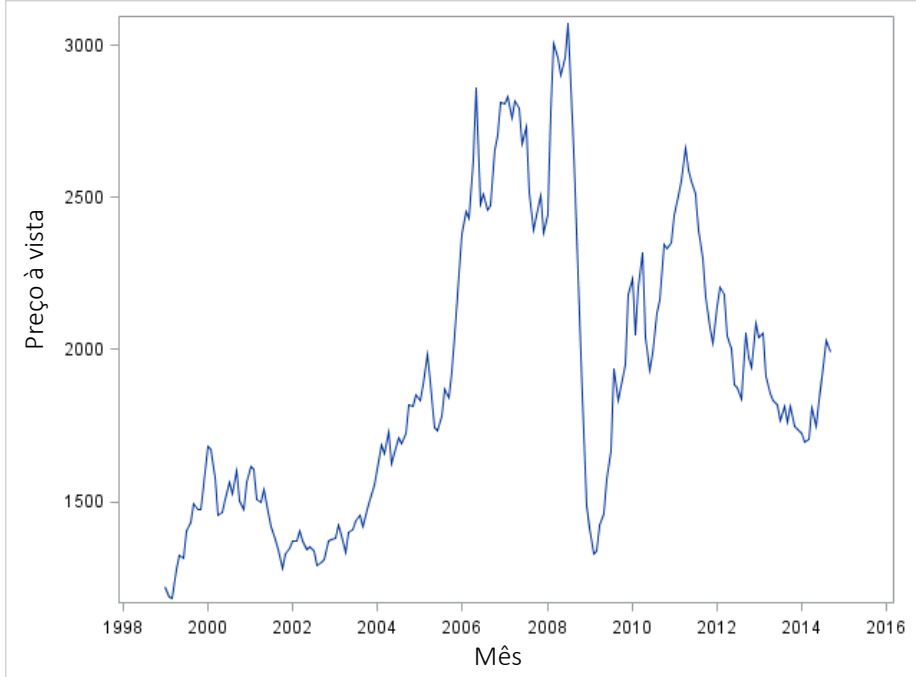

Figura 1 - Série Original do Preço à Vista de Alumínio (Valores Nominais, US\$/t) - Jan/1999 - Set/2014

Pela Figura 2, observa-se assimetria à esquerda. A análise dos dados indica que $63 \%$ das observações ficaram abaixo de US\$2.000/t, a uma média de US\$1.598/t. Apenas $15 \%$ dos dados ficaram acima de US\$2.500/t, a uma média de US\$2.485/t. Determinou-se também que $58 \%$ das observações ficaram abaixo da média global de US\$1.905/t na amostra completa. Pela Figura 3, vale ressaltar que os preços ficaram acima da média do período total (US\$ 1.905/t) apenas de cerca de 2006 ao inicío de 2008 e de 2010 ao início de 2012. As estatísticas descritivas (Tabela 1) indicam que o desvio-padrão foi de US\$ 475 por tonelada, resultando em um coeficiente de variação de $24,9 \%$. O teste de normalidade de Jarque-Bera indica que a série não segue uma distribuição normal, sendo assimétrica à esquerda e platicúrtica.

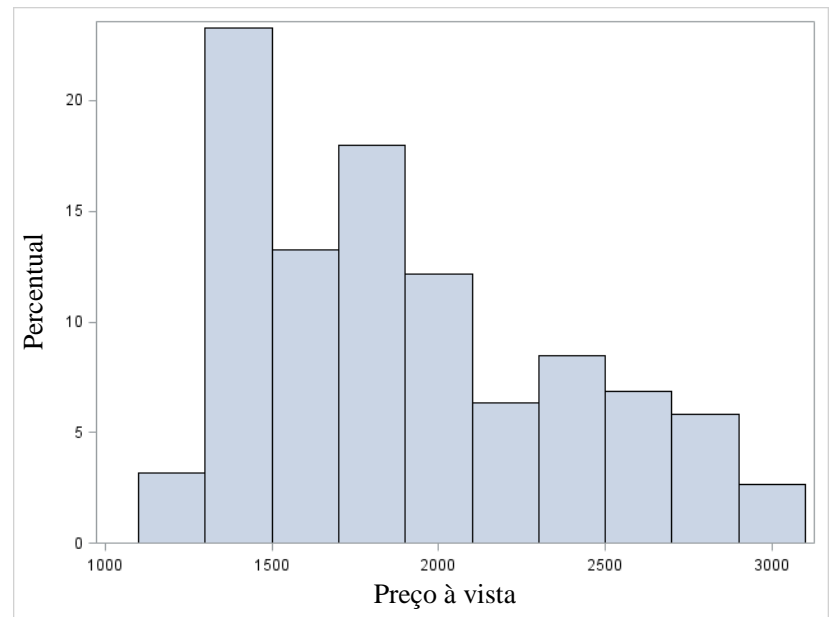

Figura 2 - Histograma do Preço à Vista de Alumínio (Valores Nominais, US\$/t)- Jan/1999 - Set/2014 


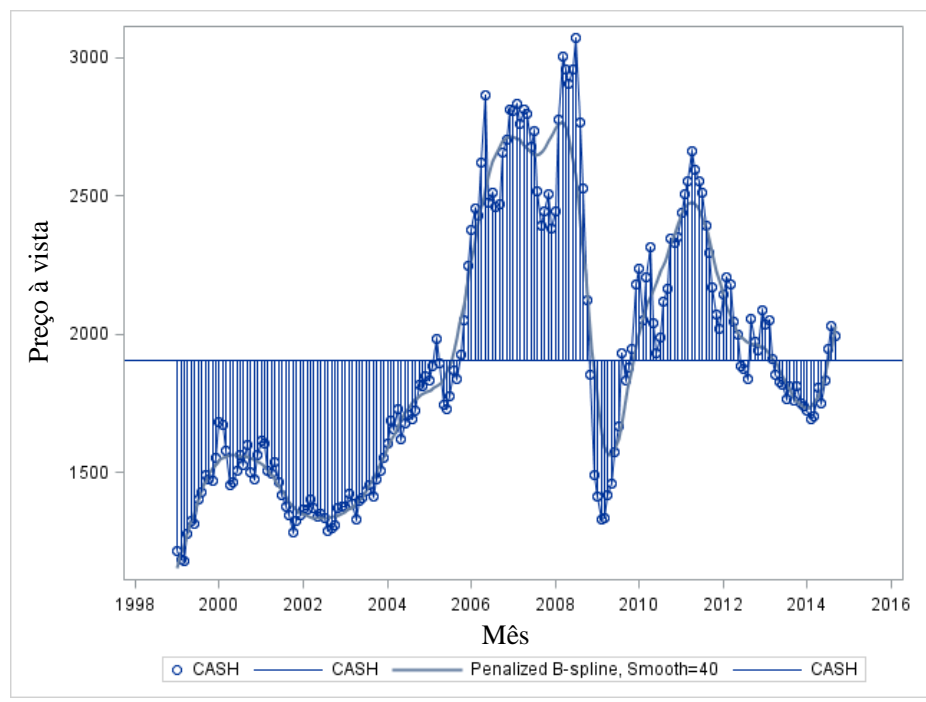

Figura 3 - Evolução do Preço à Vista de Alumínio (Valores Nominais, US\$/t), Jan/1999 - Set/2014

Tabela 1 - Estatísticas descritivas da série original de preço de alumínio

\begin{tabular}{lr}
\hline Descrição & \multicolumn{1}{c}{ Valor } \\
\hline Média & $1.905,26$ \\
Mediana & $1.830,57$ \\
Máximo & $3.071,24$ \\
Mínimo & $1.181,96$ \\
Desvio-padrão & 475,30 \\
Assimetria & 0,576 \\
Curtose & 2,301 \\
\hline Normalidade & \\
Estatística de Jarque-Bera & 14,293 \\
Probabilidade & 0,0007 \\
\hline \hline
\end{tabular}

\subsubsection{Série de Preço à Vista de Alumínio a Valores Constantes}

A fim de eliminar o efeito de inflação, a série original (CASH) foi deflacionada utilizando 3 índices, quais sejam: (i) o Consumer Price Index (CPI) dos Estados Unidos devido à relevância do mercado norte-americano no contexto global e no período analisado; (ii) o Producer Price Index (PPI) dos Estados Unidos, que reflete a produção industrial; (iii) o Non-Ferrous Metals Price Index $(N F)$ dos Estados Unidos, mas que inclui efeitos de outras commodities metálicas, como cobre e zinco. Os índices CPI e PPI foram obtidos do International Financial Statistics (IFS) do FMI. O NF foi obtido do United States Geological Survey (USGS). As séries deflacionadas estão a valores constantes com base na média anual de 2013. A Figura 4 mostra 
que as séries deflacionadas pelo CPI (CASH - CPI) e PPI (CASH - PPI) são semelhantes, enquanto a série deflacionada pelo NF (CASH - NF) ficou relativamente deslocada para baixo em função dos efeitos de preços de outros metais.

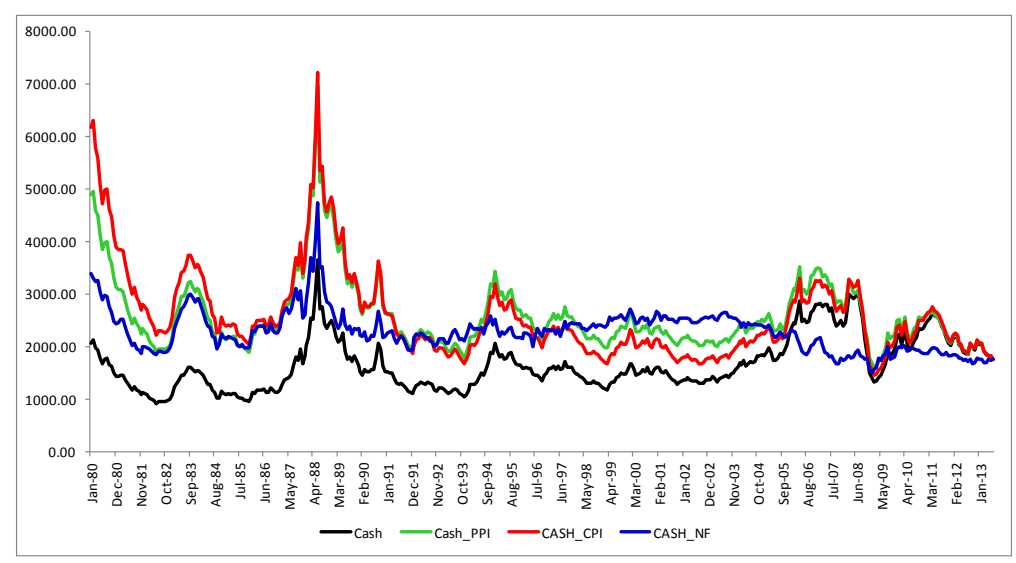

Figura 4 - Séries original e deflacionadas pelo CPI, PPI e NF de preço à vista de alumínio

Dada à abrangência do CPI e pelo fato do uso do NF refletir efeitos de preços de outros metais, utilizou-se a série deflacionada pelo CPI como base para as modelagens de preço. A Figura 5 mostra a série original (CASH) a valores nominais e a série a valores constantes (CASHR) de 2013 pelo CPI norte-americano.

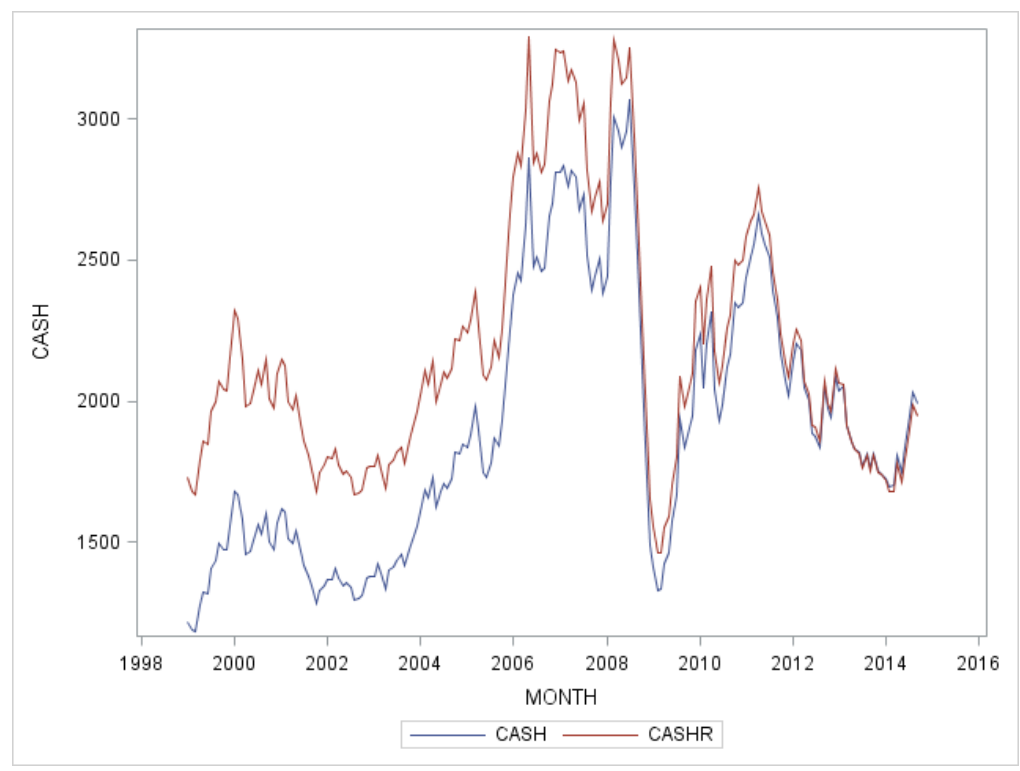

Figura 5 - Séries de preço à vista de alumínio em valores nominais e constantes deflacionado pelo CPI 
Na Figura 6, o correlograma da série de preço deflacionado de alumínio apresenta maiores autocorrelações para as defasagens iniciais, de 1 a 8 . A função de autocorrelação parcial apresenta autocorrelação significativa na primeira defasagem. A Tabela 2 confirma que a série de preço deflacionado de alumínio apresenta correlação serial. A análise do teste de raiz unitária ADF (Tabela 3) demonstra que a série não é estacionária em nenhum dos casos.
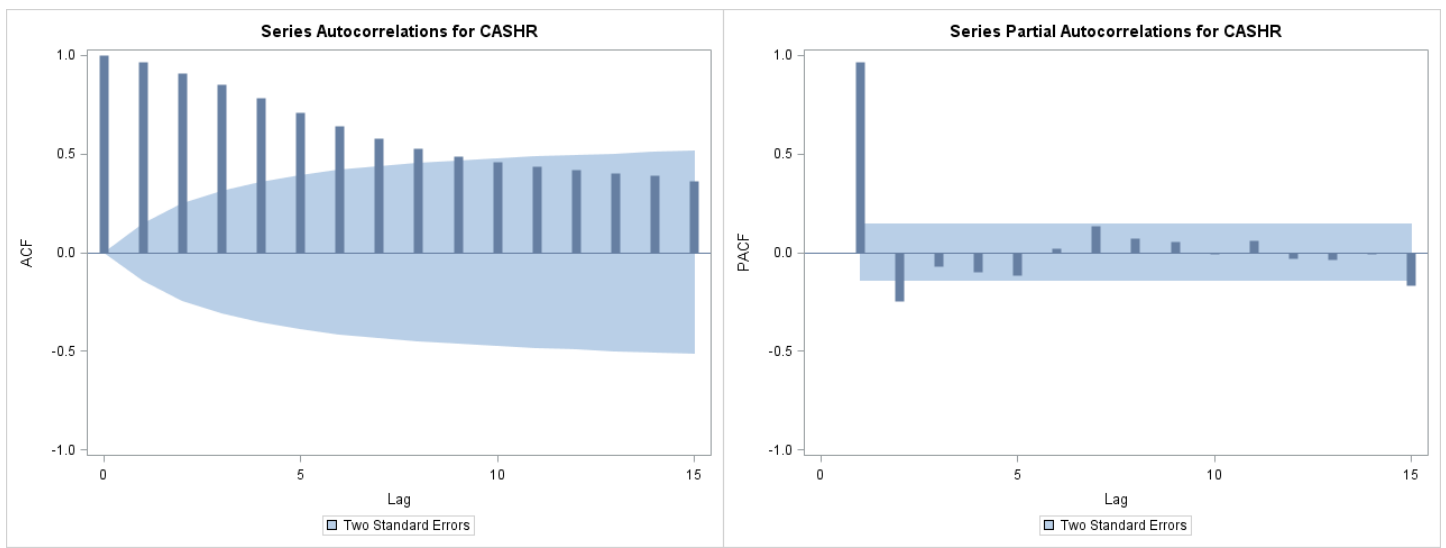

Figura 6 - Função de Autocorrelação e Autocorrelação Parcial da Série Deflacionada de Preço de Alumínio

Tabela 2 - Teste de Portmanteau para a Série de Preço Deflacionado de Alumínio

\begin{tabular}{crrrrrrrr}
\hline \multicolumn{7}{c}{ Teste de Autocorrelações Serial (Portmanteau) } \\
\hline Defasagem & Chi-Square & \multicolumn{1}{c}{ DF } & \multicolumn{7}{c}{ Pr $>$ ChiSq } \\
\hline $\mathbf{6}$ & 777.63 & $6<.0001$ & 0.963 & 0.91 & 0.85 & 0.784 & 0.711 & 0.638 \\
$\mathbf{1 2}$ & 1064.37 & $12<.0001$ & 0.578 & 0.529 & 0.49 & 0.457 & 0.436 & 0.42 \\
\hline
\end{tabular}

Tabela 3 - Teste de Raiz Unitária ADF para a Série de Preço Deflacionado de Alumínio

\begin{tabular}{|c|c|c|c|c|c|c|c|c|}
\hline Tipo & Lags & Rho & ProbRho & Tau & ProbTau & FValue & ProbF & Variável \\
\hline \multirow[t]{3}{*}{ Sem constante, sem tendência } & 0 & -0.175 & 0.642 & -0.244 & 0.598 & - & - & CASHR \\
\hline & 1 & -0.322 & 0.609 & -0.349 & 0.558 & - & - & CASHR \\
\hline & 2 & -0.343 & 0.604 & -0.361 & 0.553 & - & - & CASHR \\
\hline \multirow[t]{3}{*}{ Com constante, sem tendência } & 0 & -6.679 & 0.292 & -1.890 & 0.336 & 1.795 & 0.613 & CASHR \\
\hline & 1 & -11.251 & 0.095 & -2.416 & 0.139 & 2.927 & 0.325 & CASHR \\
\hline & 2 & -12.640 & 0.067 & -2.512 & 0.114 & 3.163 & 0.265 & CASHR \\
\hline \multirow[t]{3}{*}{ Com constante, com tendência } & 0 & -6.437 & 0.704 & -1.801 & 0.701 & 1.904 & 0.797 & CASHR \\
\hline & 1 & -11.012 & 0.360 & -2.343 & 0.408 & 2.961 & 0.586 & CASHR \\
\hline & 2 & -12.375 & 0.284 & -2.440 & 0.357 & 3.187 & 0.541 & CASHR \\
\hline
\end{tabular}




\subsubsection{Série Transformada de Preço de Alumínio}

A fim de tornar a série estacionária para o ajuste dos modelos ARIMA e ARMAX, utilizou-se a série transformada do retorno $\mathrm{Y}_{\mathrm{t}}$, dada pela primeira diferença dos logaritmos naturais do preço a valor constante de alumínio, dada pela expressão (4.1).

$\mathrm{Y}_{\mathrm{t}}=\ln \left(\mathrm{Pt} / \mathrm{P}_{\mathrm{t}-1}\right)=\ln \left(\mathrm{P}_{\mathrm{t}}\right)-\ln \left(\mathrm{P}_{\mathrm{t}-1}\right)$

A série transformada $Y_{t}$ está apresentada na Figura 7. Os testes de raiz unitária ADF confirmam a estacionariedade da série, conforme mostrado na Tabelas 4.

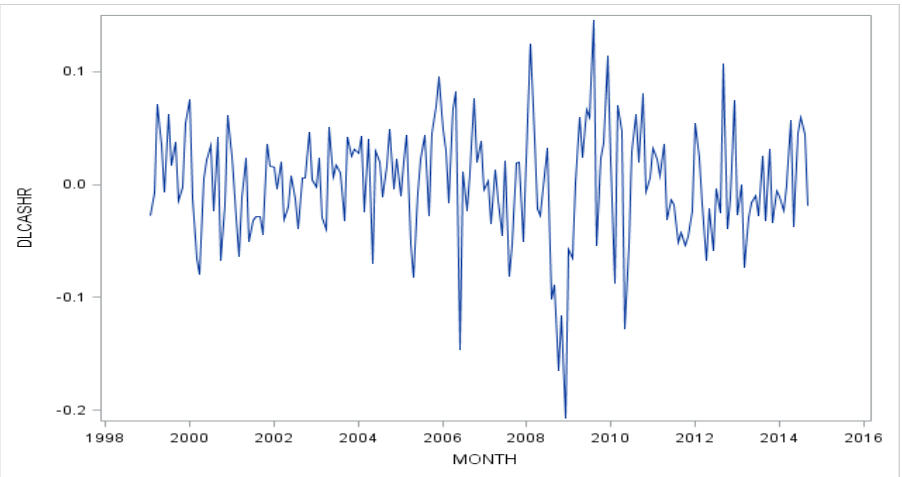

Figura 7 - Série Transformada do Retorno do Preço de Alumínio

Tabela 4 - Teste de Raiz Unitária ADF para a Série Transformada do Retorno do Preço de Alumínio

\begin{tabular}{lrrrrrrrr}
\hline Tipo & Lags & Rho & ProbRho & Tau & ProbTau & FValue & ProbF & Variável \\
\hline Sem constante, sem tendência & 0 & -138.431 & 0.000 & -10.459 & 0.000 & - & - & DLCASHR \\
& 1 & -122.614 & 0.000 & -7.789 & 0.000 & - & - & DLCASHR \\
& 2 & -101.623 & 0.000 & -6.276 & 0.000 & - & - & DLCASHR \\
\hline \multirow{3}{*}{ Com constante, sem tendência } & 0 & -138.464 & 0.000 & -10.433 & 0.000 & 54.428 & 0.001 DLCASHR \\
& 1 & -122.667 & 0.000 & -7.770 & 0.000 & 30.183 & 0.001 DLCASHR \\
& 2 & -101.640 & 0.000 & -6.259 & 0.000 & 19.588 & 0.001 DLCASHR \\
\hline \multirow{3}{*}{ Com constante, com tendência } & 0 & -138.946 & 0.000 & -10.440 & 0.000 & 54.500 & 0.001 DLCASHR \\
& 1 & -123.720 & 0.000 & -7.784 & 0.000 & 30.297 & 0.001 DLCASHR \\
& 2 & -102.696 & 0.000 & -6.254 & 0.000 & 19.580 & 0.001 DLCASHR \\
\hline
\end{tabular}




\subsection{Variáveis Independentes}

O preço de alumínio está associado a fundamentos de mercado, ou seja, oferta, demanda e estoques; a preços futuros; a condições macroeconômicas, como produção industrial, uma vez que se trata de um setor como ampla gama de aplicações industriais; à taxa de câmbio, pois os preços são denominados em dólares parte dos custos em moeda local; e a variáveis financeiras, como taxa de juros e mercado de ações, uma vez que agentes de mercado tomam posições vendidas ou compradas para fins de investimento financeiro.

A Tabela 5 mostra 30 variáveis independentes, inicialmente analisadas para o ajuste do modelo ARMAX. As 30 variáveis foram agrupadas em 8 categorias, quais sejam, (1) preços; (2) produção industrial; (3) produção; (4) consumo; (5) estoque; (6) câmbio; (7) taxa de juros; (8) mercado de ações. Realizaram-se 1.110 transformações a partir dessas 30 variáveis, sendo 390 transformações em nível (13 por variável) e 720 em diferenças (24 por variável). As Tabelas 6 e 7 mostram, respectivamente, os totais de variáveis analisadas e os tipos de transformações.

Tabela 5 - Variáveis Independentes Analisadas

\begin{tabular}{|c|c|c|c|c|c|}
\hline Item & Categoria & Descrição & Fonte & Unidade & Variável \\
\hline 1 & Preços & Preço futuro de 3 meses de alumínio, deflacionado pelo CPI (EUA) & LME, IMF & US\$/t & M3R \\
\hline 2 & & Preço do barril de petóleo cru, tipo Brent & IMF & US\$/t & BRENT \\
\hline 3 & & Consumer Price Index dos EUA (Base 100 = média de 2013) & Harver Analytics & Índice & CPI2013 \\
\hline 4 & & Índide de preços de metais não ferrosos nos EUA & Harver Analytics & Índice & NFINDEX \\
\hline 5 & & Índice de preço para a indústria de produtos não ferrosos nos EUA & Harver Analytics & Índice & USPPINFM \\
\hline 6 & Produção industrial & Índice de produção industrial dos Estados Unidos & Harver Analytics & Índice & USIP \\
\hline 7 & & Índice de produção de metais primários dos Estados Unidos & Harver Analytics & Índice & USPMIP \\
\hline 8 & & Índie de produção industrial da China & Harver Analytics & Índice & CHIP \\
\hline 9 & & Índide de produção industrial da Europa & Harver Analytics & Índice & EURIP \\
\hline 10 & & Índice de produção industrial do Japão & IMF & Índice & JAPIP \\
\hline 11 & Produção de alumínio primário & Capacidade global de produção de alumínio primário & CRU & $\mathrm{milt}$ & CAP \\
\hline 12 & & Produção global de alumínio primário & CRU & milt & GLPROD \\
\hline 13 & & Capacidade de utilização de aluminio primário & CRU & milt & GLCAPUT \\
\hline 14 & Consumo de alumínio primário & Consumo global de alumínio primário & CRU & milt & GLCONS \\
\hline 15 & & Consumo global de alumínio primário, excluindo a China & CRU & milt & EXCONS \\
\hline 16 & Estoques de alumínio primário & Dias de estoques globais de alumínio primário & CRU & dias & GLTOTDOC \\
\hline 17 & & Dias de estoques globais reportados de alumínio primário & CRU & dias & GLREPDOC \\
\hline 18 & & Dias de estoques globais reportados, excluindo a China & CRU & dias & EXREPDO \\
\hline 19 & & Dias de estoque na London Metal Exchange & CRU & dias & EXLMEDOC \\
\hline 20 & & Estoques globais de alumínio primário & CRU & mil t & GLSTOCK \\
\hline 21 & & Estoques globais reportados de alumínio primário & CRU & milt & GLREPSTO \\
\hline 22 & & Estoques globais de alumínio primário, excluindo a China & CRU & milt & ЕХTOTSTO \\
\hline 23 & & Estoques reportados de alumínio primário, excluindo a China & CRU & milt & EXREPSTO \\
\hline 24 & & Estoques de alumínio primário mantidos pela London Metal Exchange & CRU & milt & LMESTOCK \\
\hline 25 & Câmbio & Taxaefetiva de câmbio do dólar americano vs cesta de moedas (Base 1973=100) & Harver Analytics & Índice & FXUSD \\
\hline 26 & & Taxa efetiva de câmbio do Euro (Base 2005=100) & Harver Analytics & Índice & FXEUREFF \\
\hline 27 & & Taxa de câmbio do yen japonês & Harver Analytics & Yen/USS & FXJAP \\
\hline 28 & Taxa de juros & Taxa de juros da Libor de 3 meses (\% ao ano) & Harver Analytics & \% a.a. & LIBOR \\
\hline 29 & Mercado de ações & Indice Dow-Jones nos Estados Unidos & Harver Analytics & Indice & DJ \\
\hline 30 & & Índice Standard-Poors das 500 maiores empresas nos EUA & Harver Analytics & Índice & SP500 \\
\hline
\end{tabular}


Tabela 6 - Total de transformações das variáveis independentes

\begin{tabular}{lccc}
\hline Qtde. Variáveis & Nível & Diferença & Total \\
\hline Transformação por variável & 13 & 24 & 37 \\
Total de transformações & 390 & 720 & 1.110 \\
Total de variáveis base & & & 30 \\
& & & 1.140 \\
\hline
\end{tabular}

Tabela 7 - Tipo de transformação por variável

\begin{tabular}{lclc}
\hline \multicolumn{2}{c}{ Transformações em Nível por Variável } & \multicolumn{2}{c}{ Transformações em Diferenças por Variável } \\
\hline Descrição & Qtde. & Descrição & Qtde. \\
\hline Lag variável (1 a 12 meses) & 12 & Diferença da base (1 a 12 lags) & 12 \\
& & Variação percentual da bse (1 a 6 lags) & 6 \\
Logaritmo natural & 1 & Diferença de logaritmo (1 a 6 lags) & 6 \\
\hline Total & $\mathbf{1 3}$ & & $\mathbf{2 4}$ \\
\hline
\end{tabular}

\subsection{Amostras Utilizadas}

\subsubsection{Amostra Utilizada para Seleção dos Modelos Individuais}

Utilizou-se a amostra inteira, de janeiro de 1999 a setembro de 2014 para a seleção dos modelos individuais por meio do critério de informação de Schwartz, conforme descrito na seção 5.1. Cada modelo individual foi subsequentemente ajustado tendo como período de estimação uma janela móvel de 36 meses, conforme descrito na seção 4.3.2.

\subsubsection{Amostra Utilizada para Ajuste dos Modelos Individuais e Combinados e para Projeções Fora da Amostra}

A amostra utilizada para ajuste dos parâmetros de cada modelo individual foi uma janela móvel de 36 meses, considerando que os últimos três anos constituem um período relevante para a perspectiva da indústria alumínio no ano subsequente.

A Figura 8 ilustra a seleção das amostras para ajustes dos modelos individuais. A primeira amostra foi de janeiro de 1999 a dezembro de 2001. A segunda amostra foi de feveiro de 1999 a janeiro de 2002. A última amostra (a mais recente, que foi a de número 142) foi de outubro 
de 2010 a setembro de 2013. Dessa forma, 142 amostras foram utilizadas para ajustes de cada modelo individual.

Para cada uma das 142 amostras, foram realizadas 9 combinações de modelos. Assim, foram ajustados 9 modelos combinados para a primeira amostra, de janeiro de 1999 a dezembro de 2001. Em seguida, 9 modelos combinados para a segunda amostra, de fevereiro de 1999 a janeiro de 2002. Por fim, 9 modelos combinados para a última amostra $\left(142^{\mathrm{a}}\right)$, de outubro de 2010 a setembro de 2013.

Para cada um dos ajustes dos modelos individuais e combinados, tomou-se o $12^{\circ}$ preço de alumínio projetado, 12 meses à frente. Dessa forma, foram obtidos 142 previsões de preço de alumínio fora da amostra. O primeiro preço projetado correspondeu a dezembro de 2002 . O segundo preço projetado, a janeiro de 2013. O último preço projetado correspondeu a setembro de 2014.

Os 142 pontos projetados 12 meses à frente, fora da amostra, foram utilizados para a comparação do poder preditivo dos modelos indiviuais e combinados.

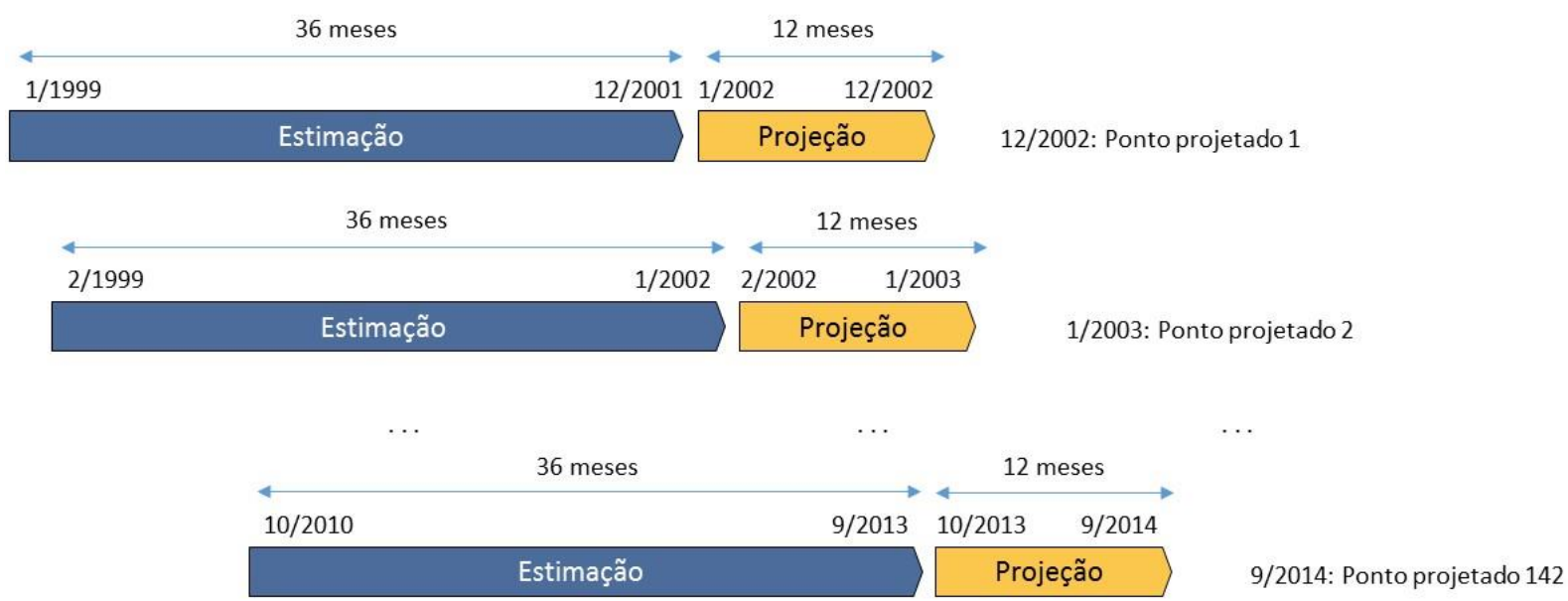

Figura 8 - Amostra Utilizada para Ajuste dos Parâmetros dos Modelos Individuais 


\section{$5 \quad$ RESULTADOS}

Este capítulo apresenta os resultados dos modelos individuais ajustados, combinações das previsões, e a seleção do melhor conjunto de modelos, dado um nível de confiança de 0.1, por meio do Model Confidence Set. A programação para ajustes e projeções dos modelos individuais e combinados foi realizada no ambiente do software SAS 9.4, incluindo os módulos ETS e Forecast Server. A comparação do poder preditivo dos modelos pelo Model Confidence Set foi realizada por meio do módulo MULCOM, do software OxMetrics 6.

\subsection{Modelos Individuais}

Para a projeção da série CASHR, foram ajustados cinco modelos individuais, a serem analisados nas seções seguintes.

1. $\mathrm{AR}(1)$

2. ARMA $(1,10)$

3. ARMAX 1

4. ARMAX 2

5. Modelo Estrutural

\subsubsection{Modelo AR(1)}

O modelo AR(1) foi ajustado para fins de benchmarking. A Tabela 8 mostra os resultados na estimação da amostra completa (janeiro/1999 a setembro/2014, conforme explicado na seção 4.3.2) e a Tabela 9 apresenta estatísticas de ajuste do modelo.

Tabela 8 - Estimação do Modelo AR(1)

\begin{tabular}{cccccc}
\hline Variável & Parâmetro & Estimativa & Erro-Padrão & $\mathrm{t}$ & Nível Descritivo \\
\hline CASHR & AR(1) & 0,25973 & 0,07065 & 3,68 & 0,0003 \\
\hline
\end{tabular}


Tabela 9 - Estatísticas de Ajuste do Modelo AR(1)

\begin{tabular}{cc}
\hline Estatística & Valor \\
\hline AIC & $1.782,6157$ \\
SBC & $1.785,6157$ \\
RMSE & 113,9409 \\
MAPE & 3,7819 \\
MAE & 84,2182 \\
R-Quadrado & 0,9361 \\
R-Quadrado Ajustado & 0,9361 \\
\hline
\end{tabular}

A análise de correlação dos resíduos (Figura 9) mostra que não há autocorrelação diferente de zero, indicando adequação do modelo. A análise da normalidade (Figura 10) indica que a distribuição dos resíduos aproxima-se de uma distribuição normal. A Figura 11 aponta que os resultados estimados dentro da amostra estão dentro do intervalo de confiança de $95 \%$. Os preços projetados, 12 meses à frente por meio da janela móvel de estimação de 36 meses, estão apresentados na Figura 12, juntamente com os valores observados. A Tabela 10 apresenta o resultado do teste de normalidade de Jarque-Bera. Como o nível descritivo é menor que 0,05, conclui-se que os resíduos seguem uma distribuição normal.

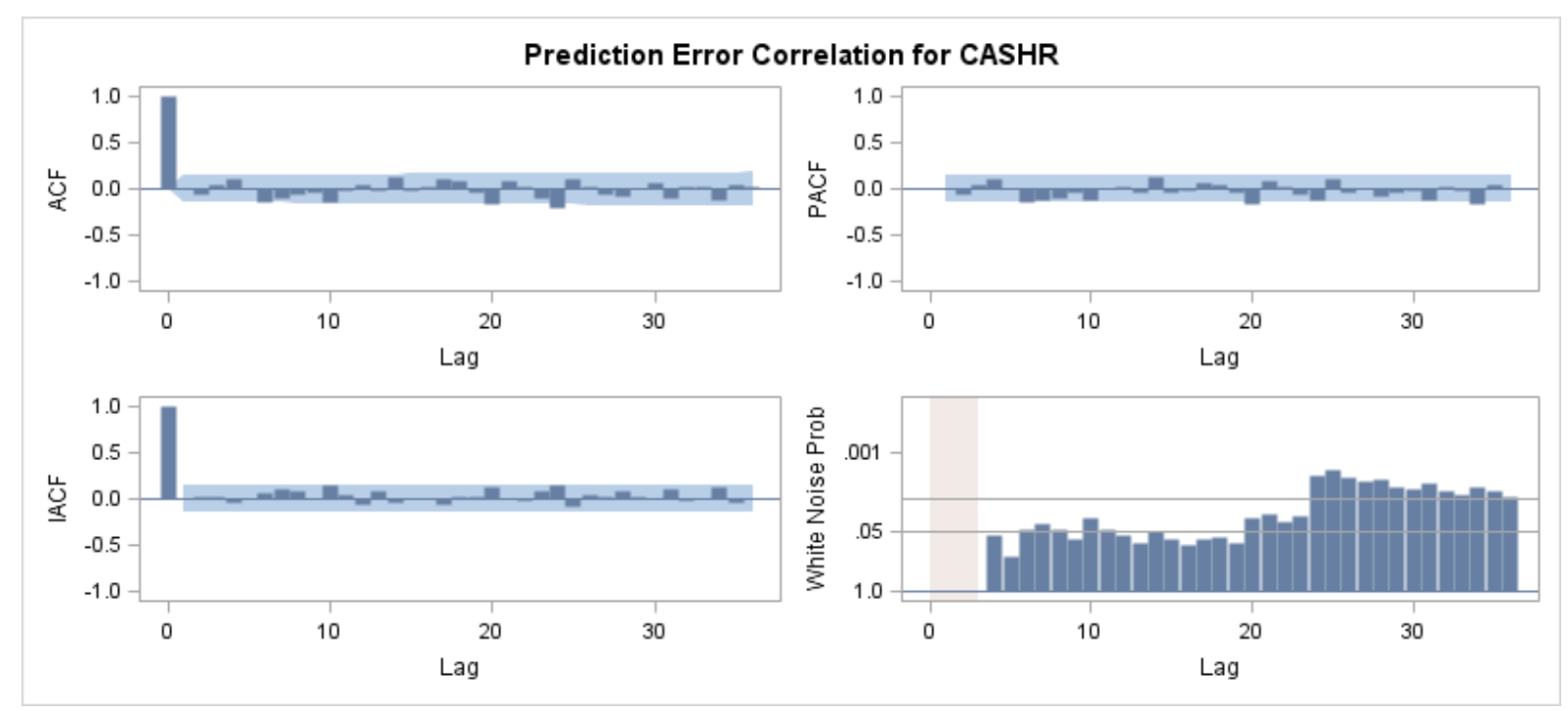

Figura 9 - Análise de Correlação dos Resíduos do Modelo AR(1) 


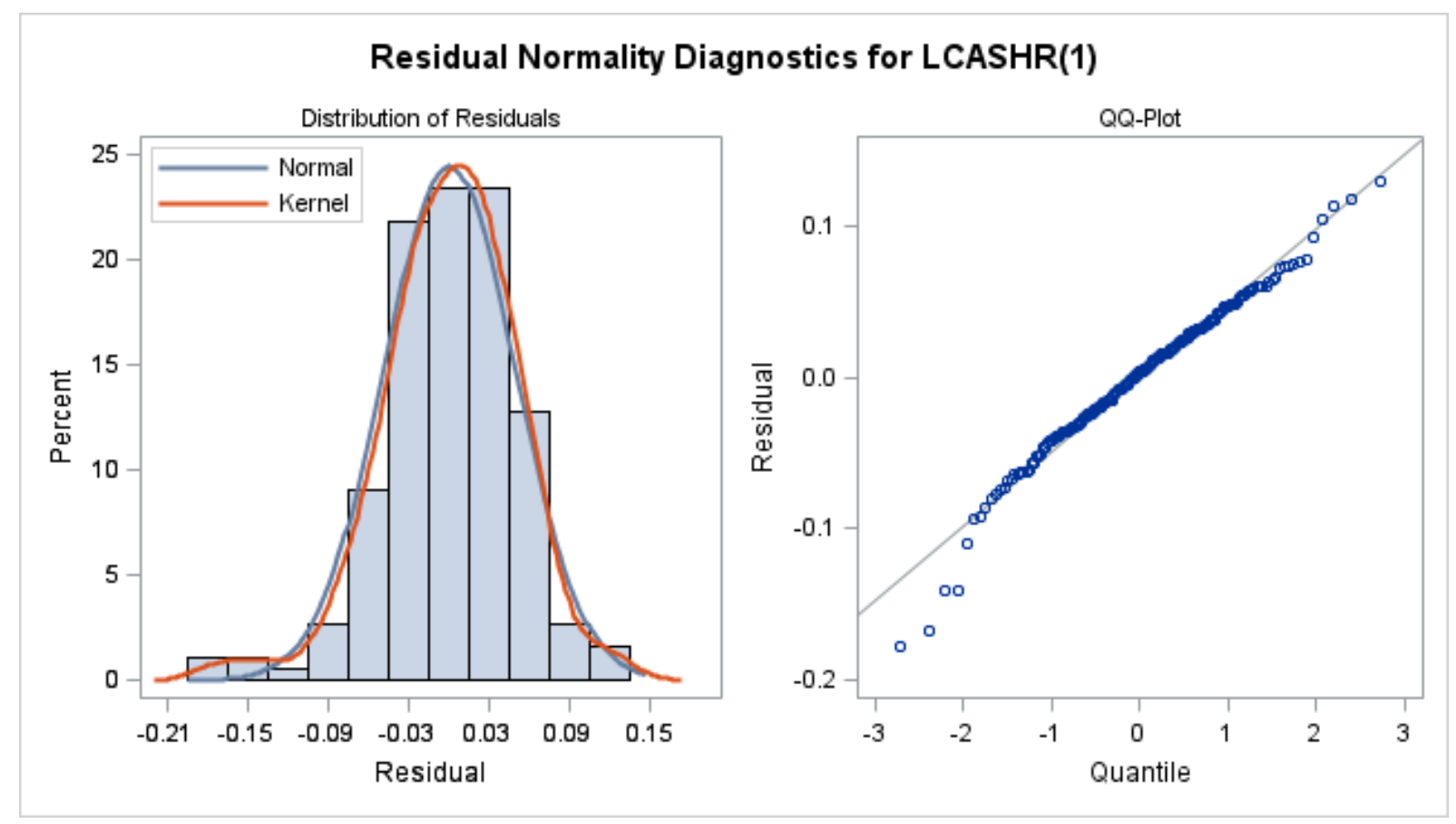

Figura 10 - Análise de Normalidade dos Resíduos do Modelo AR(1)

Tabela 10 - Teste de Normalidade de Jarque-Bera - Modelo AR(1)

\begin{tabular}{cc}
\hline Estatística Jarque-Bera & Nível Descritivo \\
\hline 20,3128 & 0,000038 \\
\hline
\end{tabular}

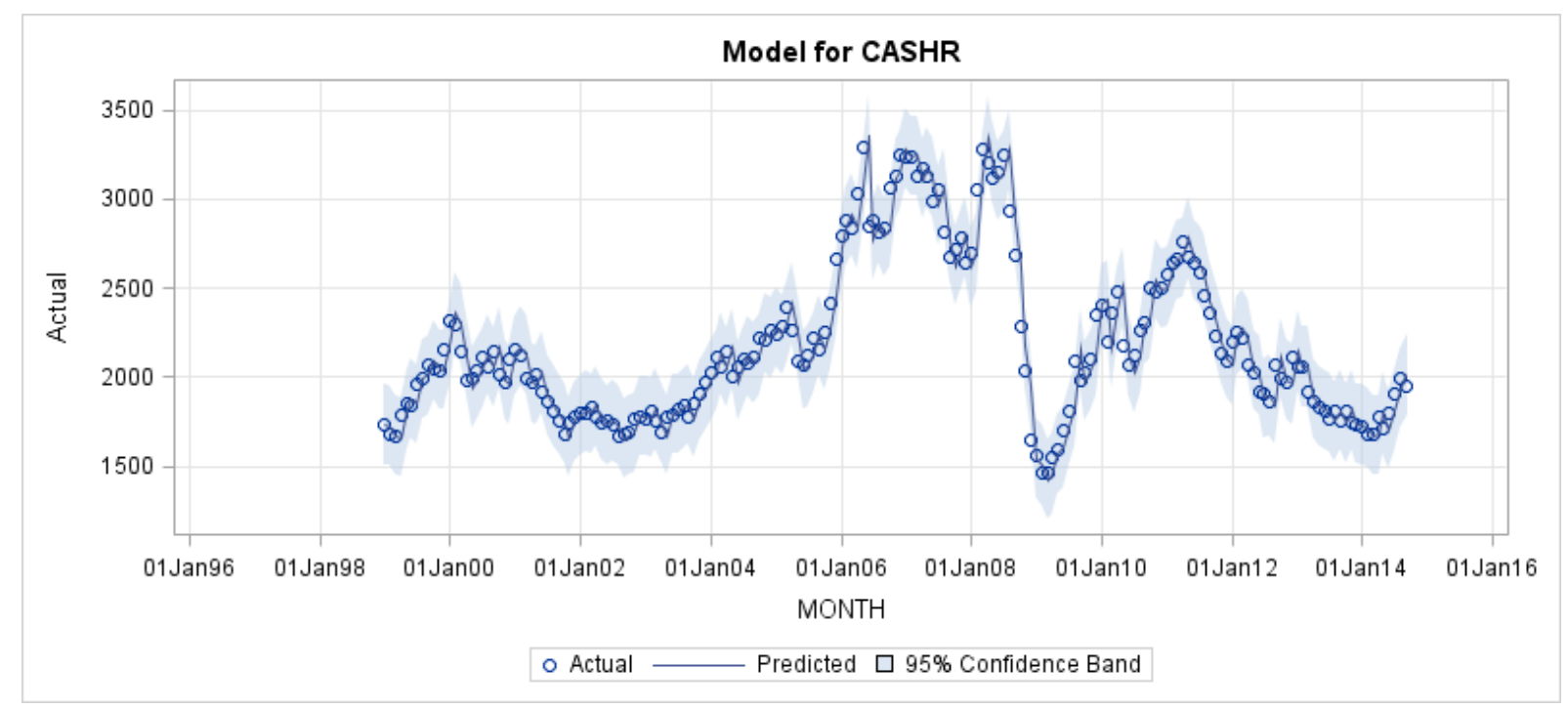

Figura 11 - Modelo AR(1) Ajustado dentro da Amostra Completa 


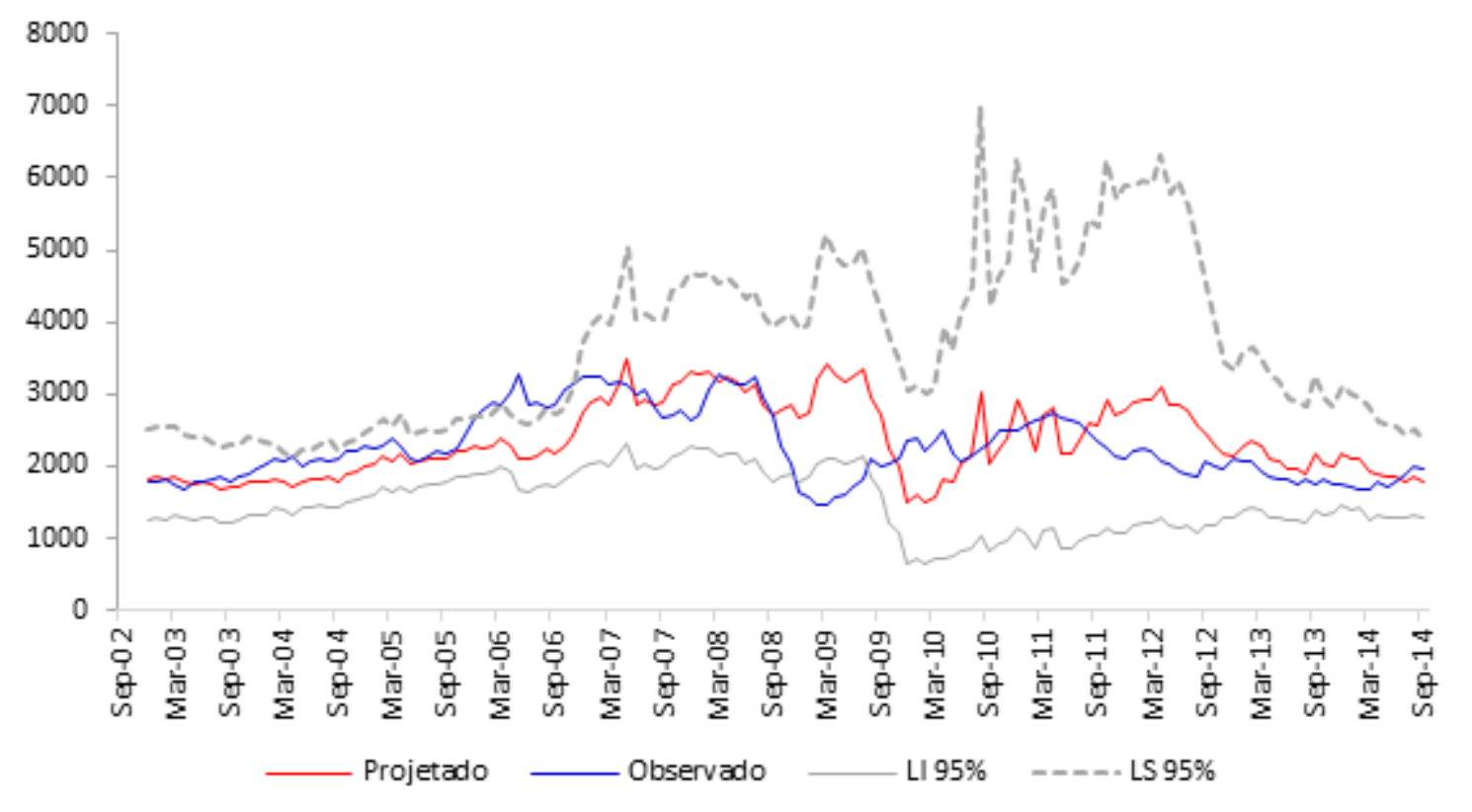

Figura 12 - Modelo AR(1): Preços de Alumínio Observados e Projetados, 12 Meses à Frente Ponto a Ponto, Fora da Amostra (Dez/2002 - Set/2014, US\$/t)

\subsubsection{Modelo ARMA(1,10)}

O modelo $\operatorname{ARMA}(1,10)$ foi o que apresentou os menores critério de informação de Akaike e Schwartz, após o ajuste de todas as combinações ARMA possíveis com p e q variando de 0 a 12. A Tabela 10 apresenta os 6 melhores modelos, ordenados pelos critérios de Akaike (AIC) e Schwartz (SBC).

Tabela 11 - Modelos ARMA com Menores Critérios de Informação (Akaike, SBC)

\begin{tabular}{cccccc}
\hline AIC & $\mathrm{p}(\mathrm{AIC})$ & $\mathrm{q}(\mathrm{AIC})$ & $\mathrm{SBC}$ & $\mathrm{p}(\mathrm{SBC})$ & $\mathrm{q}(\mathrm{SBC})$ \\
\hline $1.777,6006$ & 1 & 10 & $1.784,1135$ & 1 & 10 \\
$1.779,7783$ & 1 & 6 & $1.786,2512$ & 0 & 1 \\
$1.780,2251$ & 10 & 1 & $1.786,6980$ & 10 & 1 \\
$1,781,9665$ & 1 & 4 & $1.788,4394$ & 1 & 0 \\
$1.782,3318$ & 1 & 7 & $1.788,8047$ & 1 & 4 \\
\hline
\end{tabular}

A Tabela 12 mostra os resultados na estimação da amostra completa (janeiro/1999 a setembro/2014) e a Tabela 13 apresenta estatísticas de ajuste do modelo. 
Tabela 12 - Estimação do Modelo ARMA $(1,10)$

\begin{tabular}{cccccc}
\hline Variável & Parâmetro & Estimativa & Erro-Padrão & $\mathrm{t}$ & Nível Descritivo \\
\hline CASHR & AR(1) & 0,24202 & 0,07147 & 3,39 & 0,0009 \\
\hline CASHR & MA(10) & 0,23123 & 0,07323 & 3,16 & 0,0019 \\
\hline
\end{tabular}

Tabela 13 - Estatísticas de Ajuste do Modelo ARMA(1, 10)

\begin{tabular}{cc}
\hline Estatística & Valor \\
\hline AIC & $1.777,6006$ \\
SBC & $1.784,1135$ \\
MAPE & 111,8467 \\
MAE & 3,7391 \\
R-Quadrado & 83.3744 \\
R-Quadrado Ajustado & 0,9384 \\
\hline
\end{tabular}

A análise de correlação dos resíduos (Figura 13) mostra que não há autocorrelação diferente de zero, indicando adequação do modelo. A análise de normalidade (Figura 14) indica que a distribuição dos resíduos aproxima-se de uma distribuição normal. A Figura 15 aponta que os resultados estimados dentro da amostra estão dentro do intervalo de confiança de $95 \%$. Os preços projetados, 12 meses à frente por meio da janela móvel de estimação de 36 meses, estão apresentados na Figura 16, juntamente com o valores observados. A Tabela 14 apresenta o resultado do teste de normalidade de Jarque-Bera. Como o nível descritivo é menor que 0,05, conclui-se que os resíduos seguem uma distribuição normal.

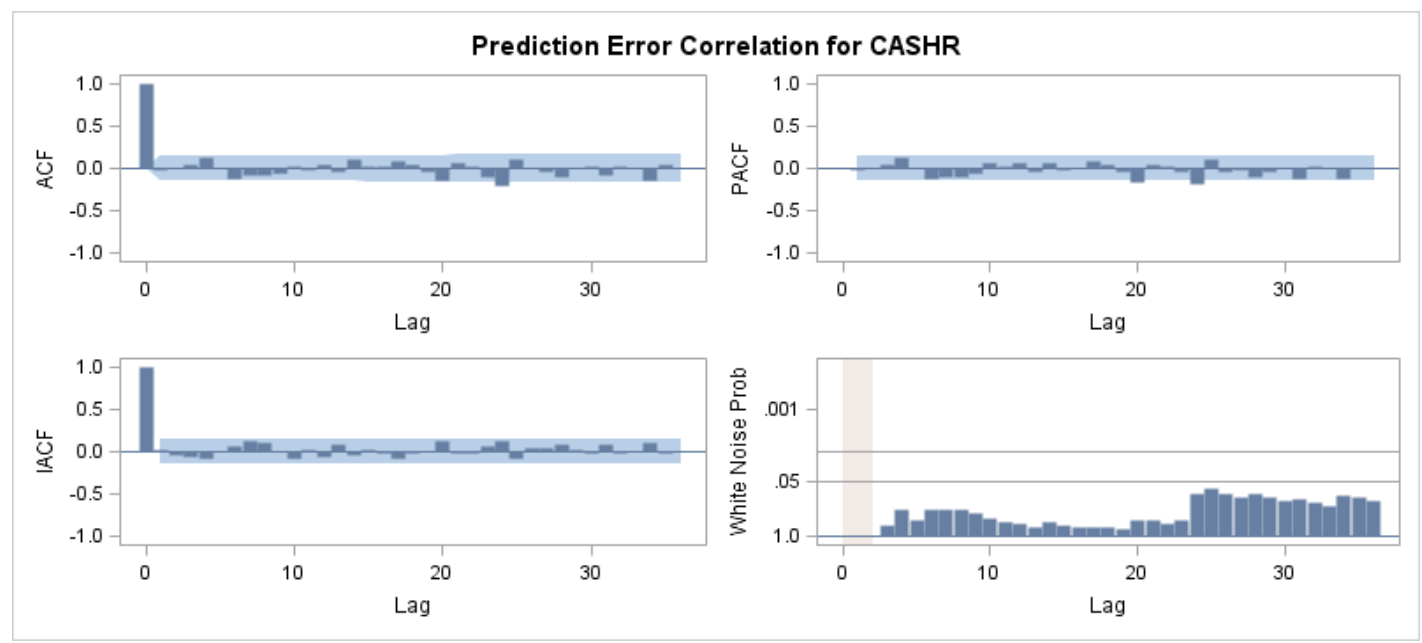

Figura 13 - Análise de Correlação dos Resíduos do Modelo ARMA (1, 10) 


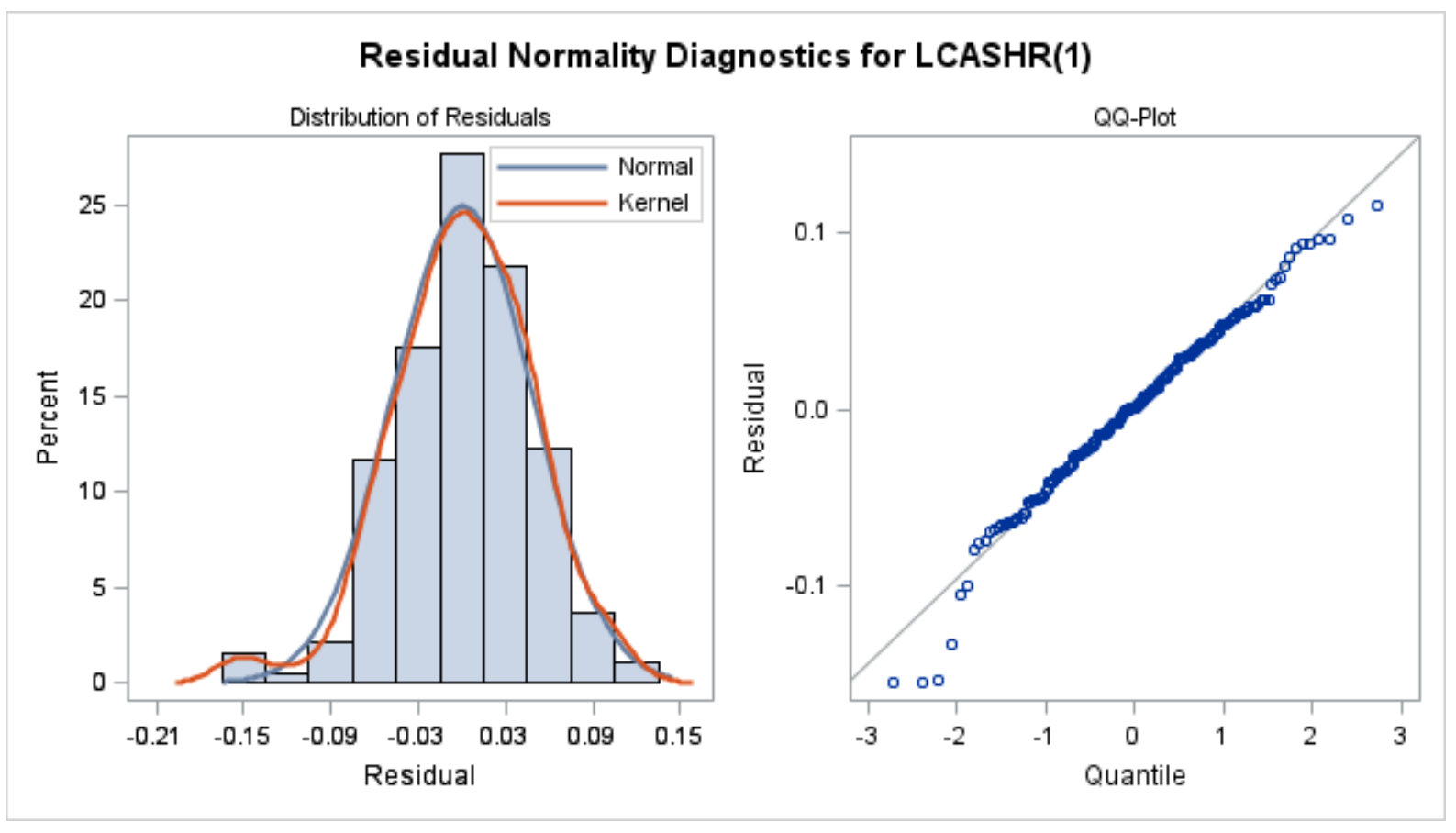

Figura 14 - Análise de Normalidade dos Resíduos do Modelo ARMA(1, 10)

Tabela 14 - Teste de Normalidade de Jarque-Bera - Modelo ARMA (1,10)

\begin{tabular}{cc}
\hline Estatística Jarque-Bera & Nível Descritivo \\
\hline 10,2284 & 0,006010 \\
\hline
\end{tabular}

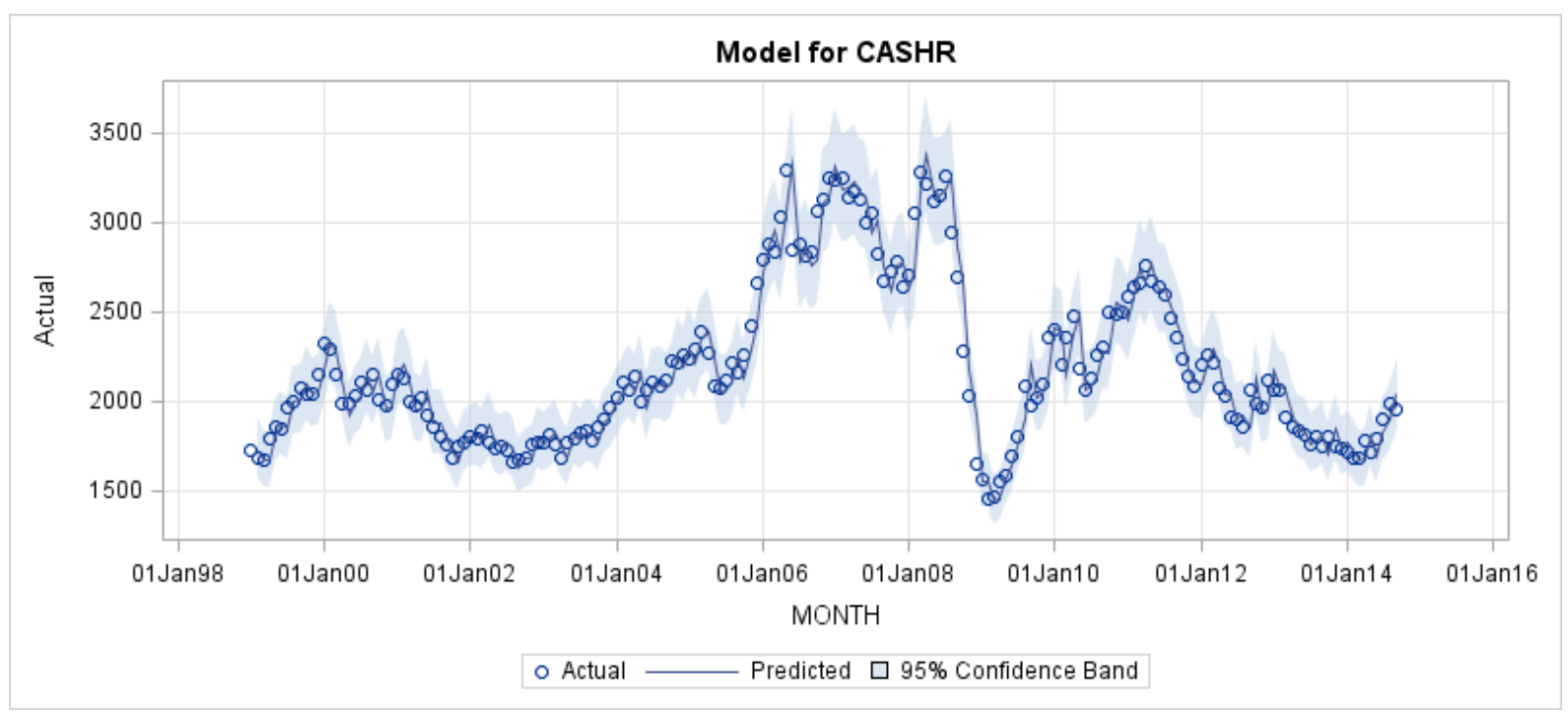

Figura 15 - Modelo ARMA $(1,10)$ Ajustado dentro da Amostra Completa 


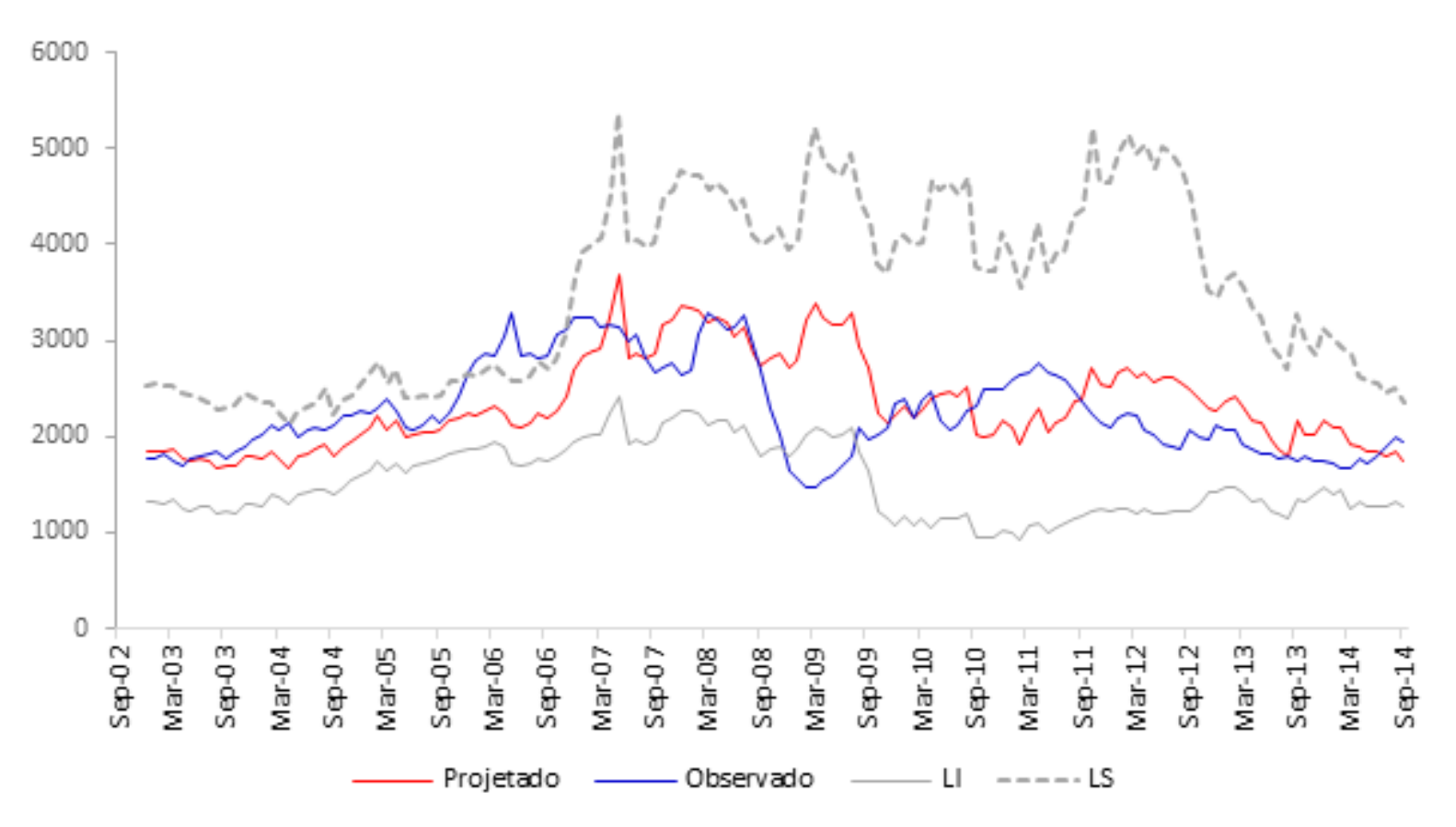

Figura 16 - Modelo ARMA(1, 10): Preços de Alumínio Observados e Projetados, 12 Meses à Frente Ponto a Ponto, Fora da Amostra (Dez/2002 - Set/2014, US\$/t)

\subsubsection{Modelos ARIMAX}

\subsubsection{Suposições para Especificação do Modelo ARIMAX}

As seguintes suposições são necessárias para a especificação do modelo ARIMAX (LUTKEPOHL, 2007; WOOLDRIDGE, 2010):

(a) Estacionariedade fraca das variáveis dependente e independentes;

(b) Exogeneidade fraca das variáveis independentes em relação à variável dependente. Desta forma, a esperança condicional do erro em relação às variáveis independentes é zero;

(c) Erro do modelo é um ruído branco, ou seja, segue distribuição normal, com média zero e variância constante. 
Não é condição necessária que as variáveis independentes Granger causem a variável dependente na especificação do modelo ARIMAX. No entanto, se incluirmos variáveis independentes, fracamente exógenas e estacionárias, que Granger causem a variável dependente, é possível melhorar a previsão da variável dependente.

Dessa forma, para a seleção das variáveis independentes do modelo ARIMAX, será realizado um quarto teste, que é o de causalidadade de Granger.

\subsubsection{Teste de Estacionariedade das Variáveis Independentes do Modelo ARIMAX}

O teste de estacionariedade ADF indica que, das 1.130 variáveis transformadas analisadas, 716 variáveis são estacionárias (Anexo A). As transformações realizadas foram variação percentual (1 a 6 defasagens - CHG), diferença (1 a 12 defasagens - DIF) e diferença do logaritmo natural (1 a 12 defasagens - DL).

A Tabela 14 apresenta as 716 variáveis independentes transformadas agrupadas por oito categorias: preços; produção industrial; produção de alumínio; consumo de alumínio; estoques de aluminio; câmbio; taxa de juros; mercado de ações. A segunda coluna agrupa as variáveis independentes (terceira coluna) em oito categorias.

As colunas quatro a seis da Tabela 14 mostram os números de modelos obtidos para cada tipo de transformação, onde CHG significa variação percentual de um a seis meses. Têm-se, portanto, seis modelos transformados em termos de variações percentuais. DIF significa as diferenças tomadas para cada variável, de uma a doze diferenças, em que a base é fixada. Por exemplo, modelo transformado com uma diferença seria obtido com a diferença do valor de fevereiro de 2013 a janeiro de 2013; modelo transformado com duas diferenças seria obtido com a diferença do valor da variável independente de março de 2013 a janeiro de 2013. Têmse, pois, 12 modelos transformados em termos de diferenças. DL significa logaritmo natural de variável independente tomada de uma a seis diferenças. Por exemplo, modelo transformado com uma diferença seria obtido pelo logaritmo natural da diferença do valor da variável independente de fevereiro de 2013 a janeiro de 2013; modelo transformado com duas diferenças seria obtido por meio do logarirmo natural da diferença do valor da variável 
independente de março de 2013 a janeiro de 2013, e assim sucessivamente. Têm-se, pois, seis modelos transformados por meio da diferença do logaritmo natural.

Tabela 15 - Variáveis Independentes Transformadas e Estacionárias

\begin{tabular}{cllrrrr}
\hline Item & Categoria & Variável & CHG & DIF & DL & Total \\
\hline 1 & Preços & M3R & 6 & 12 & 6 & 24 \\
2 & & BRENT & 6 & 12 & 6 & 24 \\
3 & CPI2013 & 6 & 12 & 6 & 24 \\
4 & NFINDEX & 6 & 12 & 6 & 24 \\
5 & & USPPINFM & 6 & 12 & 6 & 24 \\
\hline 6 & Produção industrial & USIP & 6 & 12 & 6 & 24 \\
7 & & USPMIP & 6 & 12 & 6 & 24 \\
8 & CHIP & 5 & 11 & 6 & 22 \\
9 & & EURIP & 6 & 12 & 6 & 24 \\
10 & & JAPIP & 6 & 12 & 6 & 24 \\
\hline 11 & Produção de alumínio primário & CAP & 5 & 11 & 6 & 22 \\
12 & & GLPROD & 6 & 12 & 6 & 24 \\
13 & & GLCAPUT & 6 & 12 & 6 & 24 \\
\hline 14 & Consumo de alumínio primário & GLCONS & 6 & 12 & 6 & 24 \\
15 & & EXCONS & 6 & 12 & 6 & 24 \\
\hline 16 & Estoques de alumínio primário & GLTOTDOC & 6 & 12 & 6 & 24 \\
17 & & GLREPDOC & 6 & 12 & 6 & 24 \\
18 & & EXREPDO & 6 & 12 & 6 & 24 \\
19 & & EXLMEDOC & 6 & 12 & 6 & 24 \\
20 & & GLSTOCK & 6 & 12 & 6 & 24 \\
21 & & GLREPSTO & 6 & 12 & 6 & 24 \\
22 & EXTOTSTO & 6 & 12 & 6 & 24 \\
23 & EXREPSTO & 6 & 12 & 6 & 24 \\
24 & & LMESTOCK & 6 & 12 & 6 & 24 \\
\hline 25 & Câmbio & FXUSD & 6 & 12 & 6 & 24 \\
26 & & FXEUREFF & 6 & 12 & 6 & 24 \\
27 & & FXJAP & 6 & 12 & 6 & 24 \\
\hline 28 & Taxa de juros & 6 & 12 & 6 & 24 \\
\hline 29 & Mercado de ações & 6 & 12 & 6 & 24 \\
30 & & SPSOR & 6 & 12 & 6 & 24 \\
\hline & TOTAL & 178 & 358 & 180 & 716 \\
\hline & & & & & & \\
\hline
\end{tabular}

\subsubsection{Teste de Exogeneidade Fraca das Varáveis Independentes para o Modelo} ARIMAX

O teste de exogeneidade fraca filtrou as 716 variáveis independentes e estacionárias iniciais para 212 variáveis, que são também fracamente exógenas. As Tabelas 16 e 18 mostram os resultados do teste de exogeneidade fraca para que essas 212 variáveis. A quarta coluna de cada tabela apresenta o nível descritivo da hipótese nula, em que a variável independente é 
fracamente exógena em relação à dependente (preço de alumínio). As Tabelas 16 e 18 mostram que o nível descritivo é maior que 5\%, confirmando a hipótese nula de exogeneidade fraca.

Tabela 16 - Teste de Exogeneidade Fraca para Variáveis Independentes Estacionárias

\begin{tabular}{|c|c|c|c|c|c|c|c|}
\hline Variável & DF & ChiSq & N. desc. & Variável & DF & ChiSq & N. desc. \\
\hline BRENT_CHG5 & 1 & 1.1771 & $27.8 \%$ & DLEXREPSTO_lag5 & 1 & 2.6654 & $10.3 \%$ \\
\hline BRENT_CHG6 & 1 & 0.2739 & $60.1 \%$ & DLEXREPSTO_lag6 & 1 & 2.0655 & $15.1 \%$ \\
\hline BRENT_dif11 & 1 & 2.7893 & $9.5 \%$ & DLEXTOTSTO_lag2 & 1 & 3.3796 & $6.6 \%$ \\
\hline BRENT_dif12 & 1 & 0.0762 & $78.3 \%$ & DLEXTOTSTO_lag3 & 1 & 0.2776 & $59.8 \%$ \\
\hline BRENT_dif6 & 1 & 1.5634 & $21.1 \%$ & DLEXTOTSTO_lag4 & 1 & 2.1057 & $14.7 \%$ \\
\hline CAP_CHG1 & 1 & 0.2022 & $65.3 \%$ & DLFXJAP & 1 & 3.3437 & $6.7 \%$ \\
\hline CAP_CHG2 & 1 & 1.8472 & $17.4 \%$ & DLFXJAP_lag4 & 1 & 0.4054 & $52.4 \%$ \\
\hline CAP_CHG3 & 1 & 1.1794 & $27.7 \%$ & DLFXJAP_lag5 & 1 & 0.3501 & $55.4 \%$ \\
\hline CAP_CHG4 & 1 & 1.0863 & $29.7 \%$ & DLFXJAP_lag6 & 1 & 1.4483 & $22.9 \%$ \\
\hline CAP_CHG5 & 1 & 0.2770 & $59.9 \%$ & DLGLREPDOC_lag5 & 1 & 2.9472 & $8.6 \%$ \\
\hline CAP_dif10 & 1 & 1.4905 & $22.2 \%$ & DLGLREPDOC_lag6 & 1 & 2.8311 & $9.2 \%$ \\
\hline CAP_dif11 & 1 & 0.3235 & $57.0 \%$ & DLGLREPSTO_lag2 & 1 & 3.6843 & $5.5 \%$ \\
\hline CAP_dif3 & 1 & 0.4818 & $48.8 \%$ & DLGLREPSTO_lag4 & 1 & 0.0249 & $87.5 \%$ \\
\hline CAP_dif4 & 1 & 2.2227 & $13.6 \%$ & DLGLREPSTO_lag5 & 1 & 0.1875 & $66.5 \%$ \\
\hline CAP_dif6 & 1 & 0.1477 & $70.1 \%$ & DLGLREPSTO_lag6 & 1 & 2.0481 & $15.2 \%$ \\
\hline CAP_dif7 & 1 & 0.2825 & $59.5 \%$ & DLGLSTOCK_lag2 & 1 & 3.1658 & $7.5 \%$ \\
\hline CAP_dif8 & 1 & 1.7172 & $19.0 \%$ & DLGLSTOCK_lag3 & 1 & 1.1204 & $29.0 \%$ \\
\hline CAP_dif9 & 1 & 1.3702 & $24.2 \%$ & DLGLSTOCK_lag4 & 1 & 2.9581 & $8.5 \%$ \\
\hline CHIP_CHG1 & 1 & 2.2803 & $13.1 \%$ & DLLIBOR_lag2 & 1 & 0.6185 & $43.2 \%$ \\
\hline CHIP_CHG2 & 1 & 1.9121 & $16.7 \%$ & DLLIBOR_lag3 & 1 & 3.3662 & $6.7 \%$ \\
\hline CHIP_CHG5 & 1 & 1.2399 & $26.5 \%$ & DLLIBOR_lag4 & 1 & 0.0510 & $82.1 \%$ \\
\hline CHIP_dif10 & 1 & 3.4416 & $6.4 \%$ & DLLIBOR_lag5 & 1 & 2.9087 & $8.8 \%$ \\
\hline CHIP_dif11 & 1 & 0.7401 & $39.0 \%$ & DLLIBOR_lag6 & 1 & 0.0110 & $91.7 \%$ \\
\hline CHIP_dif3 & 1 & 0.3360 & $56.2 \%$ & DLLMESTOCK_lag2 & 1 & 2.0160 & $15.6 \%$ \\
\hline CHIP_dif4 & 1 & 2.3068 & $12.9 \%$ & DLLMESTOCK_lag4 & 1 & 1.3237 & $25.0 \%$ \\
\hline CHIP_dif5 & 1 & 2.1219 & $14.5 \%$ & DLLMESTOCK_lag5 & 1 & 1.3815 & $24.0 \%$ \\
\hline CHIP_dif6 & 1 & 1.9177 & $16.6 \%$ & DLLMESTOCK_lag6 & 1 & 0.5043 & $47.8 \%$ \\
\hline CHIP_dif7 & 1 & 0.8523 & $35.6 \%$ & DLM3R & 1 & 0.1938 & $66.0 \%$ \\
\hline CHIP_dif8 & 1 & 0.2147 & $64.3 \%$ & DLM3R_lag4 & 1 & 1.3278 & $24.9 \%$ \\
\hline CHIP_dif9 & 1 & 2.2976 & $13.0 \%$ & DLM3R_lag5 & 1 & 0.0001 & $99.1 \%$ \\
\hline CPI2013_CHG5 & 1 & 2.9313 & $8.7 \%$ & DLM3R_lag6 & 1 & 2.9428 & $8.6 \%$ \\
\hline CPI2013_CHG6 & 1 & 1.2882 & $25.6 \%$ & DLNFINDEX_lag4 & 1 & 3.0332 & $8.2 \%$ \\
\hline CPI2013_dif11 & 1 & 3.2273 & $7.2 \%$ & DLUSPPINFM & 1 & 1.8240 & $17.7 \%$ \\
\hline CPI2013_dif12 & 1 & 1.1810 & $27.7 \%$ & DLUSPPINFM_lag4 & 1 & 0.3342 & $56.3 \%$ \\
\hline CPI2013_dif5 & 1 & 1.5023 & $22.0 \%$ & DLUSPPINFM_lag5 & 1 & 3.7670 & $5.2 \%$ \\
\hline CPI2013_dif6 & 1 & 0.9834 & $32.1 \%$ & DLUSPPINFM_lag6 & 1 & 0.1595 & $69.0 \%$ \\
\hline DJ_CHG2 & 1 & 3.6780 & $5.5 \%$ & EURIP_CHG1 & 1 & 0.0381 & $84.5 \%$ \\
\hline DJ_CHG3 & 1 & 2.5488 & $11.0 \%$ & EURIP_CHG2 & 1 & 0.1329 & $71.5 \%$ \\
\hline DJ_CHG4 & 1 & 3.7210 & $5.4 \%$ & EURIP_CHG3 & 1 & 0.2596 & $61.0 \%$ \\
\hline DJ_CHG6 & 1 & 1.4077 & $23.5 \%$ & EURIP_CHG4 & 1 & 0.4298 & $51.2 \%$ \\
\hline DJ_dif12 & 1 & 1.7806 & $18.2 \%$ & EURIP_CHG5 & 1 & 0.2622 & $60.9 \%$ \\
\hline DLCPI2013_lag3 & 1 & 2.0611 & $15.1 \%$ & EURIP_CHG6 & 1 & 0.0585 & $80.9 \%$ \\
\hline DLCPI2013_lag4 & 1 & 2.5104 & $11.3 \%$ & EURIP_dif10 & 1 & 0.6271 & $42.8 \%$ \\
\hline DLCPI2013_lag5 & 1 & 1.5212 & $21.7 \%$ & EURIP_dif11 & 1 & 0.3963 & $52.9 \%$ \\
\hline DLCPI2013_lag6 & 1 & 0.4477 & $50.3 \%$ & EURIP_dif12 & 1 & 0.0046 & $94.6 \%$ \\
\hline DLEXLMEDOC_lag2 & 1 & 2.2935 & $13.0 \%$ & EURIP_dif4 & 1 & 0.3203 & $57.1 \%$ \\
\hline DLEXLMEDOC_lag4 & 1 & 3.4335 & $6.4 \%$ & EURIP_dif5 & 1 & 0.7506 & $38.6 \%$ \\
\hline DLEXLMEDOC_lag5 & 1 & 1.2679 & $26.0 \%$ & EURIP_dif6 & 1 & 0.5094 & $47.5 \%$ \\
\hline DLEXLMEDOC_lag6 & 1 & 0.0011 & $97.3 \%$ & EURIP_dif7 & 1 & 0.1514 & $69.7 \%$ \\
\hline DLEXREPSTO_lag4 & 1 & 1.0837 & $29.8 \%$ & EURIP_dif8 & 1 & 0.2596 & $61.0 \%$ \\
\hline
\end{tabular}


Tabela 17 - Teste de Exogeneidade Fraca para Variáveis Independentes Estacionárias

\begin{tabular}{|c|c|c|c|}
\hline Variável & DF & ChiSq & N. desc. \\
\hline EURIP_dif9 & 1 & 0.4860 & $48.6 \%$ \\
\hline EXTOTSTO_CHG6 & 1 & 3.5892 & $5.8 \%$ \\
\hline EXTOTSTO_dif5 & 1 & 2.7619 & $9.7 \%$ \\
\hline FXEUREFF_CHG1 & 1 & 0.0433 & $83.5 \%$ \\
\hline FXEUREFF_CHG2 & 1 & 0.0184 & $89.2 \%$ \\
\hline FXEUREFF_CHG3 & 1 & 0.0497 & $82.4 \%$ \\
\hline FXEUREFF_CHG4 & 1 & 0.3010 & $58.3 \%$ \\
\hline FXEUREFF_CHG5 & 1 & 0.0652 & $79.8 \%$ \\
\hline FXEUREFF_CHG6 & 1 & 0.3391 & $56.0 \%$ \\
\hline FXEUREFF_dif10 & 1 & 0.4193 & $51.7 \%$ \\
\hline FXEUREFF_dif11 & 1 & 0.0723 & $78.8 \%$ \\
\hline FXEUREFF_dif12 & 1 & 0.2632 & $60.8 \%$ \\
\hline FXEUREFF_dif4 & 1 & 0.1560 & $69.3 \%$ \\
\hline FXEUREFF_dif5 & 1 & 1.3496 & $24.5 \%$ \\
\hline FXEUREFF_dif6 & 1 & 1.3662 & $24.2 \%$ \\
\hline FXEUREFF_dif7 & 1 & 0.0115 & $91.4 \%$ \\
\hline FXEUREFF_dif8 & 1 & 0.0021 & $96.4 \%$ \\
\hline FXEUREFF_dif9 & 1 & 0.1284 & $72.0 \%$ \\
\hline FXJAP_CHG5 & 1 & 1.3093 & $25.3 \%$ \\
\hline FXJAP_CHG6 & 1 & 1.5367 & $21.5 \%$ \\
\hline FXJAP_dif1 & 1 & 3.3309 & $6.8 \%$ \\
\hline FXJAP_dif11 & 1 & 1.0274 & $31.1 \%$ \\
\hline FXJAP_dif12 & 1 & 0.9127 & $33.9 \%$ \\
\hline FXJAP_dif2 & 1 & 3.3877 & $6.6 \%$ \\
\hline FXJAP_dif3 & 1 & 0.4507 & $50.2 \%$ \\
\hline FXJAP_dif4 & 1 & 3.7372 & $5.3 \%$ \\
\hline FXJAP_dif5 & 1 & 2.1072 & $14.7 \%$ \\
\hline FXJAP_dif6 & 1 & 2.4579 & $11.7 \%$ \\
\hline FXJAP_dif7 & 1 & 3.5621 & $5.9 \%$ \\
\hline FXUSD_CHG1 & 1 & 1.3485 & $24.6 \%$ \\
\hline FXUSD_CHG2 & 1 & 0.8193 & $36.5 \%$ \\
\hline FXUSD_CHG3 & 1 & 0.3573 & $55.0 \%$ \\
\hline FXUSD_CHG4 & 1 & 0.3719 & $54.2 \%$ \\
\hline FXUSD_CHG5 & 1 & 1.7430 & $18.7 \%$ \\
\hline FXUSD_CHG6 & 1 & 0.5972 & $44.0 \%$ \\
\hline FXUSD_dif10 & 1 & 0.1724 & $67.8 \%$ \\
\hline FXUSD_dif11 & 1 & 1.2632 & $26.1 \%$ \\
\hline FXUSD_dif12 & 1 & 0.3011 & $58.3 \%$ \\
\hline FXUSD_dif4 & 1 & 1.3472 & $24.6 \%$ \\
\hline FXUSD_dif5 & 1 & 0.0695 & $79.2 \%$ \\
\hline FXUSD_dif6 & 1 & 0.2017 & $65.3 \%$ \\
\hline FXUSD_dif7 & 1 & 0.7251 & $39.4 \%$ \\
\hline FXUSD_dif8 & 1 & 0.3380 & $56.1 \%$ \\
\hline FXUSD_dif9 & 1 & 0.0867 & $76.8 \%$ \\
\hline GLCAPUT_CHG6 & 1 & 2.9862 & $8.4 \%$ \\
\hline GLCAPUT_dif12 & 1 & 3.4598 & $6.3 \%$ \\
\hline GLCONS_CHG3 & 1 & 3.7504 & $5.3 \%$ \\
\hline GLCONS_CHG4 & 1 & 1.4182 & $23.4 \%$ \\
\hline GLCONS_CHG5 & 1 & 1.6836 & $19.4 \%$ \\
\hline GLCONS_dif10 & 1 & 2.5260 & $11.2 \%$ \\
\hline GLCONS_dif11 & 1 & 2.2727 & $13.2 \%$ \\
\hline GLCONS_dif5 & 1 & 2.5149 & $11.3 \%$ \\
\hline GLPROD_CHG1 & 1 & 3.2208 & $7.3 \%$ \\
\hline GLPROD_CHG2 & 1 & 0.4096 & $52.2 \%$ \\
\hline GLPROD_CHG3 & 1 & 1.9876 & $15.9 \%$ \\
\hline GLPROD_CHG4 & 1 & 0.6100 & $43.5 \%$ \\
\hline
\end{tabular}

\begin{tabular}{|c|c|c|c|}
\hline Variável & DF & ChiSq & N. desc. \\
\hline GLPROD_CHG5 & 1 & 0.4401 & $50.7 \%$ \\
\hline GLPROD_CHG6 & 1 & 1.7939 & $18.0 \%$ \\
\hline GLPROD_dif10 & 1 & 1.1148 & $29.1 \%$ \\
\hline GLPROD_dif11 & 1 & 0.8334 & $36.1 \%$ \\
\hline GLPROD_dif12 & 1 & 2.5674 & $10.9 \%$ \\
\hline GLPROD_dif3 & 1 & 3.7035 & $5.4 \%$ \\
\hline GLPROD_dif4 & 1 & 3.4110 & $6.5 \%$ \\
\hline GLPROD_dif5 & 1 & 1.4099 & $23.5 \%$ \\
\hline GLPROD_dif8 & 1 & 1.3410 & $24.7 \%$ \\
\hline GLPROD_dif9 & 1 & 3.3800 & $6.6 \%$ \\
\hline GLSTOCK_CHG6 & 1 & 3.5993 & $5.8 \%$ \\
\hline GLSTOCK_dif4 & 1 & 2.1130 & $14.6 \%$ \\
\hline GLSTOCK_dif5 & 1 & 0.9783 & $32.3 \%$ \\
\hline GLSTOCK_dif6 & 1 & 2.3346 & $12.7 \%$ \\
\hline JAPIP_CHG5 & 1 & 0.8866 & $34.6 \%$ \\
\hline JAPIP_dif11 & 1 & 1.8318 & $17.6 \%$ \\
\hline LIBOR_CHG1 & 1 & 3.1556 & $7.6 \%$ \\
\hline LIBOR_CHG4 & 1 & 2.9559 & $8.6 \%$ \\
\hline LIBOR_CHG5 & 1 & 2.2401 & $13.4 \%$ \\
\hline LIBOR_dif10 & 1 & 3.4063 & $6.5 \%$ \\
\hline LIBOR_dif11 & 1 & 0.4021 & $52.6 \%$ \\
\hline LIBOR_dif12 & 1 & 0.3756 & $54.0 \%$ \\
\hline LIBOR_dif3 & 1 & 3.2770 & $7.0 \%$ \\
\hline LIBOR_dif8 & 1 & 2.1930 & $13.9 \%$ \\
\hline LIBOR_dif9 & 1 & 2.4041 & $12.1 \%$ \\
\hline M3R_CHG3 & 1 & 2.1541 & $14.2 \%$ \\
\hline M3R_CHG4 & 1 & 2.3066 & $12.9 \%$ \\
\hline M3R_CHG5 & 1 & 2.4502 & $11.8 \%$ \\
\hline M3R_CHG6 & 1 & 2.1142 & $14.6 \%$ \\
\hline M3R_dif1 & 1 & 2.2149 & $13.7 \%$ \\
\hline M3R_dif2 & 1 & 2.0355 & $15.4 \%$ \\
\hline M3R_dif9 & 1 & 3.4683 & $6.3 \%$ \\
\hline NFINDEX_CHG1 & 1 & 1.4568 & $22.7 \%$ \\
\hline NFINDEX_CHG2 & 1 & 0.0834 & $77.3 \%$ \\
\hline NFINDEX_CHG3 & 1 & 1.3874 & $23.9 \%$ \\
\hline NFINDEX_CHG4 & 1 & 0.5550 & $45.6 \%$ \\
\hline NFINDEX_CHG5 & 1 & 1.6369 & $20.1 \%$ \\
\hline NFINDEX_CHG6 & 1 & 0.0060 & $93.8 \%$ \\
\hline NFINDEX_dif10 & 1 & 0.6618 & $41.6 \%$ \\
\hline NFINDEX_dif11 & 1 & 1.3567 & $24.4 \%$ \\
\hline NFINDEX_dif12 & 1 & 0.0018 & $96.7 \%$ \\
\hline NFINDEX_dif4 & 1 & 3.2985 & $6.9 \%$ \\
\hline NFINDEX_dif7 & 1 & 1.8253 & $17.7 \%$ \\
\hline NFINDEX_dif8 & 1 & 0.1945 & $65.9 \%$ \\
\hline NFINDEX_dif9 & 1 & 1.2134 & $27.1 \%$ \\
\hline SP500_CHG2 & 1 & 3.7656 & $5.2 \%$ \\
\hline SP500_CHG3 & 1 & 2.1790 & $14.0 \%$ \\
\hline SP500_CHG4 & 1 & 2.8501 & $9.1 \%$ \\
\hline SP500_CHG6 & 1 & 1.4675 & $22.6 \%$ \\
\hline SP500_dif10 & 1 & 3.1732 & $7.5 \%$ \\
\hline SP500_dif11 & 1 & 3.6570 & $5.6 \%$ \\
\hline SP500_dif12 & 1 & 1.9229 & $16.6 \%$ \\
\hline SP500_dif4 & 1 & 3.1451 & $7.6 \%$ \\
\hline SP500_dif5 & 1 & 3.0540 & $8.1 \%$ \\
\hline USPPINFM_CHG6 & 1 & 2.5845 & $10.8 \%$ \\
\hline USPPINFM_dif1 & 1 & 3.1232 & $7.7 \%$ \\
\hline
\end{tabular}




\subsubsection{Teste de Causalidade de Granger}

O teste de causalidade de Granger filtrou as 212 variáveis independentes, estacionárias e fracamente exógenas para 19 variáveis. A Tabela 18 mostra que essas 19 variáveis Granger causam o preço do alumínio, mas não vice-versa.

A quarta coluna da Tabela 18 apresenta o nível descritivo do teste de causalidade de Granger para a hipótese nula de que a variável independente não Granger causa a dependente (preço de alumínio). Deseja-se, portanto, rejeitar essa hipótese nula. Dessa forma, selecionaram-se as variáveis independentes tais que os níveis descritivos da quarta coluna sejam menores que 0,05.

A quinta coluna da Tabela 18 apresenta o nível descritivo do teste de causalidade de Granger para a hipótese nula de que a variável dependente (preço de alumínio) não Granger causa a variável independente. Deseja-se, portanto, aceitar essa hipótese nula. Dessa forma, selecionaram-se as variáveis independentes tais que os níveis descritivos da quinta coluna sejam maiores que 0,05 .

Portanto, com base nos testes de estacionariedade, exogeneidade fraca, e de causalidade de Granger, anteriormente descritos, foram selecionadas 13 variáveis independentes para o ajuste do modelo ARMAX, conforme Tabela 18.

Tabela 18 - Teste de Causalide de Granger para Variáveis Independentes Fracamente Estacionárias e Exógenas

\begin{tabular}{|c|c|c|c|c|c|}
\hline Categoria & Variável & Var. Transformada & Transformação & $\begin{array}{l}\text { Var. } \longrightarrow \text { Preço Alumínio } \\
\text { (Nível descr. rejeitar } \mathrm{H}_{0}=\text { Não Causa) }\end{array}$ & $\begin{array}{l}\text { Preço Alumínio } \leftrightarrow \longrightarrow \text { Var. } \\
\text { (Nivel descr. rejeitar } H_{0}=\text { Não Causa) }\end{array}$ \\
\hline \multirow[t]{6}{*}{ Preços } & M3R & DLM3R_lag4 & Diferença do log defasado de 4 meses & 0,0544 & 0,2817 \\
\hline & BRENT & BRENT_DIF12 & Diferença de 12 meses & 0,0016 & 0,2251 \\
\hline & CPI2013 & DLCPI2013_lag3 & Diferença do log defasado de 3 meses & 0,0255 & 0,6071 \\
\hline & NFINDEX & NFINDEX_DIF4 & Diferença de 4 meses & 0,0020 & 0,1067 \\
\hline & NFINDEX & NFINDEX_CHG5 & Variação percentual de 5 meses & 0,0146 & 0,7926 \\
\hline & USPMIP & DLUSPMIP_lag5 & Diferença do log defasado de 5 meses & 0,0718 & 0,7547 \\
\hline \multirow[t]{2}{*}{ Produção industrial } & CHIP & CHIP_CHG1 & Diferença de 1 mês & 0,0538 & 0,1587 \\
\hline & JAPIP & JAPIP_CHG5 & Var. percentual de 5 meses & 0,0847 & 0,2707 \\
\hline \multirow[t]{2}{*}{ Estoques de alumínio primário } & GLSTOCK & GLSTOCK_CHG6 & Var. percentual de 6 meses & 0,0480 & 0,1304 \\
\hline & EXTOTSTO & EXTOTSTO_CHG6 & Var. percentual de 6 meses & 0,0311 & 0,1408 \\
\hline Câmbio & FXUSD & FXUSD_dif4 & Diferença de 4 meses & 0,0915 & 0,7700 \\
\hline \multirow[t]{2}{*}{ Mercado de ações } & DJ & DJ_dif1 & Diferença de 1 mês & 0,0538 & 0,1587 \\
\hline & SP500 & SP500_dif1 & Diferença de 1 mês & 0,0226 & 0,2097 \\
\hline
\end{tabular}




\subsubsection{Modelos ARIMAX Propostos}

\subsection{Modelo ARIMAX 1}

Foram estimados possíveis modelos usando combinações das variáveis selecionadas da Tabela 18, com base na amostra completa (janeiro/1999 a setembro/2014). Selecionou-se o modelo com o menor critério de informação de Schwartz.

As variáveis independentes para o modelo selecionado foram:

- $\quad$ BRENT_dif12: diferença de 12 defasagens do preço de petróleo tipo Brent

- $\quad$ NFINDEX_dif4: diferença de 4 defasagens do índice de metais não-ferrosos

A Tabela 19 mostra os resultados na estimação da amostra completa (janeiro/1999 a setembro/2014) e a Tabela 20 apresenta estatísticas de ajuste do modelo. Todos os parâmetros foram significativos a $5 \%$, incluindo um componente $\mathrm{AR}(1)$.

Tabela 19 - Estimação do Modelo ARIMAX 1

\begin{tabular}{cccccc}
\hline Variável & Parâmetro & Estimativa & Erro-Padrão & $\mathrm{t}$ & Nível Descritivo \\
\hline NFINDEX_dif4 & Var. Ind. & 0,0056 & 0,0010 & 5,47 & $<0,0001$ \\
\hline BRENT_dif12 & Var. Ind. & 0,0005 & 0,0002 & 3,24 & 0,0012 \\
\hline CASHR & AR(1) & 0,15253 & 0,0749 & 2,04 & 0,0418 \\
\hline
\end{tabular}

Tabela 20 - Estatísticas de Ajuste do Modelo ARIMAX 1

\begin{tabular}{cc}
\hline Estatística & Valor \\
\hline AIC & $1.664,6904$ \\
SBC & $1.674,2188$ \\
RMSE & 108,3716 \\
MAPE & 3,6650 \\
MAE & 81,9496 \\
R-Quadrado & 0,9439 \\
R-Quadrado Ajustado & 0,9433 \\
\hline
\end{tabular}


A análise de correlação dos resíduos (Figura 17) mostra que não há autocorrelação diferente de zero, indicando adequação do modelo. A análise de normalidade (Figura 18) indica que a distribuição dos resíduos aproxima-se de uma distribuição normal. A Figura 19 aponta que os resultados estimados dentro da amostra estão dentro do intervalo de confiança de $95 \%$. A Tabela 21 apresenta o resultado do teste de normalidade de Jarque-Bera para a janela de 36 meses de janeiro/2010 a dezembro/2013, com um nível descritivo menor que 0,05 e, portanto, confirmando que os resíduos seguem uma distribuição normal.

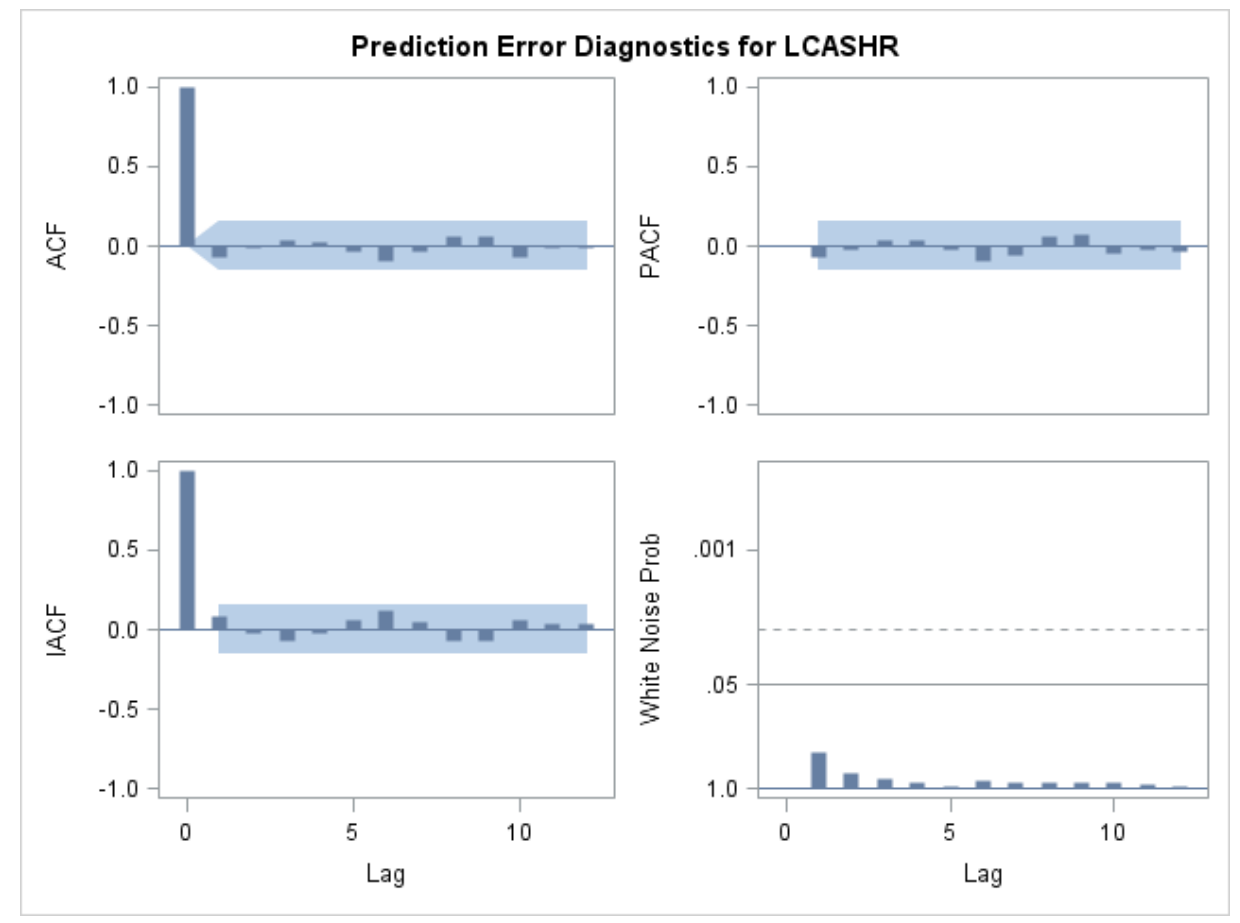

Figura 17 - Análise de Correlação dos Resíduos do Modelo ARIMAX 1
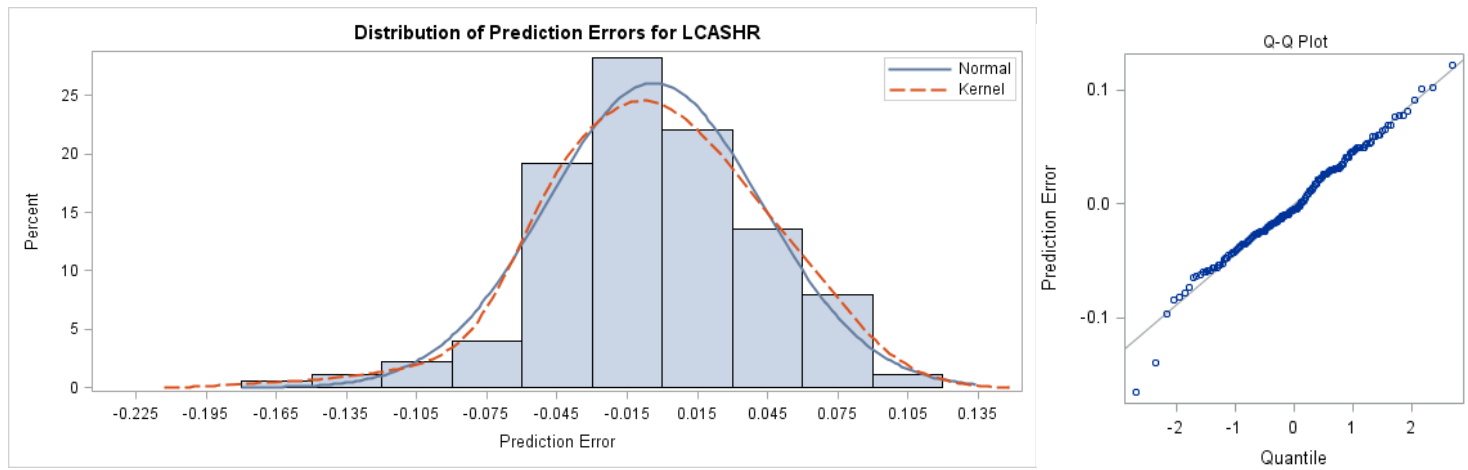

Figura 18 - Análise de Normalidade dos Resíduos do Modelo ARIMAX 1 
Tabela 21 - Teste de Normalidade de Jarque-Bera - Modelo ARMAX 1

\begin{tabular}{cc}
\hline Estatística Jarque-Bera & Nível Descritivo \\
\hline 6,8169 & 0,033092 \\
\hline
\end{tabular}

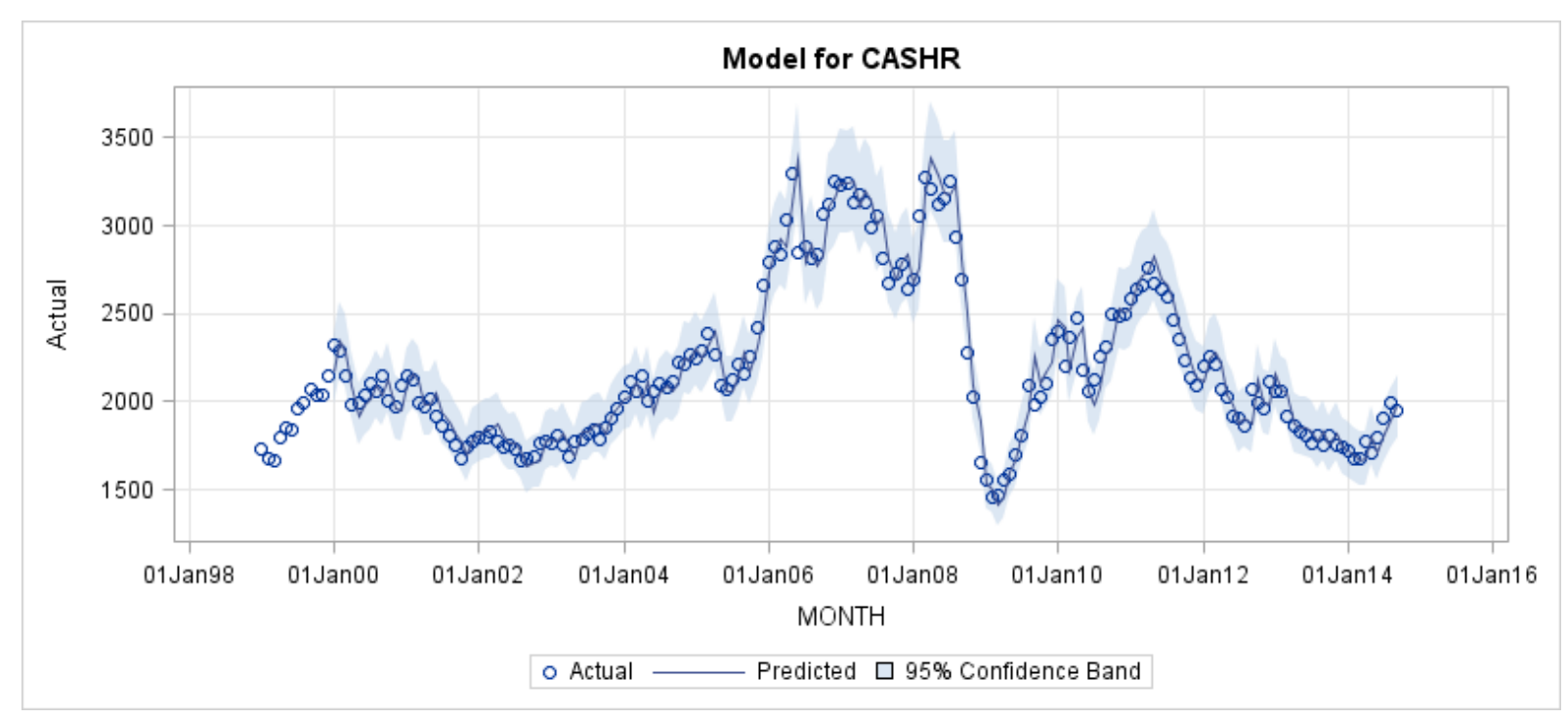

Figura 19 - Modelo ARIMAX 1 Ajustado dentro da Amostra Completa

No modelo ARIMAX, é necessário fornecer, para cada um dos 142 pontos, fora da amostra, a projeção 12 meses à frente das variáveis independentes. Para o modelo ARIMAX 1, essas variáveis são BRENT_dif12 (diferença de 12 meses do preço do petróleo tipo Brent) e NFINDEX_dif4 (diferença de 4 meses do preço do índice de preço de metais não-ferrosos nos Estados Unidos).

Para a projeção de 12 meses de BRENT_dif12 e NFINDEX_dif4, foi selecionado o método com menor MAPE, entre os seguintes abaixo. A seleção do método muda para cada uma das 142 projeções fora da amostra (conforme a janela móvel de 36 meses) e é realizada automaticamente por uma rotina no Forecast Server do SAS 9.4.

- $\quad$ Alisamento exponencial de Winters - aditivo

- $\quad$ Alisamento exponencial de Winters - multiplicativo

- Alisamento sazonal

- Alisamento linear 
Os preços projetados, 12 meses à frente por meio da janela móvel de estimação de 36 meses, estão apresentados na Figura 20, juntamente com o valores observados.

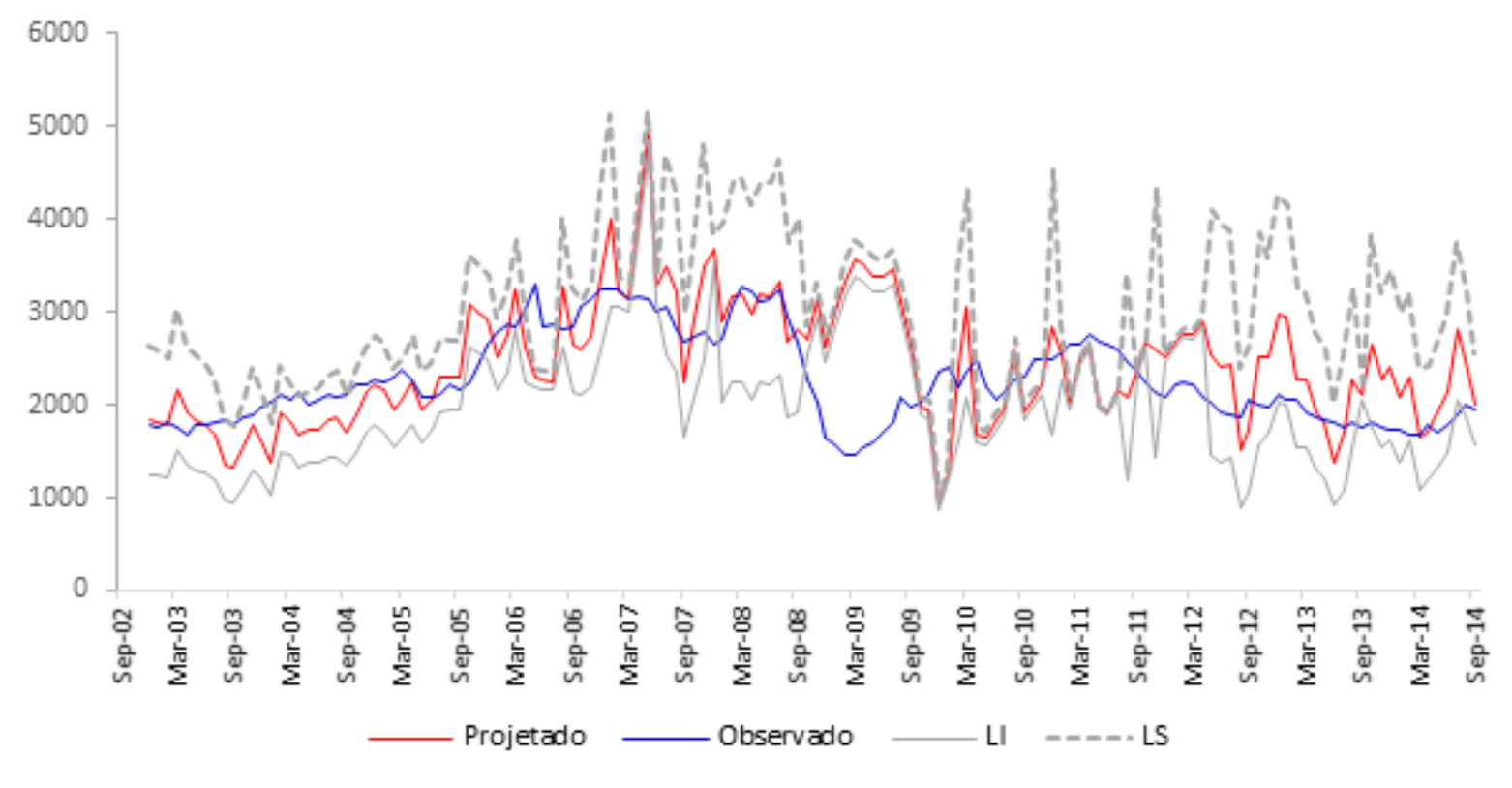

Figura 20 - Modelo ARIMAX 1: Preços Observados e Projetados, (Dez/2002 - Set/2014, US\$/t)

\subsection{Modelo ARIMAX 2}

Para o segundo modelo ARIMAX proposto, foram utilizadas as 2 variáveis independentes abaixo:

- $\quad$ DLM3R: diferença do log do preço futuro de alumínio de 3 meses

- $\quad$ DLGLSTOCK: diferença do log do estoque mundial de alumínio

Além de atenderem às suposições do modelo ARIMAX, essas variáveis caracterizam-se por aspectos relevantes: (i) há uma forte correlação positiva entre o preço à vista e futuro de 3 meses (99\%); (ii) no mercado global de alumínio, dias de estoque reportados pela indústria apresenta uma correlação negativa de 52\% com o preço à vista. Após muitas modelagens, a 
variável que, entre todas as 216 variáveis independentes associadas a estoques (tais como níveis de estoques globais ou por região; estoques da LME ou em trânsito; dias de estoque e suas transformadas), apresentou melhores resultados nas estimações da janela móvel de 3 anos foi a diferença do logaritmo natural dos estoques mundiais de alumínio.

A Figura 21 apresenta a inversão de comportamento entre estoques e preço de alumínio. Vale ressaltar que na crise global financeira de 2008/2009, os estoques de alumínio subiram significativamente, sendo uma das razões fundamentais da queda abrupta de preços observada no início de 2009.

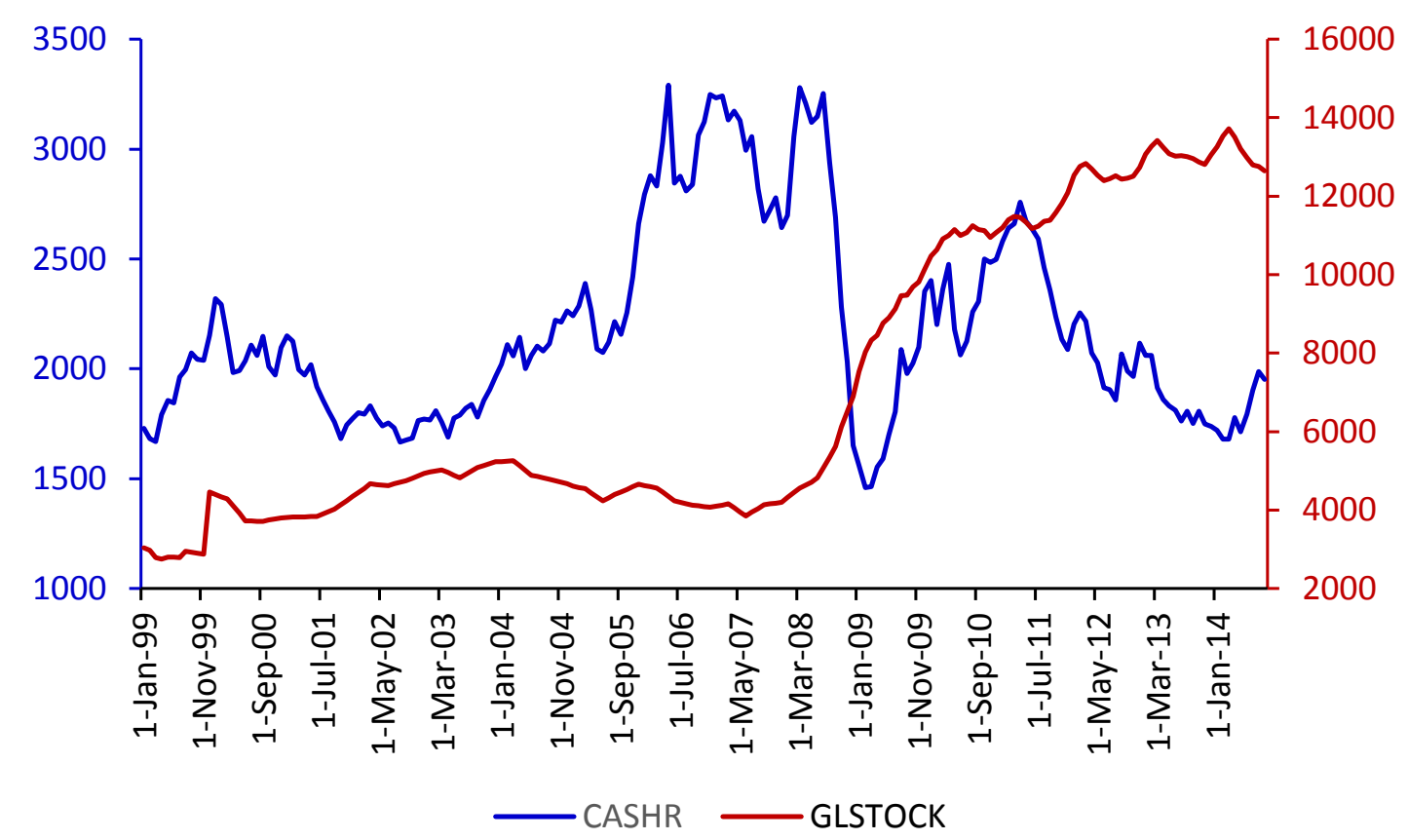

Figura 21 - Evolução do preço à vista (US\$/t) e estoque mundial de alumínio (kt), Jan/1999 - Set/2014

A Tabela 22 mostra os resultados para a estimação mais recente (outubro/2010-setembro/2013) associada à última projeção fora da amostra, considerando a brusca inflexão no aumento de estoques com a crise de 2008/2009 (Figura 21). A Tabela 23 apresenta estatísticas de ajuste do modelo. Todos os parâmetros foram significativos a 10\%, sem necessidade de componente ARMA. Vale notar que o RMSE (Tabela 23) foi de apenas 10,2572, e as outras métricas de erro de projeção como o MAPE e MAE também foram pequenas. 
Tabela 22 - Estimação do Modelo ARIMAX 2

\begin{tabular}{ccccc}
\hline Variável & Estimativa & Erro-Padrão & $\mathrm{t}$ & $\operatorname{Pr}>|t|$ \\
\hline DLM3R & 1,0160 & 0,0205 & 49,56 & 0,0001 \\
\hline DLGLSTOCK & $-0,0988$ & 0,0589 & $-1,68$ & 0,0938 \\
\hline
\end{tabular}

Tabela 23 - Estatísticas de Ajuste do Modelo ARIMAX 2

\begin{tabular}{cc}
\hline Estatística & Valor \\
\hline AIC & 166,9586 \\
SBC & 170,0694 \\
RMSE & 10,2572 \\
MAPE & 0,3449 \\
MAE & 7,5960 \\
R-Quadrado & 0,9988 \\
R-Quadrado Ajustado & 0,9988 \\
\hline
\end{tabular}

A análise de correlação dos resíduos (Figura 22) mostra que não há autocorrelação diferente de zero, indicando adequação do modelo. A análise de normalidade (Figura 23) indica que a distribuição dos resíduos aproximam-se de uma distribuição normal.

A Tabela 24 apresenta o resultado do teste de normalidade de Jarque-Bera para toda amostra, com um nível descritivo inferior a 0,05 e, portanto, confirmando a normalidade dos resíduos. A Figura 24 aponta que os resultados estimados dentro da amostra estão dentro do intervalo de confiança de $95 \%$. 

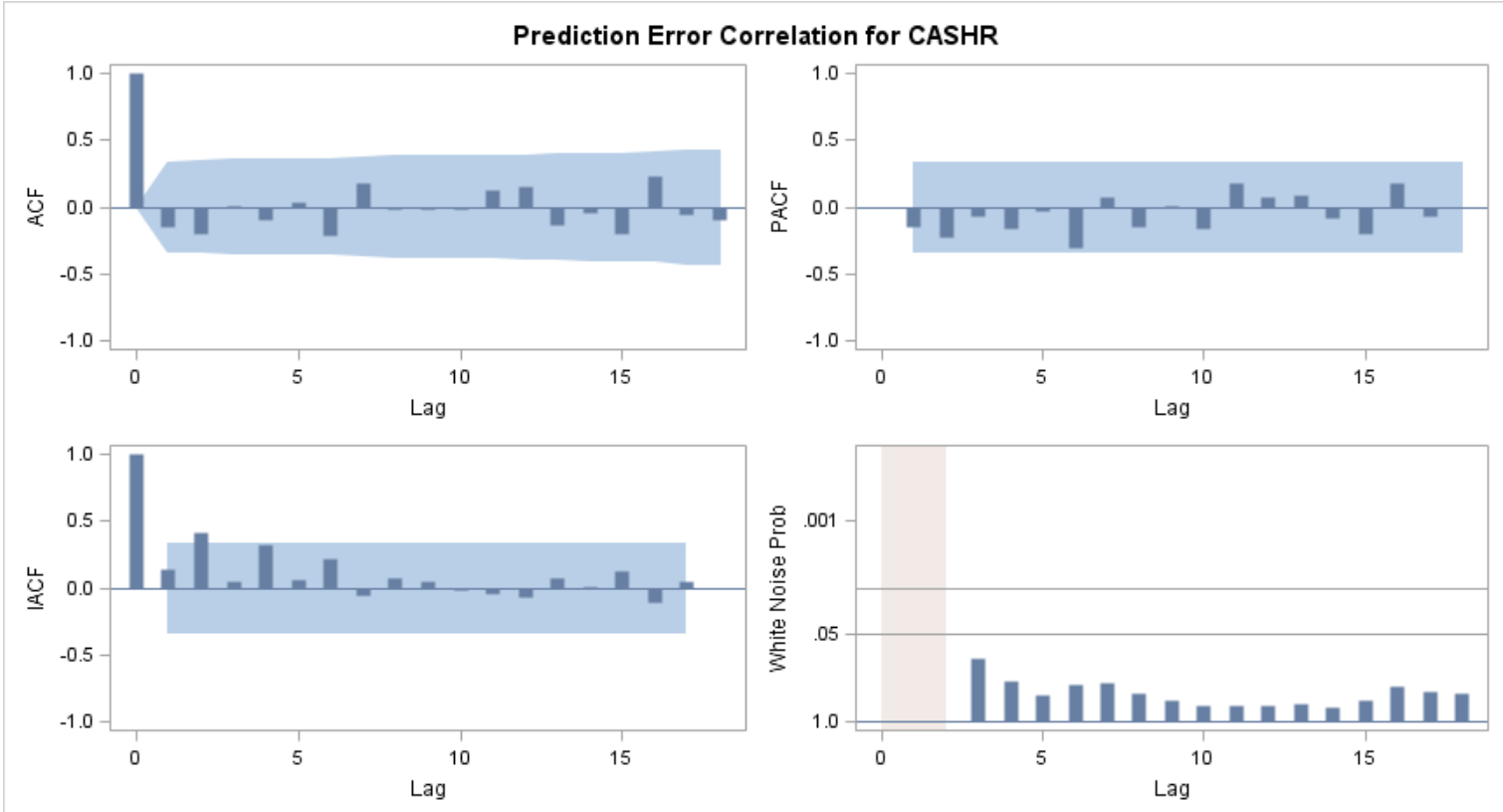

Figura 22 - Análise de Correlação dos Resíduos do Modelo ARIMAX 2

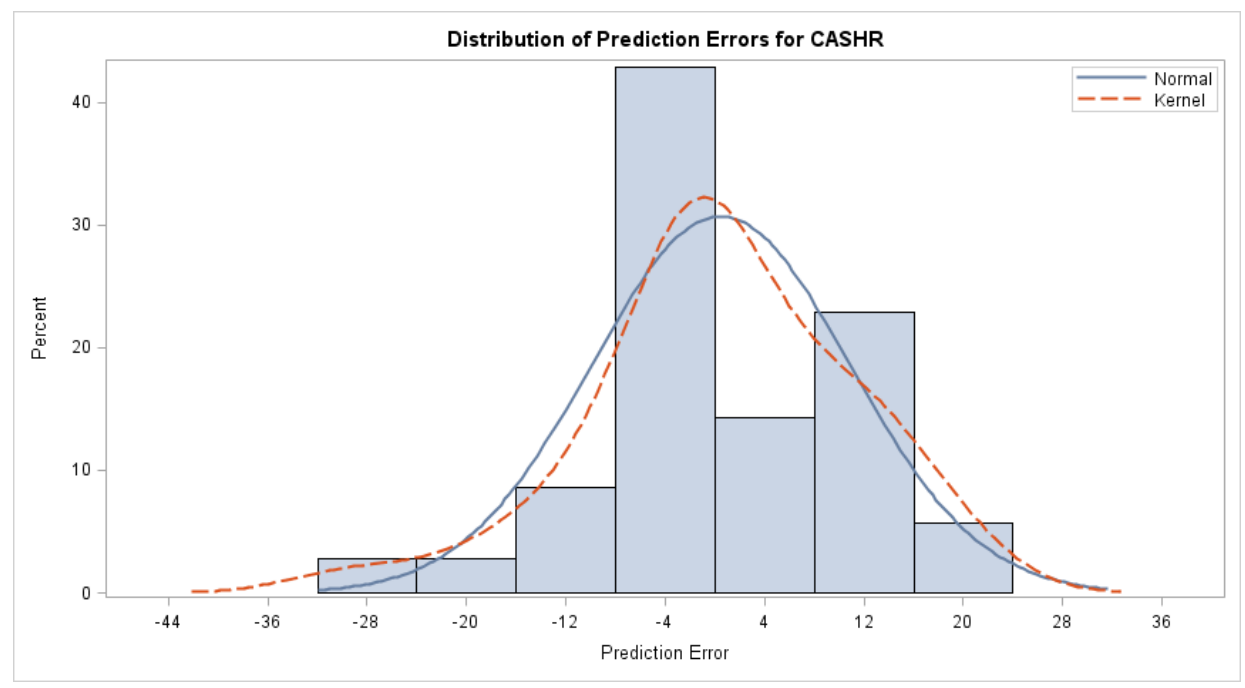

Figura 23 - Análise de Normalidade dos Resíduos do Modelo ARIMAX 2

Tabela 24 - Teste de Normalidade de Jarque-Bera - Modelo ARMAX 2

\begin{tabular}{cc}
\hline Estatística Jarque-Bera & Nível Descritivo \\
\hline 149,0791 & $<0,00001$ \\
\hline
\end{tabular}




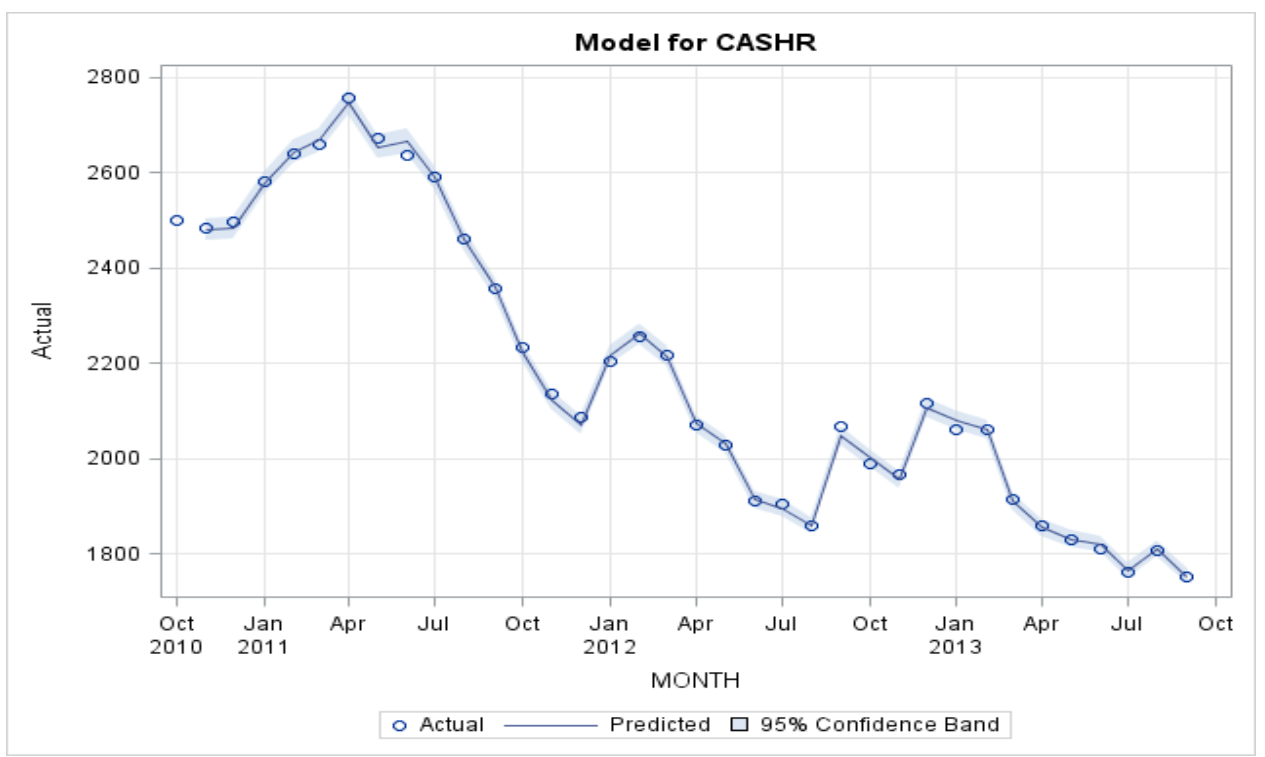

Figura 24 - Modelo ARIMAX 2 Ajustado para a Estimação mais Recente (out/2010-set/2013)

Como no modelo ARIMAX 1, para a projeção de 12 meses de M3R e GLSTOCK, foi selecionado o método com menor MAPE, entre os seguintes abaixo. A seleção do método muda para cada uma das 142 projeções fora da amostra (conforme a janela móvel de 36 meses) e é realizada automaticamente por uma rotina no Forecast Server do SAS 9.4.

- $\quad$ Alisamento exponencial de Winters - aditivo

- $\quad$ Alisamento exponencial de Winters - multiplicativo

- $\quad$ Alisamento sazonal

- $\quad$ Alisamento linear

Os preços projetados, 12 meses à frente por meio da janela móvel de estimação de 36 meses, estão apresentados na Figura 25, juntamente com o valores observados. 


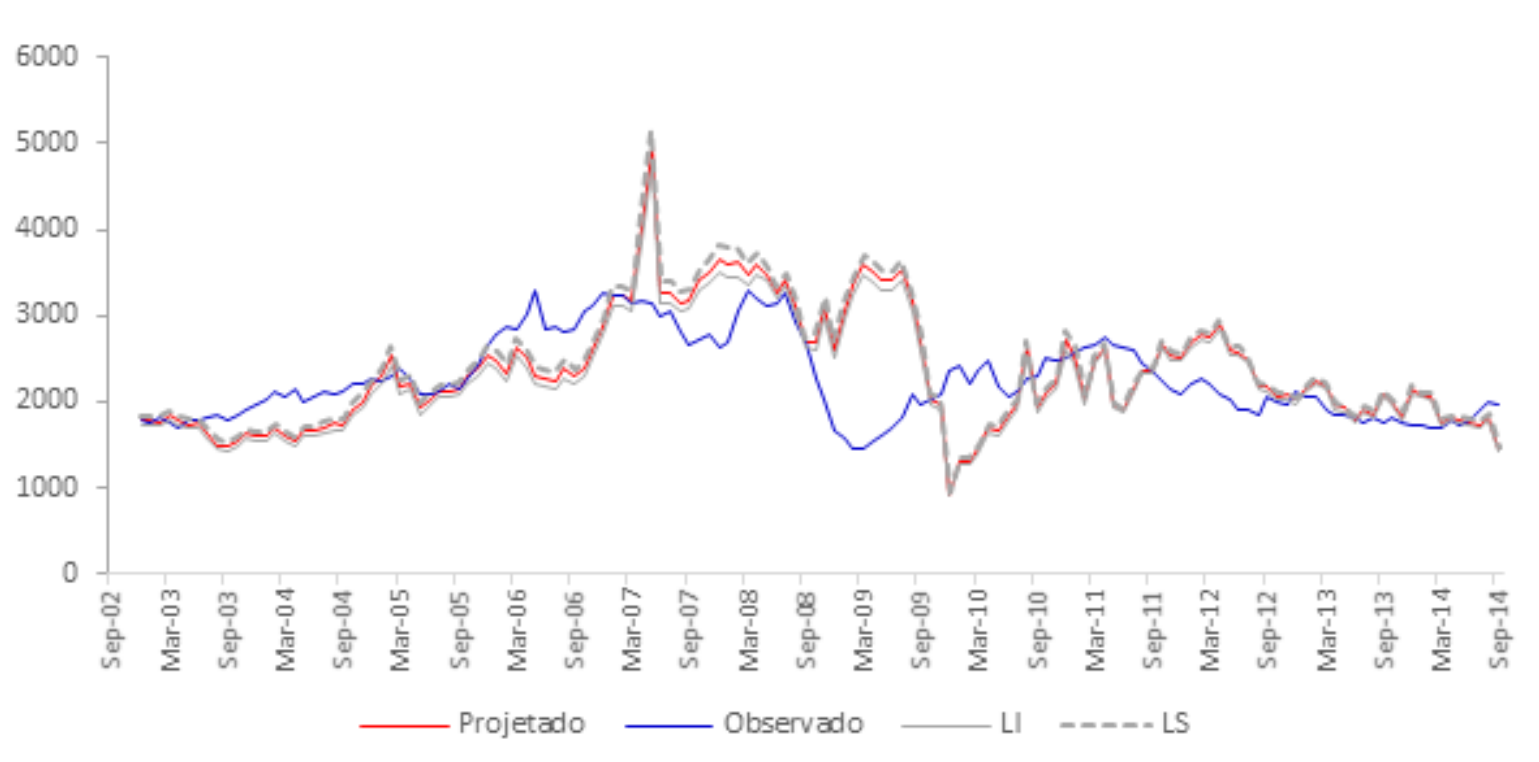

Figura 25 - Modelo AR1MAX 2: Preços Observados e Projetados (Dez/2002 - Set/2014, US\$/t)

\subsubsection{Modelo Estrutural}

Utilizou-se o logaritmo natural do preço à vista do alumínio como variável dependente para evitar valores projetados negativos. O modelo estrutural incluiu componentes de nível e ciclo, pois na amostra completa o componente de tendência não foi significativo.

A Tabela 25 apresenta os resultados do ajuste do modelo estrutural para a amostra completa (janeiro/1999 a setembro/2014). Os componentes de nível e ciclo foram significativos a um nível descritivo de 5\%. A Tabela 26 apresenta estatísticas de ajuste do modelo.

Tabela 25 - Estimação do Modelo Estrutural

\begin{tabular}{cccccc}
\hline Componente & Parâmetro & Estimativa & Erro-Padrão & $\mathrm{t}$ & $\operatorname{Pr}>|t|$ \\
\hline Nível & Variância & 0,0017551 & 0,0003098 & 5,67 & $<0,0001$ \\
\hline Ciclo & Variância & 0,0003056 & 0,0001544 & 1,98 & 0,0478 \\
\hline
\end{tabular}


Tabela 26 - Estatísticas de Ajuste do Modelo Estrutural

\begin{tabular}{cc}
\hline Estatística & Valor \\
\hline AIC & $1.784,4243$ \\
SBC & $1.797,3701$ \\
RMSE & 112,6778 \\
MAPE & 3,7978 \\
MAE & 84,9062 \\
R-Quadrado & 0,9375 \\
R-Quadrado Ajustado & 0,9365 \\
\hline
\end{tabular}

A análise de correlação dos resíduos (Figura 26) mostra que não há autocorrelação diferente de zero até a vigésima defasagem. A análise de normalidade (Figura 27) indica que a distribuição dos resíduos aproxima-se de uma distribuição normal. A Tabela 27 apresenta o resultado do teste de normalidade de Jarque-Bera com um nível descritivo inferior 0,05 e, portanto, confirmando a normalidade dos resíduos. A Figura 28 mostra que os resultados estimados dentro da amostra completa estão dentro do intervalo de confiança de $95 \%$.

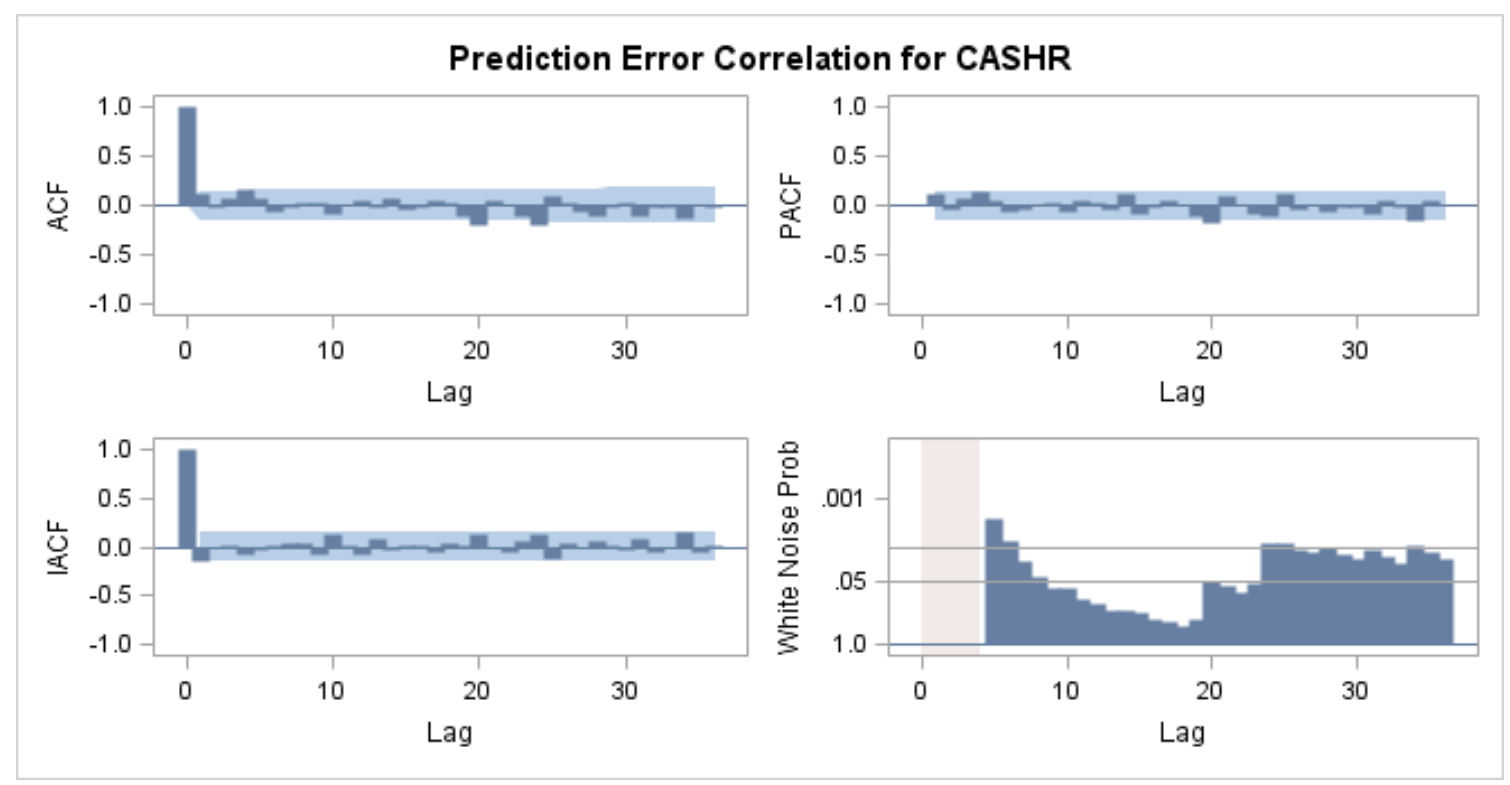

Figura 26 - Análise de Correlação dos Resíduos do Modelo Estrutural 


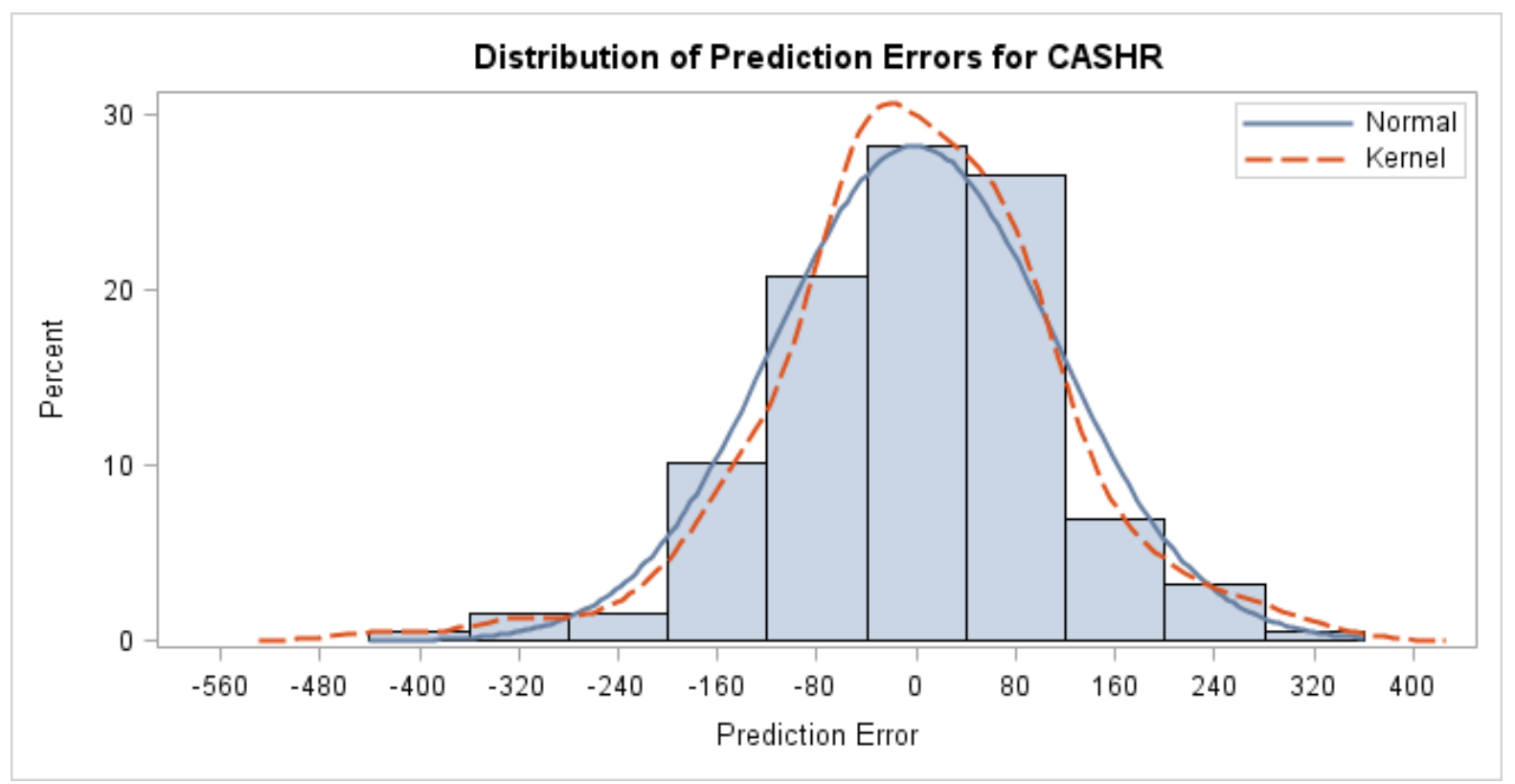

Figura 27 - Análise de Normalidade dos Resíduos do Modelo Estrutural

Tabela 27 - Teste de Normalidade de Jarque-Bera - Modelo Estrutural

\begin{tabular}{cc}
\hline Estatística Jarque-Bera & Nível Descritivo \\
\hline 8,52080 & 0,014117 \\
\hline
\end{tabular}

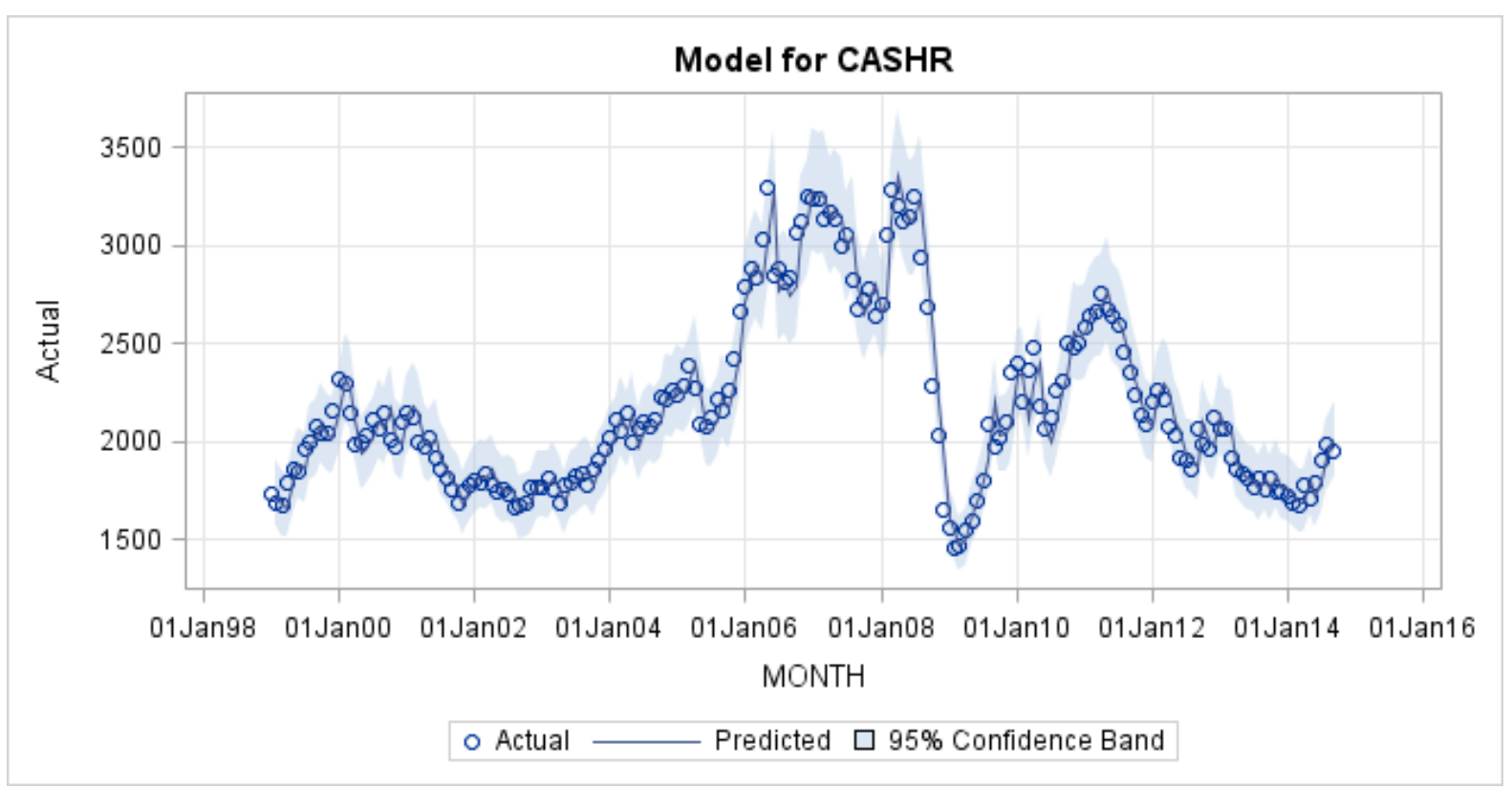

Figura 28 - Modelo Estrutural Ajustado dentro da Amostra Completa 
Os preços projetados, 12 meses à frente por meio da janela móvel de estimação de 36 meses, estão apresentados na Figura 29, juntamente com os valores observados.

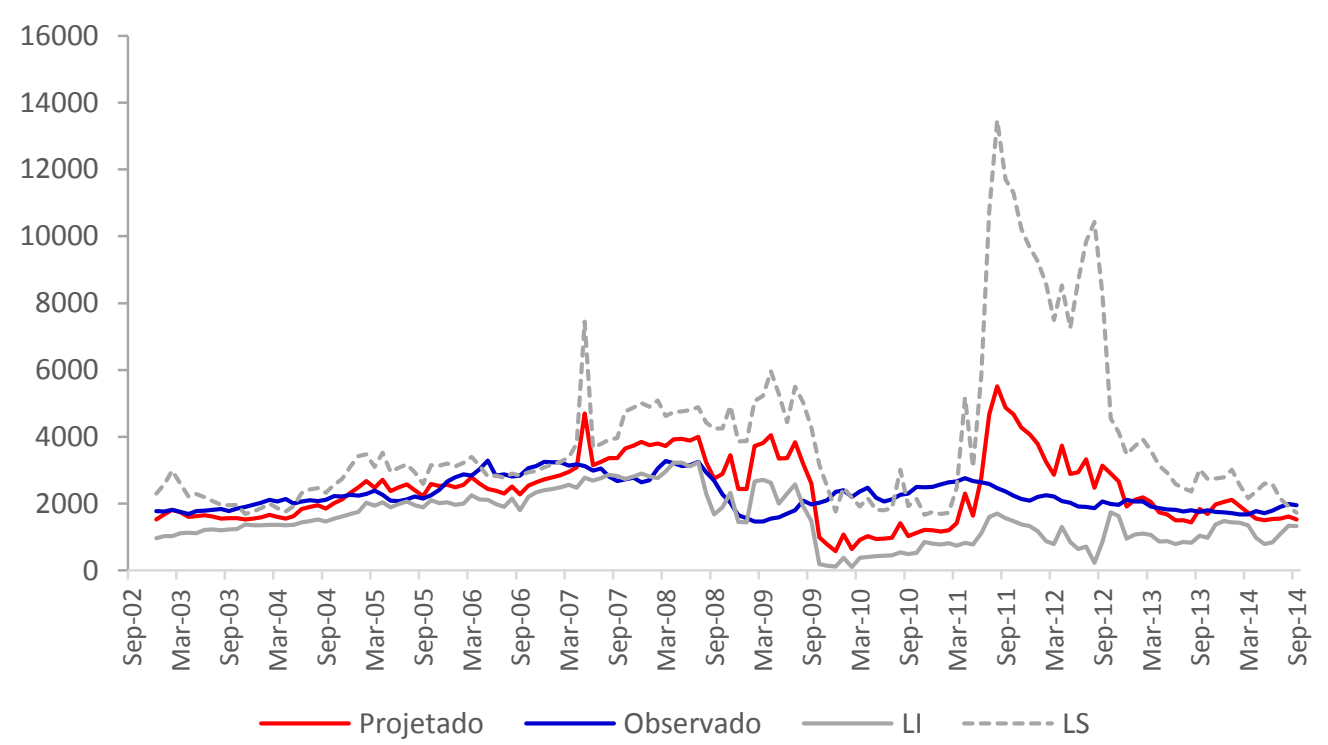

Figura 29 - Modelo Estrutural: Preços Observados e Projetados (Dez/2002 - Set/2014, US\$/t) 


\subsection{Modelos Combinados}

Para cada uma das 60 janelas móveis de estimação, que resultam nos valores projetados 12 meses à frente, de outubro de 2009 a setembro de 2014, foram desenvolvidos 9 modelos combinados. Isso representa 540 estimações, as quais somadas às 710 estimações dos modelos individuais (142 estimações para cada um dos 5 modelos individuais), tem-se um total de 1.250 estimações realizadas.

A descrição dos modelos combinados estão apresentados na Tabela 28.

Tabela 28 - Descrição dos Modelos Combinados

\begin{tabular}{ll}
\hline Modelo & Método para determinação dos pesos das combinações \\
\hline 1. COMB_AVG1 & Média artimética simples dos modelos AR(1), ARIMA, ARMAX1, Estrutural \\
2. COMB_AVG2 & Média artimética simples dos modelos AR(1), ARIMA, Estrutural \\
3. COMB_OLS & Mínimos quadrados ordinários dos modelos AR(1), ARIMA, ARMAX1, Estrutural \\
4. COMB_RMSE & Média ponderada com base no inverso do RMSE do AR(1), ARIMA, Estrutural \\
5. COMB_AICC & Média ponderada com base no AIC dos modelos AR(1), ARIMA, ARMAX1, \\
& Estrutural \\
6. COMB_ERLS & Minimização da soma dos quadrados dos resíduos com a restrição de que a soma dos \\
& pesos seja 1 para AR(1), ARIMA, ARMAX1, Estrutural \\
7. COMB_NERLS & Minimização da soma da soma dos quadrados dos resíduos com a restrição de que a \\
& soma dos pesos seja 1 e não negativa para AR(1), ARIMA, Estrutural \\
8. COMB_NRLS & Minimização da soma da soma dos quadrados dos resíduos com a restrição de que a \\
& soma dos pesos seja não negativa para AR(1), ARIMA, Estrutural \\
9. COMB_ARMAX & Mínimos quadrados ordinários dos modelos ARIMA, ARMAX2 \\
\hline
\end{tabular}

Os resultados dos modelos combinados são apresentados nas seções 5.2.1 a 5.2.9.

\subsubsection{Modelo Combinado 1 (COMB_AVG1)}

O modelo combinado 1 foi realizado por meio da média simples e incluiu $\operatorname{AR}(1), \operatorname{ARMA}(1,10)$, ARMAX1 e Estrutural. Os resultados previstos do modelo combinado 1 para os 60 pontos fora da amostra (outubro de 2009 a setembro de 2014) estão apresentados na Figura 30. 


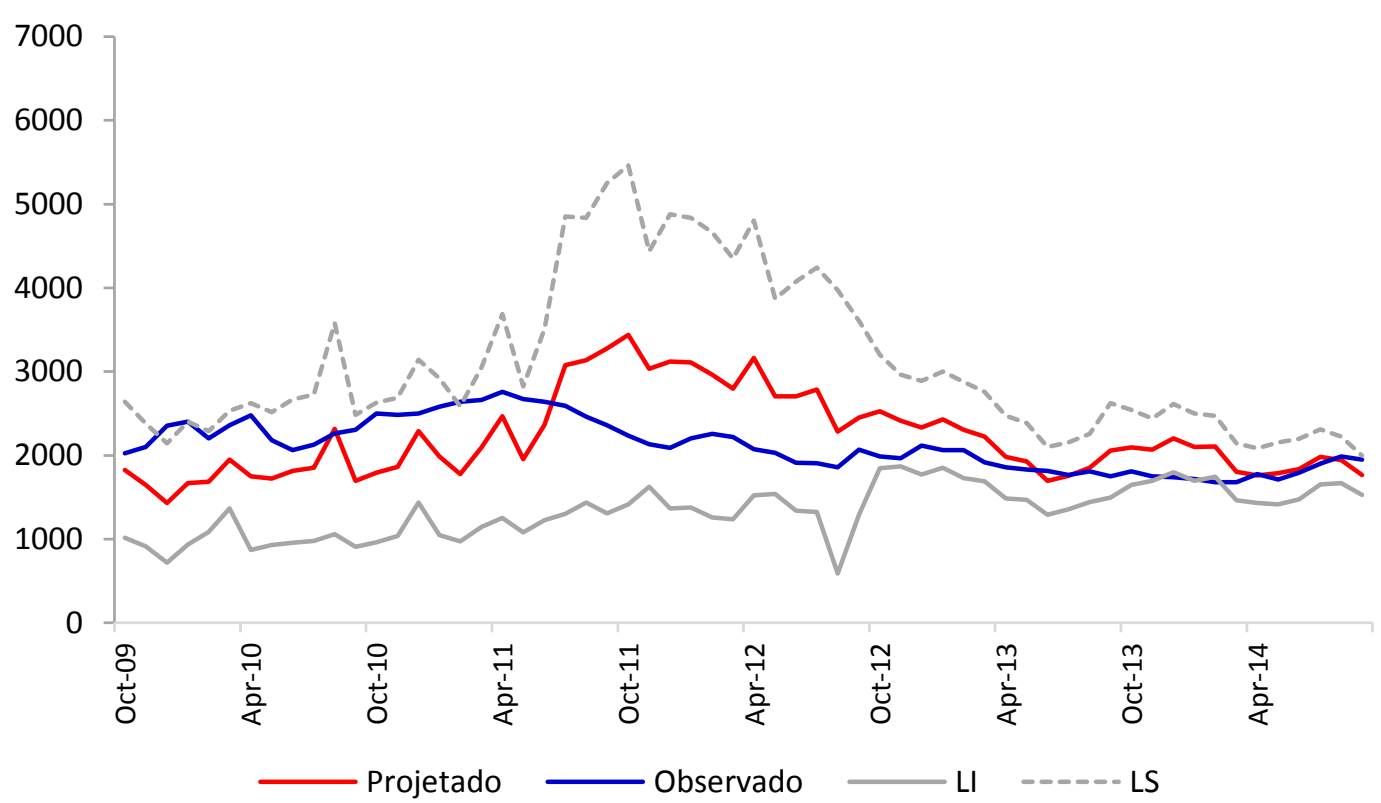

Figura 30 - Resultados do modelo combinado 1 (COMB_AVG1)

Entre outubro de 2009 e maio de 2011 e, entre julho de 2012 até setembro de 2014, o modelo combinado apresentou boa performance de acuracidade, com a amplitude do intervalo de confiança relativo baixa, particularmente em 2013 e de janeiro a setembro de 2014. De junho de 2011 a junho de 2012, a amplitude do intervalo de confiança aumentou significativamente, especialmente em torno de 2011.

A evolução do RMSE ao longo do tempo, ponto a ponto, está mostrado na Figura 31, confirmando boa acuracidade de outubro/2009 a dezembro/2009 e melhoria consistente após outubro/2012. 


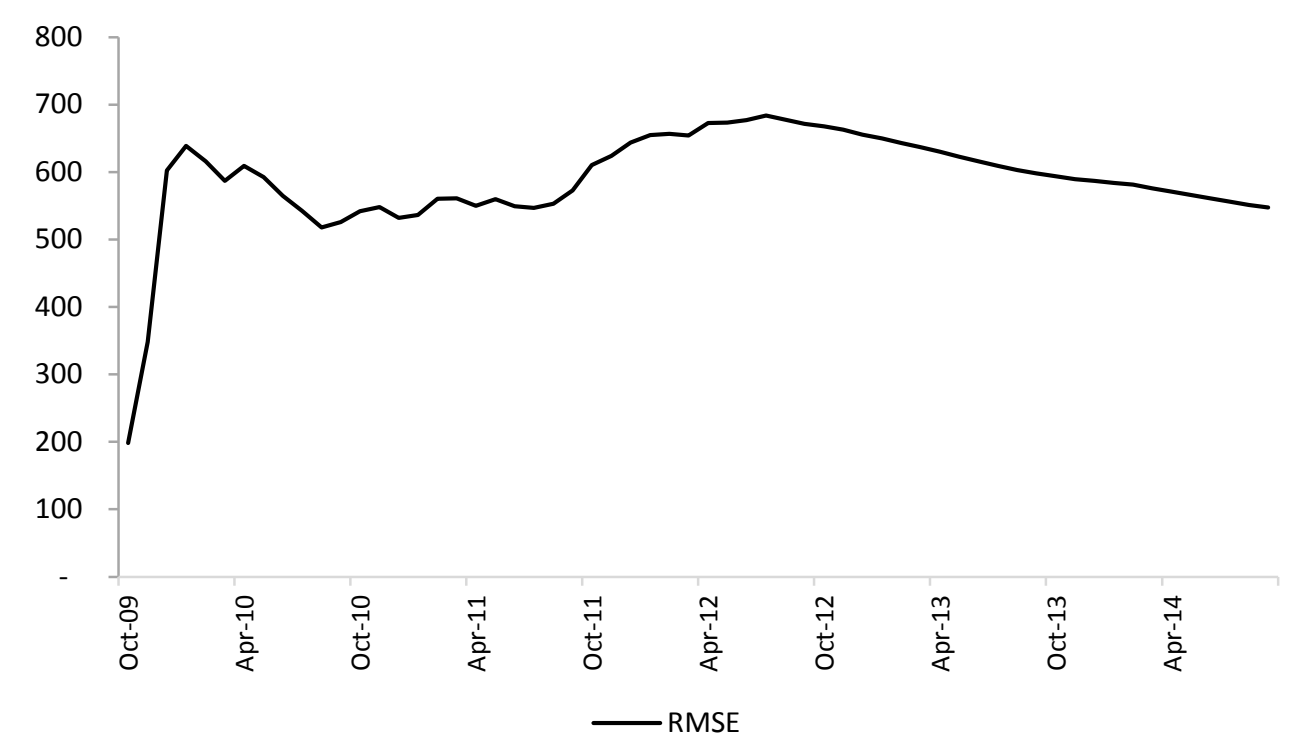

Figura 31 - Evolução do RMSE do modelo combinado 1 (COMB_AVG1)

Os valores das métricas de acuracidade estão indicadas na Tabela 24:

Tabela 29 - Métricas de acuracidade fora da amostra do modelo combinado 1 (COMB_AVG1)

\begin{tabular}{l|r}
\hline Medida & Valor \\
\hline MSE & $299,584.53$ \\
RMSE & 547.34 \\
MAE & 455.91 \\
MAPE & 0.2100 \\
MSPE & 0.0625 \\
RMSPE & 0.2500 \\
Amplitude do IC & $1,753.38$ \\
\hline
\end{tabular}

\subsubsection{Modelo Combinado 2 (COMB_AVG2)}

O modelo combinado 2 foi realizado por meio da média simples e incluiu $\operatorname{AR}(1), \operatorname{ARMA}(1,10)$, e Estrutural. Os resultados previstos do modelo combinado 2 para os 60 pontos fora da amostra (outubro de 2009 a setembro de 2014) estão apresentado na Figura 32. 


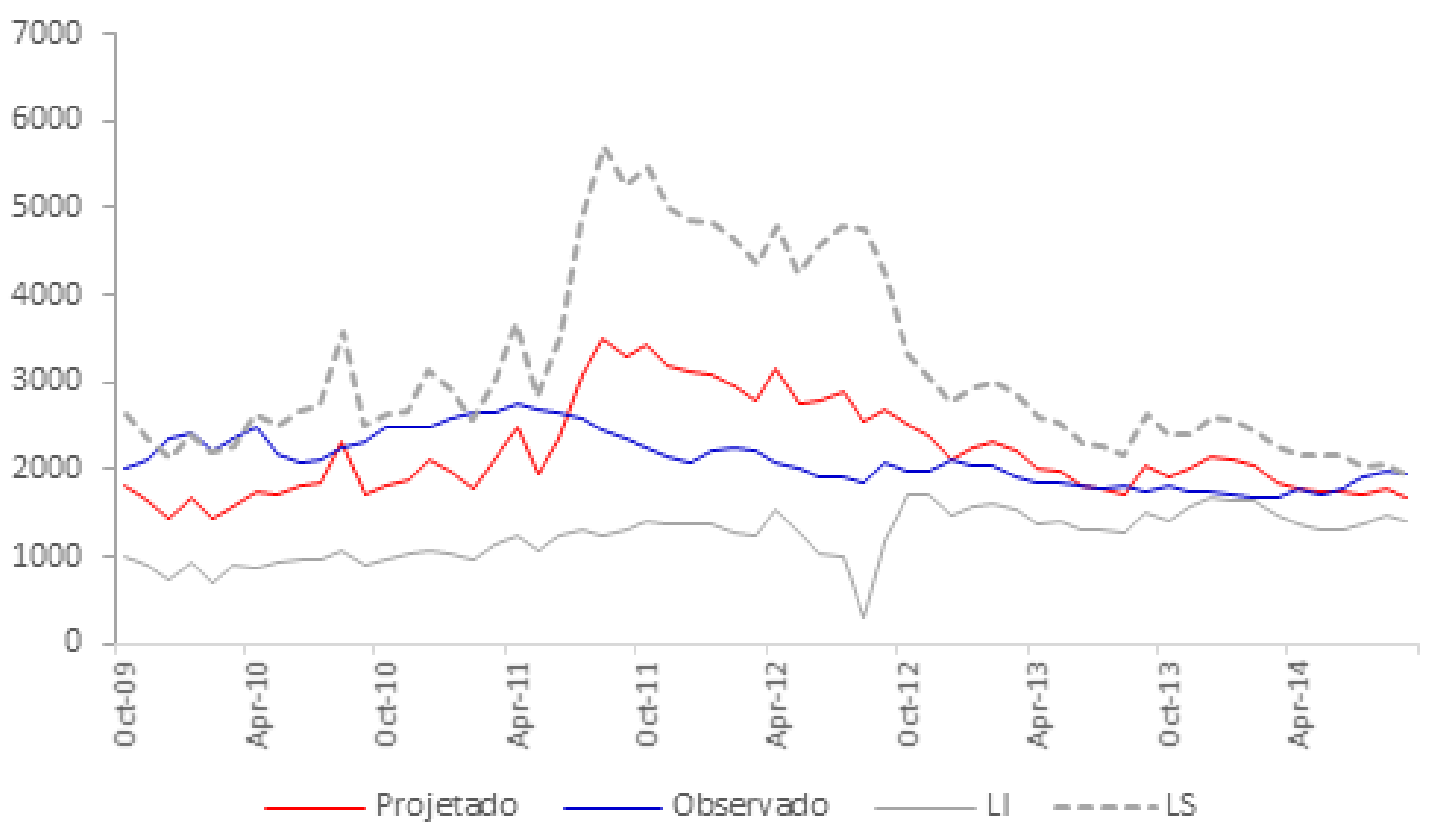

Figura 32 - Resultados do modelo combinado 2 (COMB_AVG2)

Entre outubro de 2009 e maio de 2011 e, entre novembro de 2012 até setembro de 2014, o modelo combinado apresentou boa performance de acuracidade, com a amplitude do intervalo de confiança relativo baixa, particularmente em 2013 e de janeiro a setembro de 2014. De junho de 2011 a junho de 2012, a amplitude do intervalo de confiança aumentou significativamente, especialmente em torno de outubro de 2011. Nota-se que de janeiro a setembro de 2014, a acuracidade foi a melhor ao longo dos cinco anos, com um pequeno intervalo de confiança.

A evolução do RMSE ao longo do tempo, ponto a ponto, está mostrado na Figura 33, confirmando boa acuracidade de outubro/2009 a dezembro/2009 e melhoria consistente após outubro/2012. 


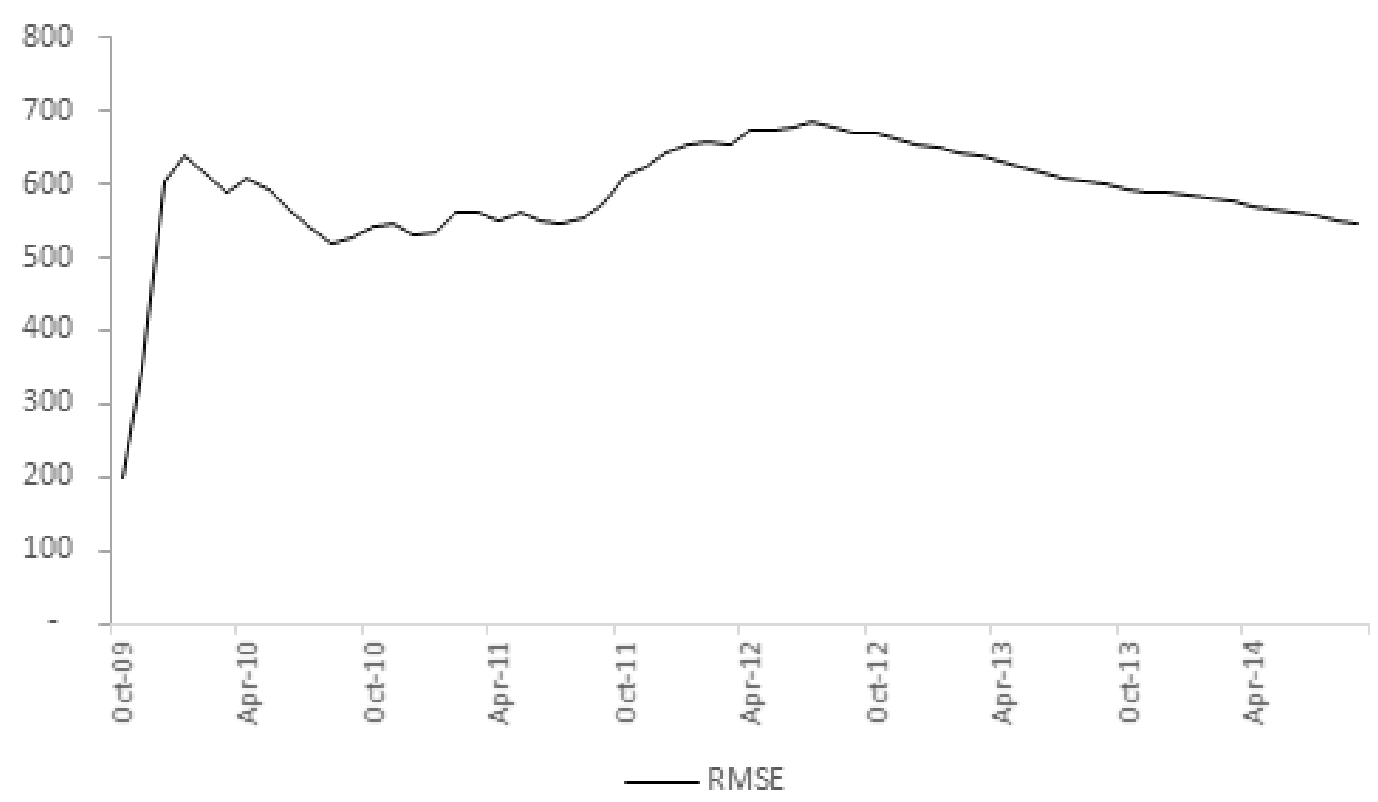

Figura 33 - Evolução do RMSE do modelo combinado 2 (COMB_AVG2)

Os valores das métricas de acuracidade estão indicadas na Tabela 25:

Tabela 30 - Métricas de acuracidade fora da amostra do modelo combinado 2 (COMB_AVG2)

\begin{tabular}{l|r}
\hline Medida & Valor \\
\hline MSE & $299,584.53$ \\
RMSE & 547.34 \\
MAE & 455.91 \\
MAPE & 0.2100 \\
MSPE & 0.0625 \\
RMSPE & 0.2500 \\
Amplitude do IC & $1,753.38$ \\
\hline
\end{tabular}

\subsubsection{Modelo Combinado 3 (COMB_OLS)}

O modelo combinado 3 foi realizado por meio de OLS e incluiu $\operatorname{AR}(1)$, $\operatorname{ARMA}(1,10)$, ARMAX1 e Estrutural. Os resultados previstos do modelo combinado 3 para os 60 pontos fora da amostra (outubro de 2009 a setembro de 2014) estão apresentado na Figura 34. 


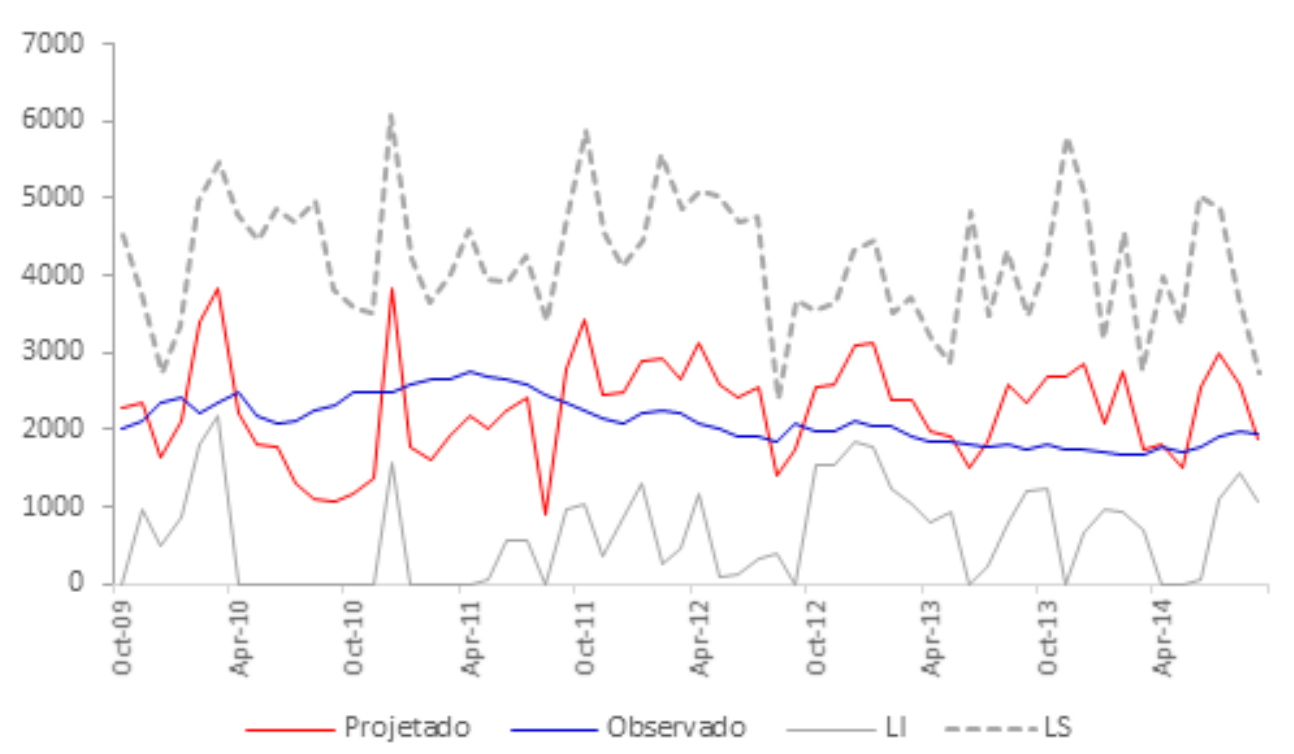

Figura 34 - Resultados do modelo combinado 3 (COMB_OLS)

O intervalo de confiança apresentou variações significativas ao longo do período, principalmente em dezembro/2010, outubro/2011 e novembro/2013. Nos últimos 48 meses. A o valor projetado ficou em geral acima do valor observado.

A evolução do RMSE ao longo do tempo, ponto a ponto, está mostrado na Figura 35, confirmando boa acuracidade de outubro/2009 a dezembro/2009 e melhoria consistente após outubro/2012.

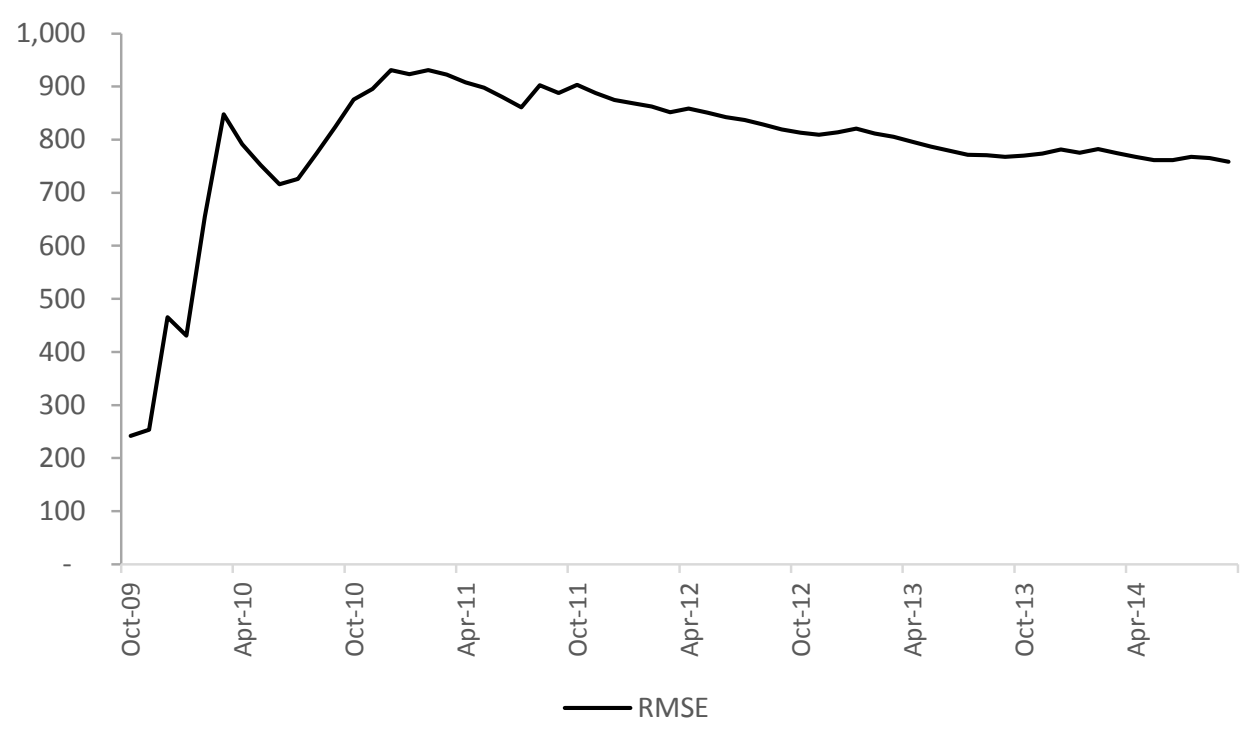

Figura 35 - Evolução do RMSE do modelo combinado 3 (COMB_OLS) 
Os valores das métricas de acuracidade estão indicadas na Tabela 26:

Tabela 31 - Métricas de acuracidade fora da amostra do modelo combinado 3 (COMB_OLS)

\begin{tabular}{l|r}
\hline Medida & Valor \\
\hline MSE & $575,749.72$ \\
RMSE & 758.78 \\
MAE & 648.18 \\
MAPE & 0.3037 \\
MSPE & 0.1246 \\
RMSPE & 0.3531 \\
Amplitude do IC & $3,570.85$ \\
\hline
\end{tabular}

\subsubsection{Modelo Combinado 4 (COMB_RMSE)}

O modelo combinado 4 foi realizado por meio do critério do RMSE e incluiu AR(1), ARMA(1,10), ARMAX1 e Estrutural. Os resultados previstos do modelo combinado para os 60 pontos fora da amostra (outubro de 2009 a setembro de 2014) estão apresentado na Figura 36.

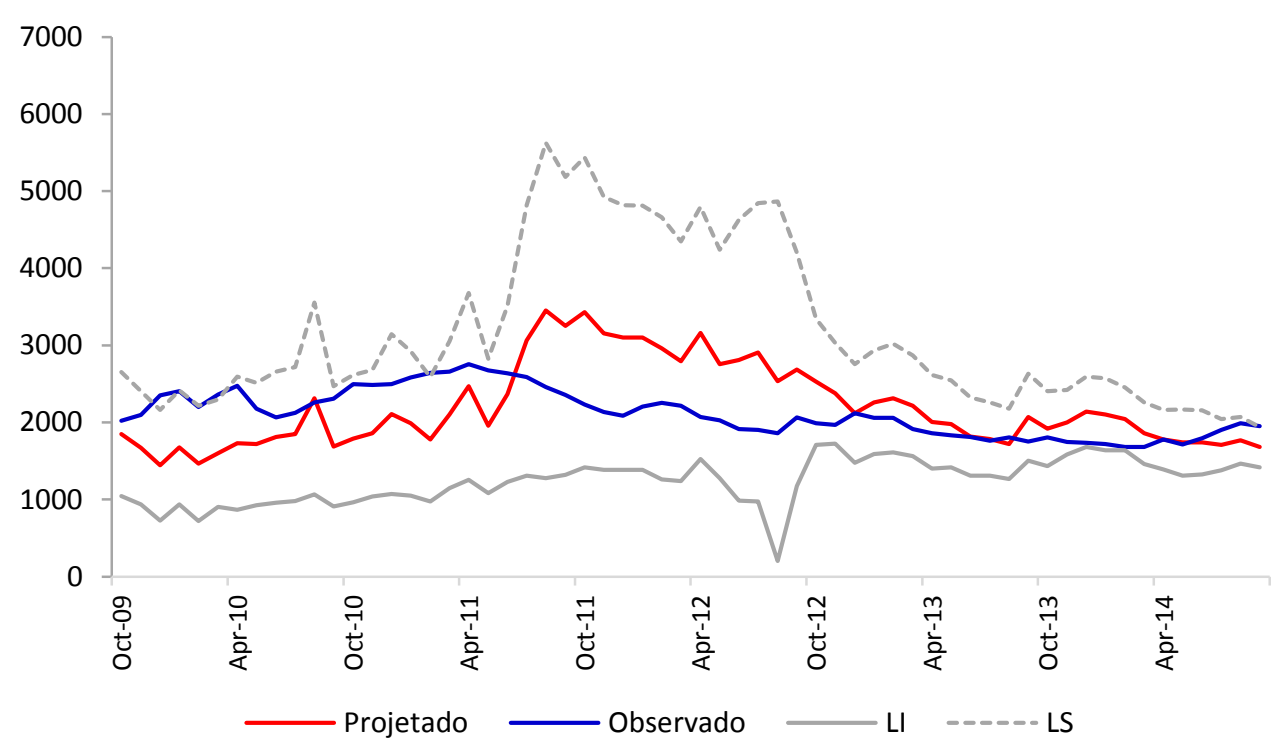

Figura 36 - Resultados do modelo combinado 4 (COMB_RMSE) 
O intervalo de confiança apresentou menor amplitude a partir de dezembro/2012 a setembro/2014. Entre julho de 2011 a outubro de 2012, a amplitude absoluta do intervalo de confiança foi significativa, com um pico em torno de outubro/2011 e agosto/2012.

A evolução do RMSE ao longo do tempo, ponto a ponto, está mostrado na Figura 37, com melhoria consistente após agosto/2012. Menores valores de RMSE são observados no início da amostra (outubro a dezembro/2009), entre agosto/2010 e julho/2011 e após abril/2014.

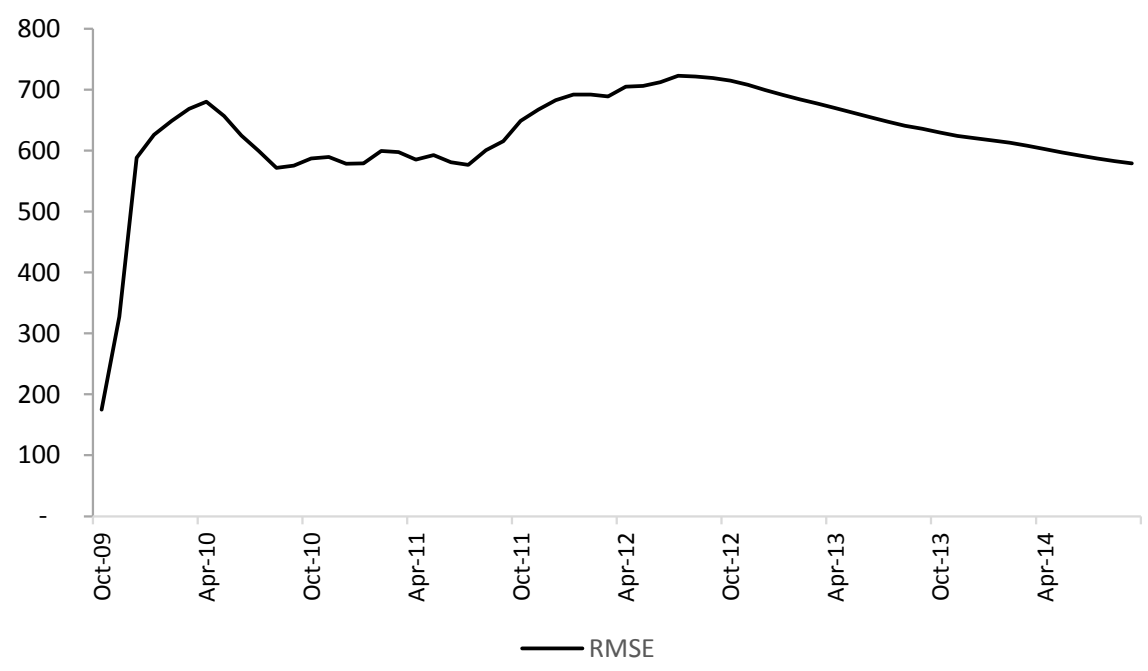

Figura 37 - Evolução do RMSE do modelo combinado 4 (COMB_RMSE)

Os valores das métricas de acuracidade estão indicados na Tabela 27.

Tabela 32 - Métricas de acuracidade fora da amostra do modelo combinado 4 (COMB_RMSE)

\begin{tabular}{l|r}
\hline Medida & Valor \\
\hline MSE & $335,272.77$ \\
RMSE & 579.03 \\
MAE & 480.28 \\
MAPE & 0.2209 \\
MSPE & 0.0699 \\
RMSPE & 0.2645 \\
Amplitude do IC & $1,918.97$ \\
\hline
\end{tabular}




\subsubsection{Modelo Combinado 5 (COMB_AICC)}

O modelo combinado 5 foi realizado por meio do critério de informação de Akaike e incluiu AR(1), ARMA(1,10), ARMAX1 e Estrutural. Os resultados previstos do modelo combinado para os 60 pontos fora da amostra (outubro de 2009 a setembro de 2014) estão apresentados na Figura 38.

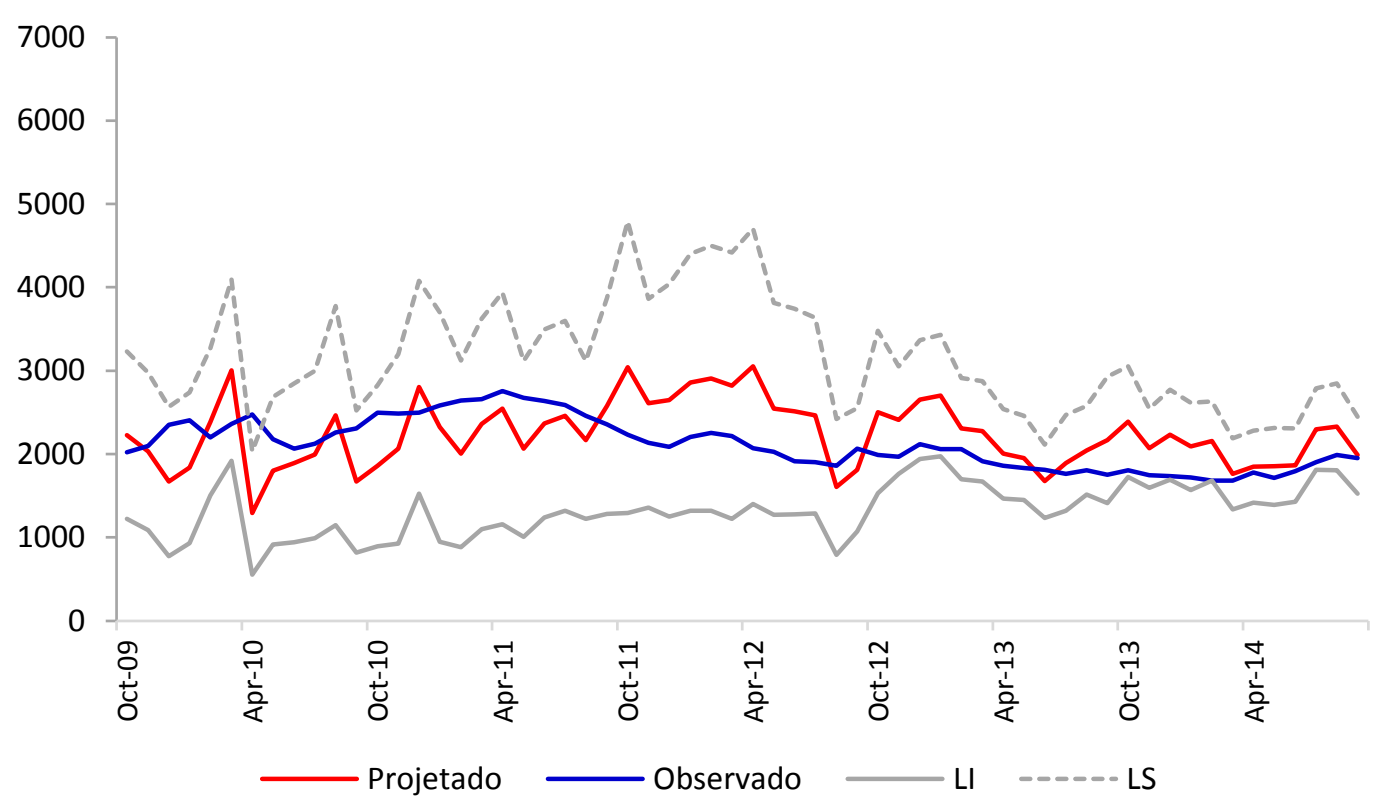

Figura 38 - Resultados do modelo combinado 5 (COMB_AICC)

O modelo apresentou boa acuracidade em 2013, com erro e intervalo de confiança menor ainda de fevereiro a setembro/2014. O intervalo de confiança apresentou maior amplitude a partir de ourubro/2011 a março/2012. O RMSE e MSE médio foram em torno de \$400/t, que é um valor adequado, considerando que são previsões 12 meses à frente.

A evolução do RMSE ao longo do tempo, ponto a ponto, está mostrado na Figura 39, com melhoria consistente após agosto/2012. Menores valores de RMSE são observados no início da amostra (outubro/2009 a março/2010) e de janeiro a setembro/2014. 


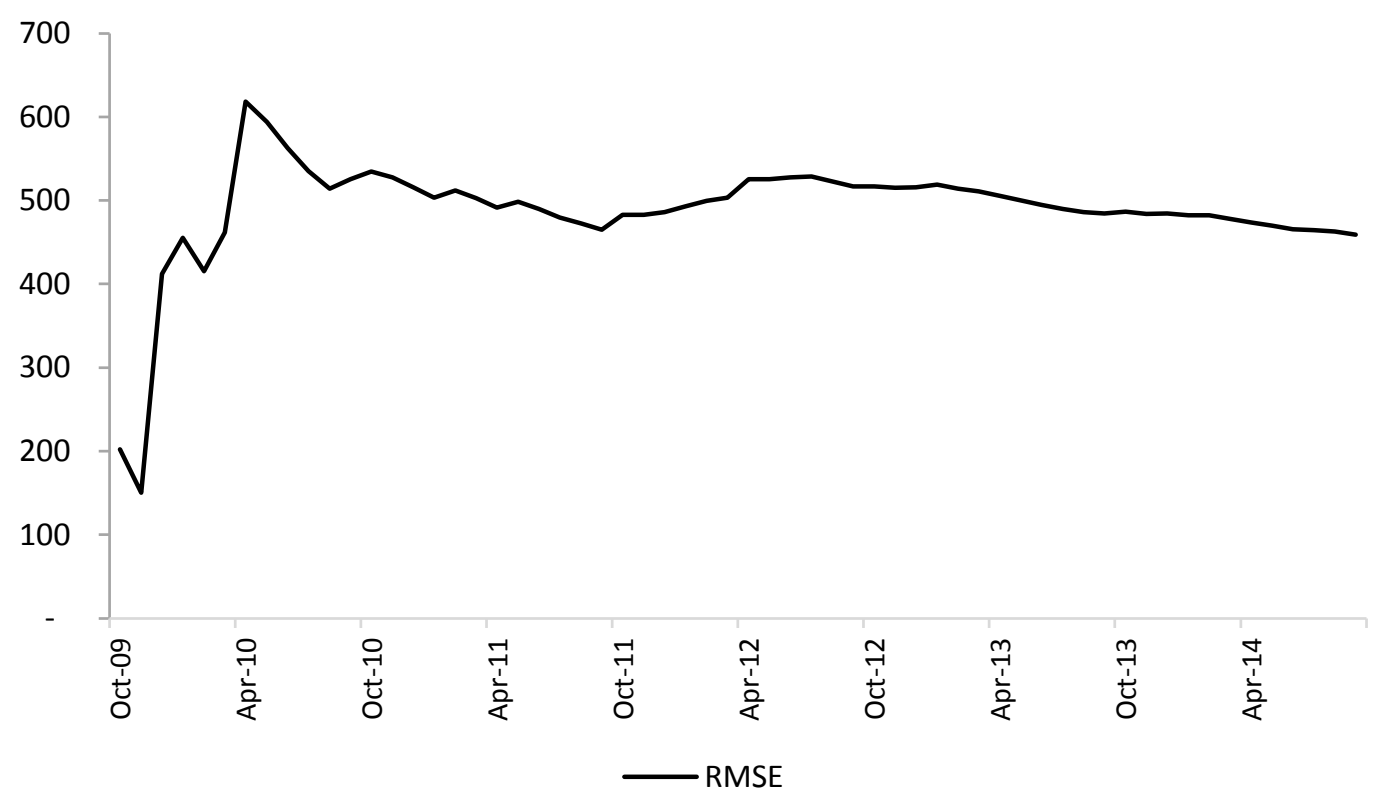

Figura 39 - Evolução do RMSE do modelo combinado 5 (COMB_AICC)

Os valores das métricas de acuracidade estão indicadas na Tabela 28.

Tabela 33 - Métricas de acuracidade fora da amostra do modelo combinado 5 (COMB_AICC)

\begin{tabular}{l|r}
\hline Medida & Valor \\
\hline MSE & $210,630.33$ \\
RMSE & 458.94 \\
MAE & 392.73 \\
MAPE & 0.1844 \\
MSPE & 0.0454 \\
RMSPE & 0.2132 \\
Amplitude do IC & $1,836.03$ \\
\hline
\end{tabular}

\subsubsection{Modelo Combinado 6 (COMB_ERLS)}

O modelo combinado 6 foi realizado por meio de mínimos quadrados igualmente restritos e incluiu AR(1), ARMA(1,10), ARMAX1 e Estrutural. Os resultados previstos do modelo combinado para os 60 pontos fora da amostra (outubro de 2009 a setembro de 2014) estão apresentado na Figura 40. 


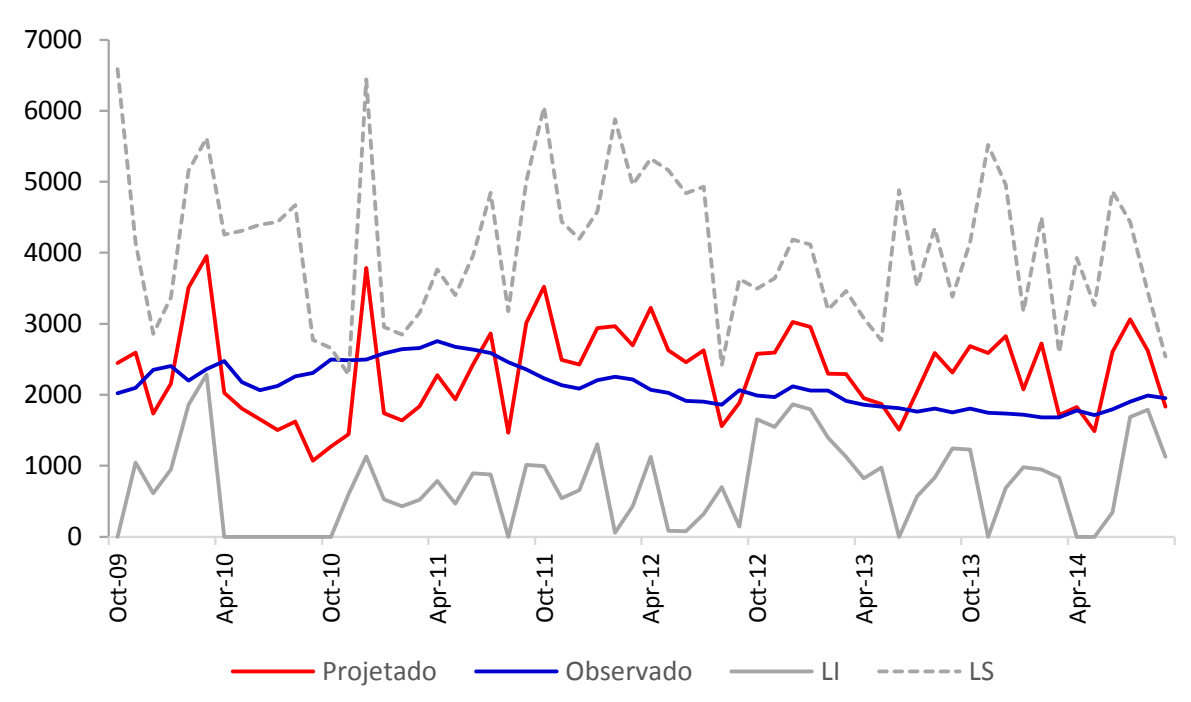

Figura 40 - Resultados do modelo combinado 6 (COMB_ERLS)

A partir de setembro/11, os valores previstos ficaram com maior frequência acima dos valores observados. O limite inferior do intervalo de confiança foi zero em vários meses e o intervalo superior apresentou vários picos ao longo da amostra, como em dezembro/2009, dezembro/2010 e outubro/2011. O RMSE e MSE médio foram em torno de \$700/t, mostrando, na media, uma maior variabilidade das projeções.

A evolução do RMSE ao longo do tempo, ponto a ponto, está mostrado na Figura 41.

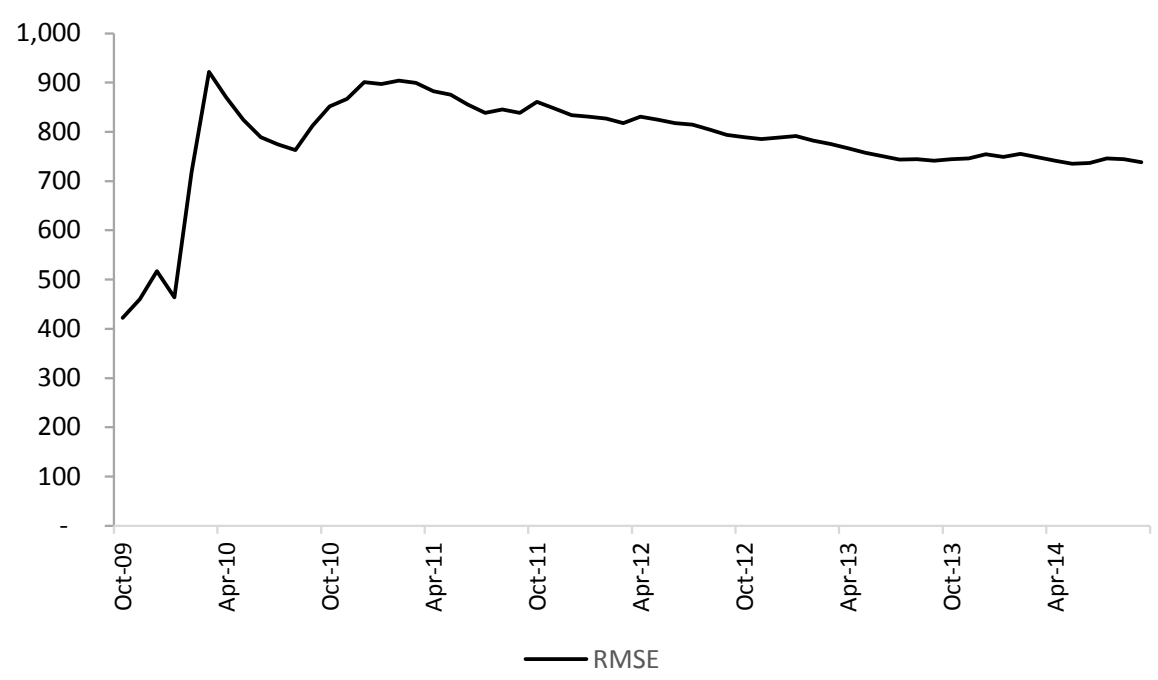

Figura 41 - Evolução do RMSE do modelo combinado 6 (COMB_ERLS) 
Os valores das métricas de acuracidade estão indicadas na Tabela 29.

Tabela 34 - Métricas de acuracidade fora da amostra do modelo combinado 6 (COMB_ERLS)

\begin{tabular}{l|r}
\hline Medida & Valor \\
\hline MSE & $545,235.78$ \\
RMSE & 738.40 \\
MAE & 638.69 \\
MAPE & 0.3003 \\
MSPE & 0.1196 \\
RMSPE & 0.3459 \\
Amplitude do IC & $3,350.75$ \\
\hline
\end{tabular}

\subsubsection{Modelo Combinado 7 (COMB_NERLS)}

O modelo combinado 7 foi realizado por meio de mínimos quadrados igualmente restritos e não negativos e incluiu $\operatorname{AR}(1), \operatorname{ARMA}(1,10)$ e Estrutural. Os resultados previstos do modelo combinado para os 60 pontos fora da amostra (outubro de 2009 a setembro de 2014) estão apresentado na Figura 42.

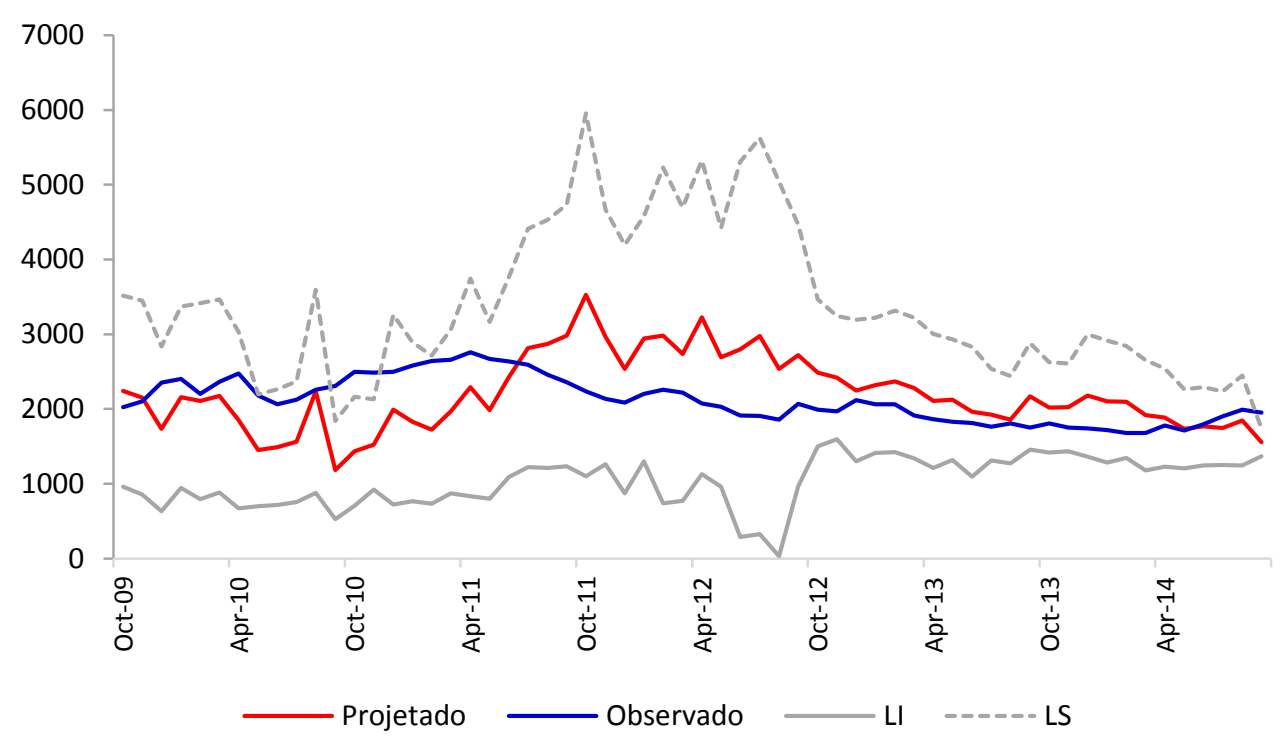

Figura 42 - Resultados do modelo combinado 7 (COMB_NERLS) 
O modelo apresentou boa acuracidade a partir de novembro/2012 até o final da amostra, em setembro/2014, bem como no início da amostra, de outubro/2009 a setembro/2010. O intervalo de confiança apresentou maior amplitudes entre setembro/2011 e setembro/2012. O RMSE e MSE médio foram, respectivamante, 569 e 473. A amplitude média ao longo de toda a amostra foi de 2.332 .

A evolução do RMSE ao longo do tempo, ponto a ponto, está mostrado na Figura 43.

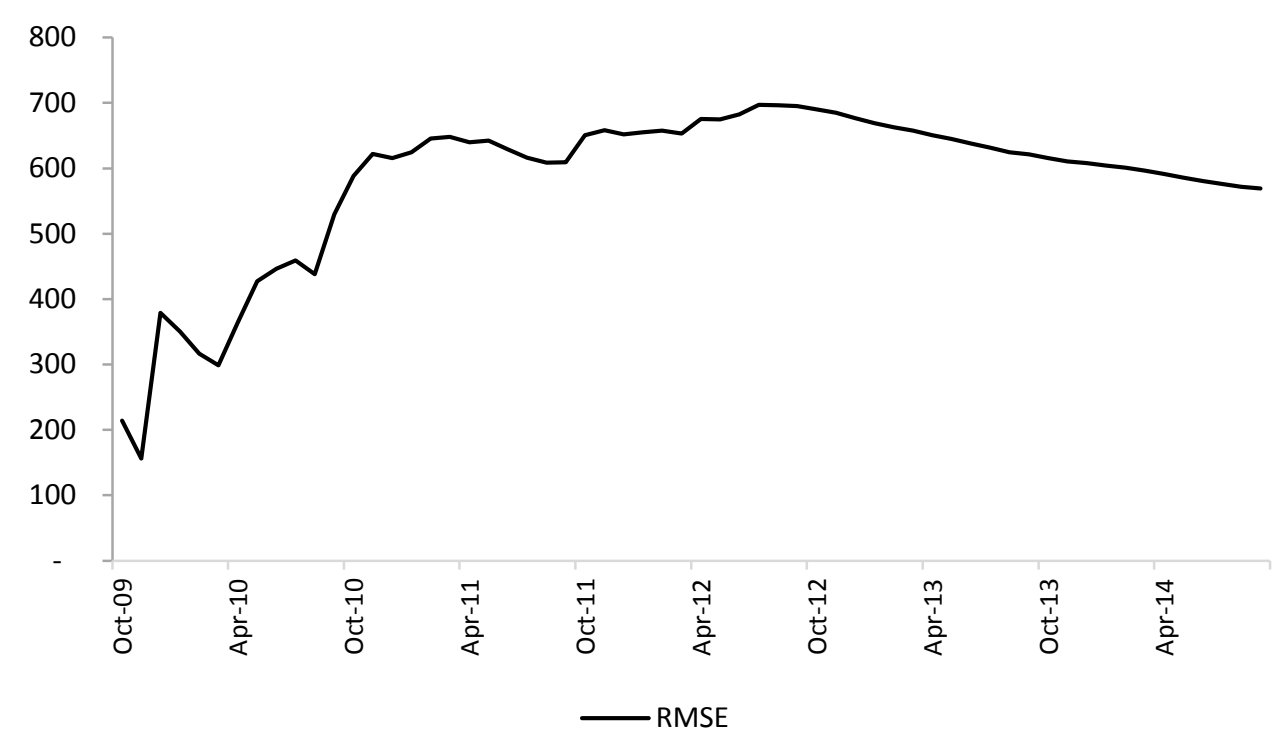

Figura 43 - Evolução do RMSE do modelo combinado 7 (COMB_NERLS)

Os valores das métricas de acuracidade estão indicadas na Tabela 30:

Tabela 35 - Métricas de acuracidade fora da amostra do modelo combinado 7 (COMB_NERLS)

\begin{tabular}{l|r}
\hline Medida & Valor \\
\hline MSE & $323,744.74$ \\
RMSE & 568.99 \\
MAE & 472.77 \\
MAPE & 0.2195 \\
MSPE & 0.0682 \\
RMSPE & 0.2611 \\
Amplitude do IC & $2,332.36$ \\
\hline
\end{tabular}




\subsubsection{Modelo Combinado 8 (COMB_NRLS)}

O modelo combinado 8 foi realizado por meio de mínimos quadrados não negativos e incluiu $\operatorname{AR}(1), \operatorname{ARMA}(1,10)$ e Estrutural. Os resultados previstos do modelo combinado para os 60 pontos fora da amostra (outubro de 2009 a setembro de 2014) estão apresentado na Figura 44.

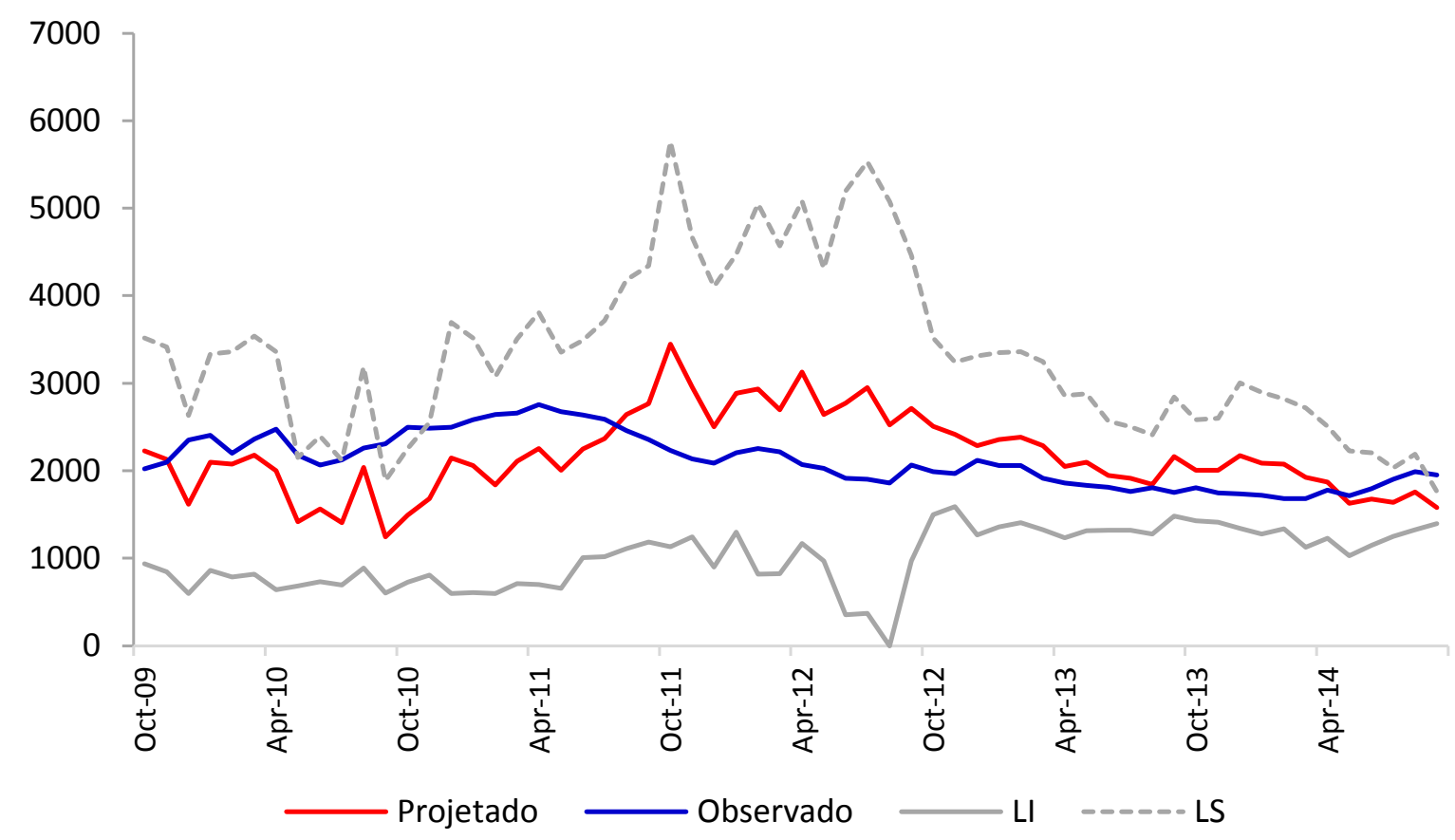

Figura 44 - Resultados do modelo combinado 8 (COMB_NRLS)

O modelo apresentou boa acuracidade a partir de janeiro/2013 até o final da amostra, em setembro/2014, bem como no início da amostra, de outubro/2009 a março/2010. O intervalo de confiança apresentou maior amplitude entre outubro/2011 e agosto/2012. O RMSE e MSE médio foram, respectivamente, 537 e 455 . A amplitude média ao longo de toda a amostra foi de 2.330. A evolução do RMSE ao longo do tempo, ponto a ponto, está mostrado na Figura 45. 


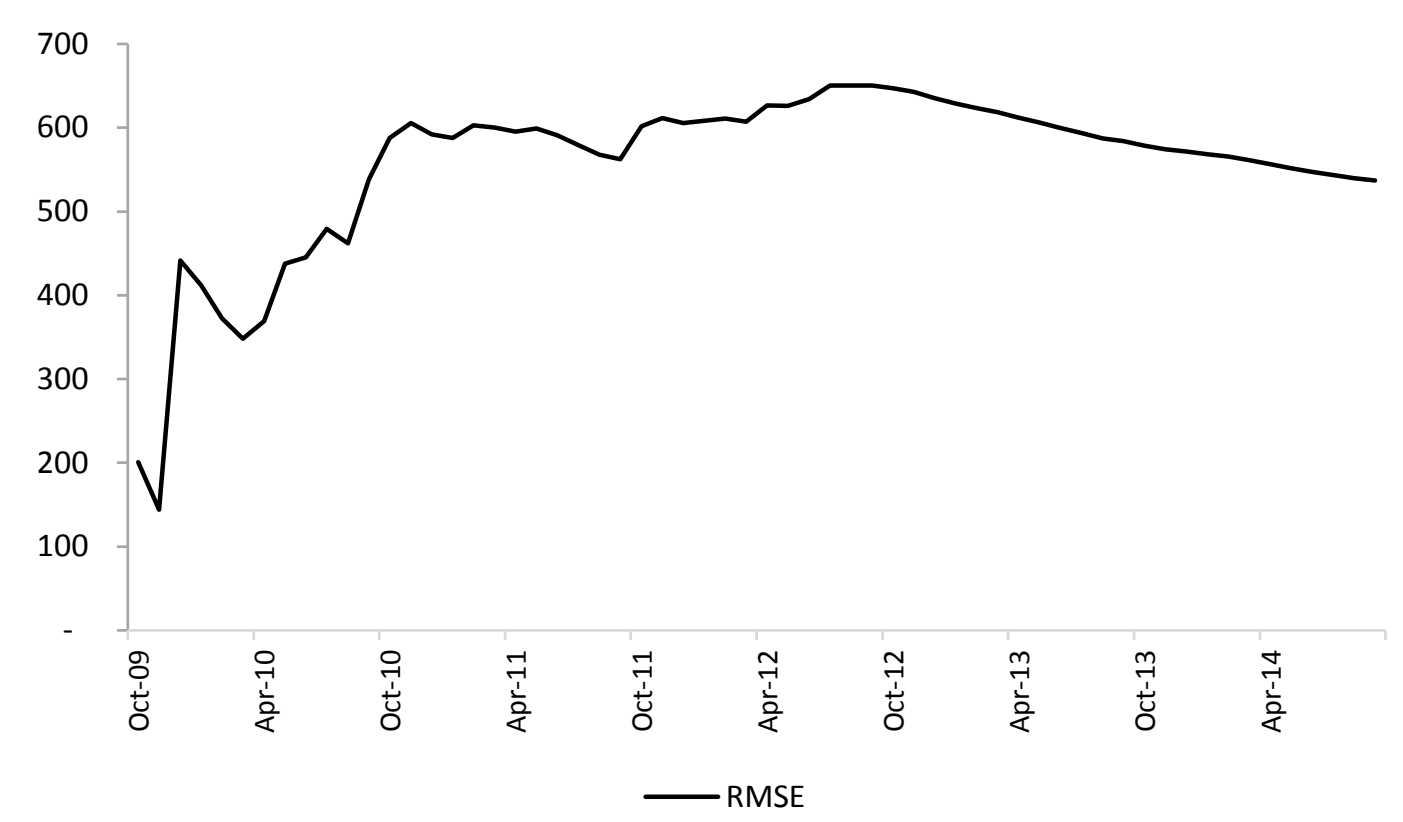

Figura 45 - Evolução do RMSE do modelo combinado 8 (COMB_NRLS)

Os valores das métricas de acuracidade estão indicadas na Tabela 31:

Tabela 36 - Métricas de acuracidade fora da amostra do modelo combinado 8 (COMB_NRLS)

\begin{tabular}{l|r}
\hline Medida & Valor \\
\hline MSE & $288,587.58$ \\
RMSE & 537.20 \\
MAE & 454.56 \\
MAPE & 0.2122 \\
MSPE & 0.0618 \\
RMSPE & 0.2487 \\
Amplitude do IC & $2,330.19$ \\
\hline
\end{tabular}

\subsubsection{Modelo Combinado 9 (COMB_ARMAX)}

O modelo combinado 9 foi realizado por meio de OLS e incluiu ARMA(1,10) e ARMAX2. Os resultados previstos do modelo combinado para os 60 pontos fora da amostra (outubro de 2009 a setembro de 2014) estão apresentado na Figura 46. 


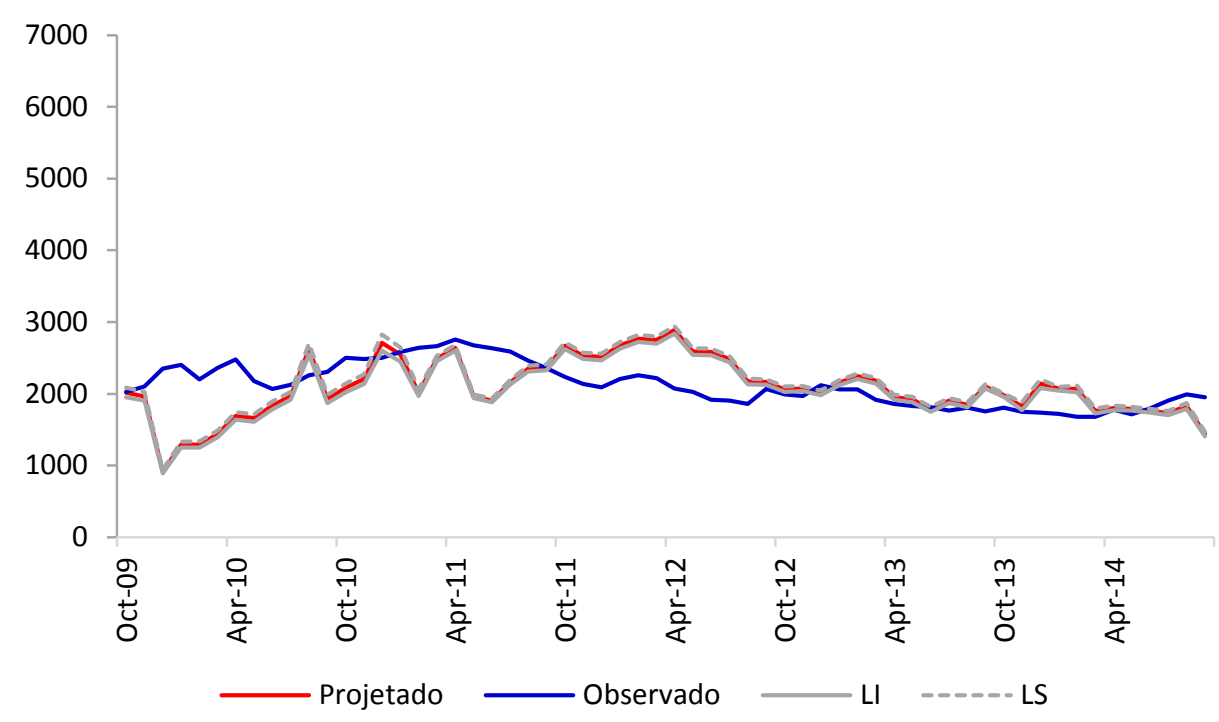

Figura 46 - Resultados do modelo combinado 9 (COMB_ARMAX)

O modelo apresentou melhor acuracidade a partir de setembro/2012 até o final da amostra, em setembro/2014, e de maio/2010 a setembro/2010, a acuracidade do modelo também foi boa. Maiores desvios ocorrem em poucos meses, de outubro/2009 a abril/2010. O intervalo de confiança ao longo da amostra foi pequeno, com uma média de 89. O RMSE e MSE médio foram, respectivamente, 456 e 343.

A evolução do RMSE ao longo do tempo, ponto a ponto, está mostrado na Figura 47, onde se nota uma redução contínua do RMSE a partir de abril/2010.

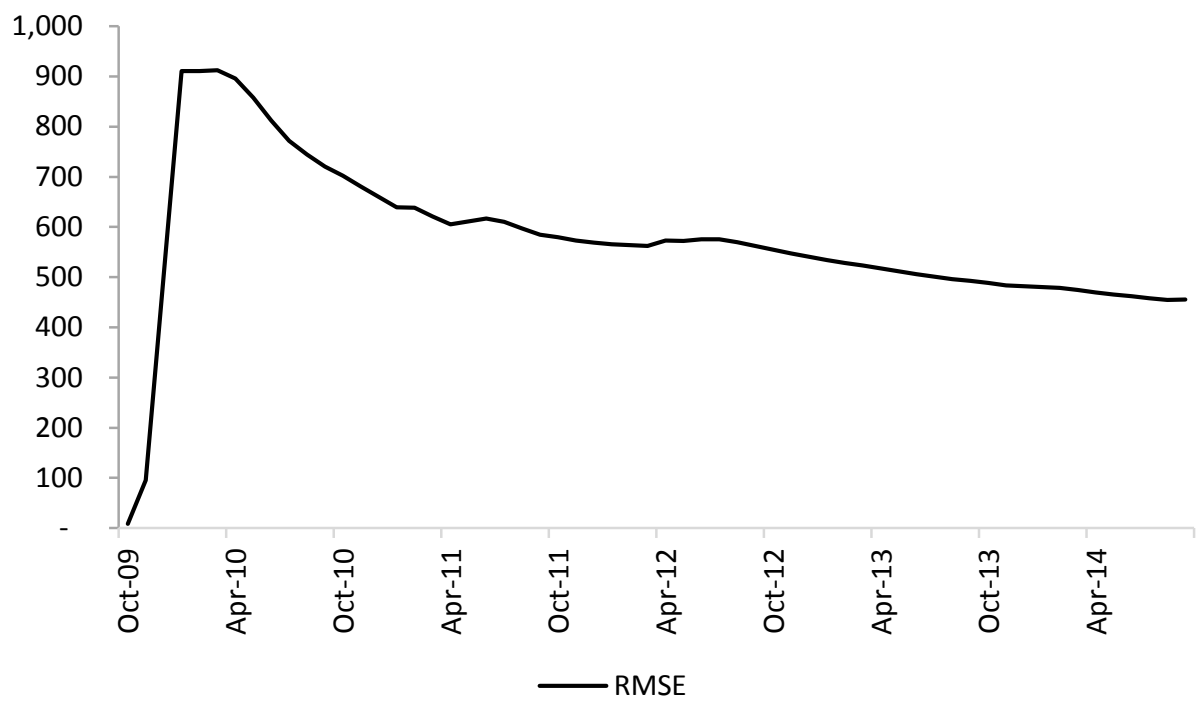

Figura 47 - Evolução do RMSE do modelo combinado 9 (COMB_ARMAX) 
Os valores das métricas de acuracidade estão indicadas Tabela 32:

Tabela 37 - Métricas de acuracidade fora da amostra do modelo combinado 9 (COMB_ARMAX)

\begin{tabular}{l|r}
\hline Medida & Valor \\
\hline MSE & $207,764.76$ \\
RMSE & 455.81 \\
MAE & 343.49 \\
MAPE & 0.1580 \\
MSPE & 0.0418 \\
RMSPE & 0.2045 \\
Amplitude do IC & 89.22 \\
\hline
\end{tabular}

\subsection{Comparação dos Modelos}

\subsubsection{Métricas Tradicionais}

As tabelas 33, 34 e 35 comparam o desempenho preditivo dos modelos através das métricas tradicionais.

Tabela 38 - Comparação de Modelos - 36 meses mais recentes

\begin{tabular}{lcccccrr}
\hline Modelo & MSE & RMSE & MAE & MAPE & \multicolumn{1}{l}{ MSPE } & \multicolumn{1}{l}{ RMSPE } & \multicolumn{1}{l}{ Amp. IC } \\
\hline COMB. 9 & $\mathbf{7 1 . 1 3 5 , 5 4}$ & $\mathbf{2 6 6 , 7 1}$ & $\mathbf{1 6 3 , 5 9}$ & $\mathbf{0 , 0 8 3 2}$ & $\mathbf{0 , 0 1 8 0}$ & $\mathbf{0 , 1 3 4 2}$ & $\mathbf{4 6 , 9 8}$ \\
ARIM AX2 & $71.277,47$ & 266,98 & 163,81 & 0,0833 & 0,0180 & 0,1343 & 46,41 \\
ARM A & $89.531,99$ & 299,22 & 204,89 & 0,1055 & 0,0237 & 0,1538 & $1.415,12$ \\
COMB. 5 & $124.176,27$ & 352,39 & 237,20 & 0,1204 & 0,0310 & 0,1760 & 984,25 \\
AR(1) & $134.467,77$ & 366,70 & 233,77 & 0,1190 & 0,0338 & 0,1839 & $1.600,15$ \\
ARIM AX1 & $150.370,41$ & 387,78 & 263,33 & 0,1356 & 0,0398 & 0,1996 & 858,03 \\
COMB. 8 & $162.205,55$ & 402,75 & 258,90 & 0,1311 & 0,0400 & 0,2000 & $1.370,05$ \\
COMB. 1 & $168.288,02$ & 410,23 & 247,04 & 0,1237 & 0,0403 & 0,2007 & 969,58 \\
COMB. 7 & $175.182,55$ & 418,55 & 263,06 & 0,1329 & 0,0430 & 0,2073 & $1.407,42$ \\
COMB. 4 & $183.683,90$ & 428,58 & 255,25 & 0,1278 & 0,0442 & 0,2102 & $1.108,55$ \\
COMB. 2 & $185.268,43$ & 430,43 & 255,61 & 0,1279 & 0,0445 & 0,2109 & $1.107,92$ \\
COMB. 3 & $260.321,80$ & 510,22 & 338,44 & 0,1751 & 0,0702 & 0,2650 & $2.033,84$ \\
COMB. 6 & $263.861,42$ & 513,67 & 339,07 & 0,1753 & 0,0708 & 0,2661 & $1.966,39$ \\
Estrutural & $487.983,25$ & 698,56 & 384,84 & 0,1903 & 0,1119 & 0,3345 & $2.332,31$ \\
\hline
\end{tabular}


Tabela 39 - Comparação de Modelos - 48 meses mais recentes

\begin{tabular}{lccccccr}
\hline Modelo & \multicolumn{1}{c}{ MSE } & \multicolumn{1}{c}{ RMSE } & MAE & MAPE & MSPE & RMSPE & \multicolumn{1}{c}{ Amp. IC } \\
\hline ARIM AX2 & $103.882,99$ & 322,31 & 227,77 & 0,1079 & 0,0228 & 0,1510 & 66,98 \\
COMB. 9 & $\mathbf{1 0 3 . 9 0 9 , 7 2}$ & $\mathbf{3 2 2 , 3 5}$ & $\mathbf{2 2 7 , 6 9}$ & $\mathbf{0 , 1 0 7 9}$ & $\mathbf{0 , 0 2 2 8}$ & $\mathbf{0 , 1 5 0 9}$ & $\mathbf{6 8 , 3 4}$ \\
ARMA & $133.436,80$ & 365,29 & 290,72 & 0,1385 & 0,0301 & 0,1735 & $1.996,53$ \\
AR(1) & $151.691,27$ & 389,48 & 283,13 & 0,1382 & 0,0364 & 0,1907 & $2.441,62$ \\
COMB. 5 & $155.459,46$ & 394,28 & 308,68 & 0,1482 & 0,0357 & 0,1889 & $1.453,90$ \\
ARIMAX1 & $186.738,60$ & 432,13 & 334,44 & 0,1631 & 0,0452 & 0,2126 & 966,57 \\
COMB. 8 & $230.697,47$ & 480,31 & 365,82 & 0,1727 & 0,0504 & 0,2245 & $1.899,86$ \\
COMB. 1 & $244.254,78$ & 494,22 & 362,38 & 0,1689 & 0,0521 & 0,2283 & $1.432,48$ \\
COMB. 7 & $267.624,95$ & 517,32 & 388,50 & 0,1820 & 0,0572 & 0,2391 & $1.898,55$ \\
COMB. 4 & $269.084,75$ & 518,73 & 378,06 & 0,1760 & 0,0576 & 0,2399 & $1.590,90$ \\
COMB. 2 & $273.061,31$ & 522,55 & 379,88 & 0,1767 & 0,0583 & 0,2414 & $1.594,63$ \\
COMB. 6 & $413.132,64$ & 642,75 & 498,68 & 0,2380 & 0,0940 & 0,3066 & $2.587,16$ \\
COMB. 3 & $439.887,15$ & 663,24 & 509,21 & 0,2421 & 0,0983 & 0,3136 & $2.802,29$ \\
Estrutural & $1.021 .803,96$ & $1.010,84$ & 673,29 & 0,3045 & 0,1977 & 0,4446 & $3.155,47$ \\
\hline
\end{tabular}

Tabela 40 - Comparação de Modelos - 60 meses mais recentes

\begin{tabular}{llcccccr}
\hline Modelo & MSE & RMSE & MAE & MAPE & MSPE & RMSPE & Amp. IC \\
\hline ARM A & $143.099,83$ & 378,29 & 328,17 & 0,1556 & 0,0322 & 0,1793 & $2.614,87$ \\
ARIMAX2 & $206.498,50$ & 454,42 & 342,84 & 0,1577 & 0,0416 & 0,2039 & 87,60 \\
COMB. 9 & $\mathbf{2 0 7 . 7 6 4 , 7 6}$ & $\mathbf{4 5 5 , 8 1}$ & $\mathbf{3 4 3 , 4 9}$ & $\mathbf{0 , 1 5 8 0}$ & $\mathbf{0 , 0 4 1 8}$ & $\mathbf{0 , 2 0 4 5}$ & $\mathbf{8 9 , 2 2}$ \\
COMB. 5 & $210.630,33$ & 458,94 & 392,73 & 0,1844 & 0,0454 & 0,2132 & $1.836,03$ \\
AR(1) & $213.865,76$ & 462,46 & 375,73 & 0,1784 & 0,0479 & 0,2189 & $3.057,61$ \\
ARIMAX1 & $272.712,62$ & 522,22 & 436,45 & 0,2071 & 0,0607 & 0,2463 & $1.063,25$ \\
COMB. 8 & $288.587,58$ & 537,20 & 454,56 & 0,2122 & 0,0618 & 0,2487 & $2.330,19$ \\
COMB. 1 & $299.584,53$ & 547,34 & 455,91 & 0,2100 & 0,0625 & 0,2500 & $1.753,38$ \\
COMB. 7 & $323.744,74$ & 568,99 & 472,77 & 0,2195 & 0,0682 & 0,2611 & $2.332,36$ \\
COMB. 4 & $335.272,77$ & 579,03 & 480,28 & 0,2209 & 0,0699 & 0,2645 & $1.918,97$ \\
COMB. 2 & $340.748,18$ & 583,74 & 483,51 & 0,2222 & 0,0710 & 0,2664 & $1.923,52$ \\
COMB. 6 & $545.235,78$ & 738,40 & 638,69 & 0,3003 & 0,1196 & 0,3459 & $3.350,75$ \\
COMB. 3 & $575.749,72$ & 758,78 & 648,18 & 0,3037 & 0,1246 & 0,3531 & $3.570,85$ \\
Estrutural & $1.366 .861,49$ & $1.169,13$ & 931,70 & 0,4198 & 0,2658 & 0,5155 & $3.530,25$ \\
\hline
\end{tabular}

Para os 36 meses mais recentes (outubro/2011 a setembro/2014), a combinação 9 apresentou o melhor desempenho preditivo em todas as métricas, seguido do ARMAX2 e ARMA. A combinação 9 é obtida por OLS dos modelos ARMA e ARMAX2. Para os 48 meses mais recentes (outubro/2010 a setembro/2014), o melhor modelo foi o ARMAX2, seguido da combinação 9 e do ARMA. Para os 60 meses mais recentes (outubro/2009 a setembro/2014), ocorre uma nova inversão, e o ARMA apresenta melhor desempenho, seguido do ARMAX2 e da combinação 9.

Por outro lado, a amplitude do intervalo de confiança do ARMA é muito elevada (cerca de $1.400,2.000$ e 2.600 para 36, 48 e 60 meses, respectivamente) em relação à combinação 9 e ao ARMAX2. As amplitudes do modelo combinado 9 e do ARMAX2 são semelhantes e as mais 
baixas de todos os modelos, sendo em torno de 46, 76, 88 (US\$/t), respectivamente, para 36, 48 e 60 meses.

Os dois outros melhores modelos incluem a combinação 5 e o AR(1). A combinação 5 é obtida por meio do método do critério de informação de Akaike, incluindo os modelos AR(1), ARMA, ARMAX1 e Estrutural. A combinação 5 é o quarto melhor modelo para 36 e 60 meses mais recentes, seguido do AR(1). Para 48 meses, ocorre o inverso, sendo o AR(1) o quarto melhor modelo, seguido da combinação 5. O modelo AR(1), assumido como benchmarking, portanto, ficou, no melhor caso, como o quarto melhor modelo, quando se avalia a performance nos últimos 48 meses.

Vale ressaltar que, nos últimos 36 meses, o modelo combinado 9 reduziu as métricas tradicionais de erros, incluindo MSE, RMSE, MAE e MAPE, com relação aos valores do ARMA e ARMAX2. Com base em MSPE, o modelo 9 e ARMAX2 apresentaram mesma performance e apenas com base no RSPE, o ARMAX2 tem uma vantagem desprezível. A melhoria de desempenho gerada pela combinação 9 está associada ao conceito de diversificação de riscos, mencionado anterioramente no capítulo 2.

Outro aspecto importante foi a introdução das duas variáveis exógenas no ARMAX2, quais sejam, (i) estoques globais de alumínio e (ii) preço futuro de três meses de alumínio primário. Dias de estoque é uma variável fundamental na indústria global de alumínio e que atua como um indicador do nível de preços.

\subsubsection{Comparação de Modelos por meio do Model Confidence Set}

A Tabela 36 compara o desempenho preditivo dos modelos através do MCS para os últimos 60, 48 e 36 meses da amostra. 
Tabela 41 - Comparação dos modelos por meio do MCS

\begin{tabular}{llllll}
\hline $\mathbf{6 0}$ meses & \multicolumn{3}{l}{$\mathbf{4 8}$ meses } & \multicolumn{3}{l}{ 36 meses } \\
\hline ARMA & $1,0000 *$ & ARMAX2 & $1,0000 *$ & COMB_ARMAX & $1,0000 *$ \\
ARMAX2 & $0,2884 *$ & COMB_ARMAX & $0,8345 *$ & ARMAX2 & $0,1784 * *$ \\
COMB_ARMAX & $0,2873 *$ & & & ARMA & $0,1784 * *$ \\
COMB_AIC & $0,2334 *$ & & & \\
AR1 & $0,2168 *$ & & & \\
ARMAX1 & $0,1063 * *$ & & & \\
Estrutural & $0,1063 * *$ & & & \\
COMB_AVG1 & $0,1063 * *$ & & & \\
COMB_AVG2 & $0,1063 * *$ & & & \\
COMB_OLS & $0,1063 * *$ & & & \\
COMB_RMSE & $0,1063 * *$ & & & \\
COMB_NERLS & $0,1063 * *$ & & & \\
COMB_ERLS & $0,1063 * *$ & & & \\
COMB_NRLS & $0,1063 * *$ & & & & \\
\hline
\end{tabular}

Para os 36 meses mais recentes (outubro/2011 a setembro/2014), a combinação 9 (COMB_ARMAX, que inclui ARMA e ARMAX2 pelo método OLS) apresentou o maior pvalor MCS $(1,000)$, e ficou isolado como o melhor modelo a um nível de significância de 0,10. Os dois outros modelos equiparáveis e abaixo da combinação 9 foram o ARMAX2 e ARMA com p-valor MCS de 0,1784 .

Para os 48 meses mais recentes (outubro/2010 a setembro/2014), o conjunto de modelos equiparáveis ao nível descritivo MCS de 0,10 incluiu o ARMAX2 e COMB_ARMAX. O ARMAX apresentou o maior nível descritivo MCS, seguido da combinação, com nível descritivo MCS 0,8345.

Para os 60 meses mais recentes (outubro/2009 a setembro/2014), o MCS incluiu 5 modelos com performance de previsão equivalente a um nível de confiança de 0,10 do MCS, indicados abaixo. Todos os demais modelos apresentaram desempenho inferior, com nível descritivo do MCS de 0,1063.

- $\quad$ ARMA

- $\quad$ ARMAX2

- COMB_ARMAX2 
- $\quad$ COMB_AIC

- $\quad \operatorname{AR}(1)$

A combinação ARMA/ARMAX (COMB_ARMAX) foi o único modelo que esteve entre os melhores conjuntos avaliados pelo MCS para os três períodos analisados. Para os três anos mais recentes, essa combinação foi o único modelo com maior desempenho preditivo, e nenhum outro teve performance comparável a um nível descritivo MCS de 0,10.

Tal fato indica que a introdução das variáveis exógenas de preço futuro de alumíno de três meses e do nível de estoques globais de alumínio primário foi um diferencial na melhoria da acuracidade.

A Figuras 48 apresenta a importância da baixa taxa de juros no financiamento e aumento de estoques, co-variável relevante para aumentar a acuracidade na previsão de preços de alumínio.

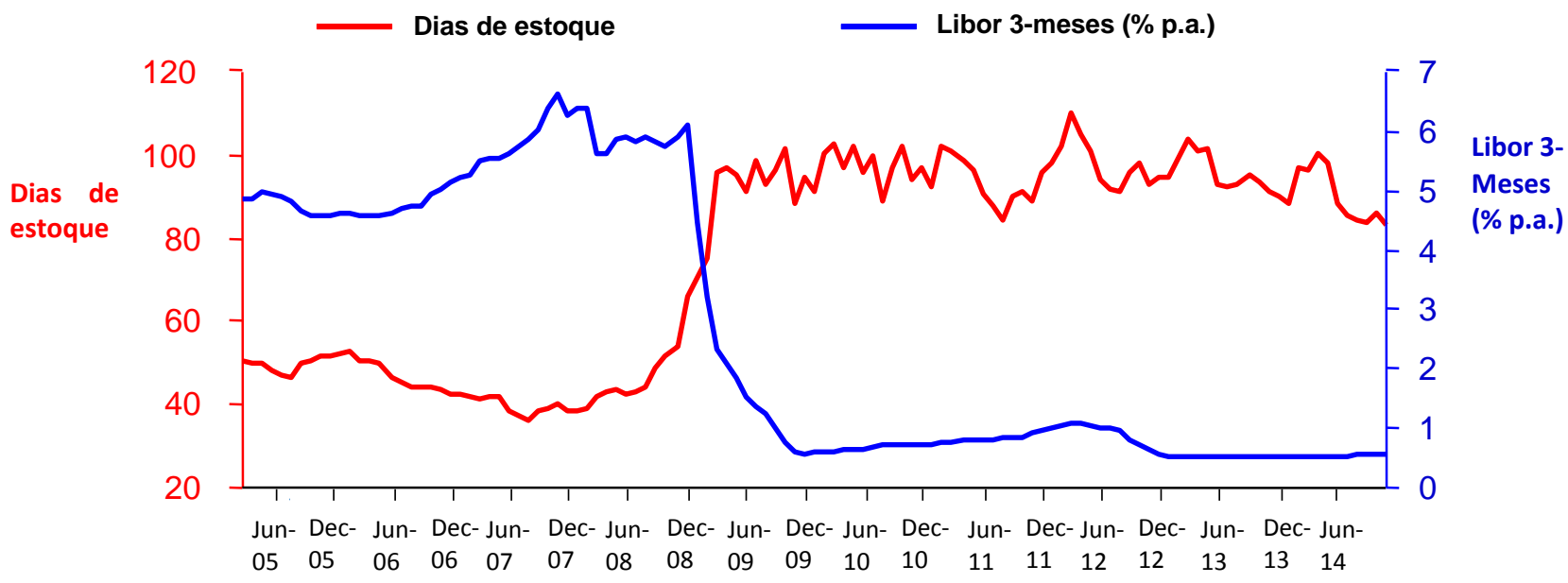

Figura 48 - Evolução de dias de estoques globais de alumínio e taxas de juros 


\section{CONCLUSÃO}

As contribuições desse trabalho incluem três aspectos principais: primeiro, uso de combinações de previsões e de seleção de modelos por meio do Model Confidence Set (MCS), o qual não foi encontrado na literatura para previsões de preços de alumínio. Tal abordagem constituiu um estudo inovador na indústria global de alumínio, com melhorias na acuracidade e seleção de modelos em relação aos métodos tradicionais.

Segundo, foram propostos dois modelos ARMAX, cuja abordagem também não foi encontrada na literatura para projeção de preços de alumínio. O estudo demonstrou a relevância do preço futuro de três meses e dos estoques mundiais para aumentar a acuracidade das previsões.

Terceiro, o trabalho demonstrou que a combinação ótima para previsão 12 meses à frente para preços de alumínio foi o modelo que incluiu ARMA e ARMAX, ajustado por mínimos quadrados ordinários. Essa combinação ótima apresentou um intervalo de confiança pequeno, abaixo de US\$100/t e equivalente a 5\% da média global da amostra completa analisada. Esse conclusão é relevante para tomada de decisões para produtores (como decisões de investimentos) e consumidores (como estratégias de suprimento de matérias-primas), bem como para participantes do mercado financeiro (financiamento de estoques de alumínio) e governos (política industrial).

Sugestões de temas para estudos futuros e de extensão do presente trabalho incluem:

- Combinação de modelos com foco nas densidades de probabilidade de preços de alumínio, a fim de estimar a probabilidade de que os preços fiquem acima ou abaixo de um patamar, ou dentro de uma faixa, o que é relevante para tomada de decisões estratégicas e táticas da indústria

- Modelos de combinação para dados de alta frequência capazes de aumentar acuracidade das previsões de preços em relação aos métodos convencionais de volatilidade

- Determinação de indicadores antecedentes, com base em combinações de variáveis independentes, para aumentar a previsibilidade de choques estruturais, formulação de cenários e suportar tomada de decisão para governos e setores da cadeia de alumínio 


\section{REFERÊNCIAS BIBLIOGRÁFICAS}

ADAMS, F.; VIAL, J. Explaining Recent Metals Price Swings. Resources Policy, 14, p.8596, 1988.

ALFIOLI, M., TIMMERMANN, A. Persistence of Forecasting Performance and Combination Strategies. Journal of Econometrics, 2006

ANDREWS, R. Forecasting performance of structural time series models. Journal of Business and Economics Statistics, v.12, p.129-133, 1994.

BALTAR, B. Análise temporal dos preços da commodity cobre usando o modelo de Box \& Jenkins. 2009. Dissertação - PUC, Rio de Janeiro, 2009.

BARKOULAS, J.; LABYS, W.; ONOCHIE, J. Fractional Dynamics in International Commodity Prices. Journal of Future Markets, v.17, n.2, p.161-89, 1997.

BATES, J. M.; GRANGER, C. W. J. The Combination of Forecasts. Operations Research Quarterly, 20, p.451-68, 1969.

BERNARD, J.T. et al. Forecasting Aluminum Prices: GARCH, Jumps and MeanReversion. Institut de Finance Mathematique de Montreal, October 24, pp.1-15, 2005

BOX, G.; JENKINS, G.; REINSEL, G. Time Series Analysis, Wiley, 2008

BRUGGEMANN, R., LUTKEPOHL, H. Forecasting Contemporaneous Aggregates with Stochastic Aggregation Weights, International Journal of Forecasting, Elsevier, vol. 29(1), pages 60-68, 2013

BUNN, D.W., Statistical Efficiency in the Linear Combination of Forecasts. International Journal of Forecasting, v. 1, p. 151-163, 1985

CASHIN, P., MCDERMOTT, C. Long-run behavior of commodity prices: small trends and big variability. IMF Staff Papers, v.49, n.2, p.177-217, 2002

CHAN, C., KINGSMAN, B., WONG, H. Determining when to update the weights in combined forecasts for product demand - an application of the CUSUM technique, European Journal of Operational Research, c. 153, issue 3, 16, p. 757-768, 2004

CLARK, T., McCRACKEN, Averaging Forecasts from VARs with Uncertain Instabilities. Journal of Applied Econometrics, v. 06-12, 2009

CLEMEN, R.T. Combining Overlapping Information, Management Science, v.33, p.373-380, 1987

CHRISTOFFERSEN, P., DIEBOLD, F. Optimal Prediction under Asymmetric Loss. Economic Theory, v. 13, p. 808-817, 1997 
DIEBOLD, F.; MARIANO, R. S. Comparing Predictive Accuracy. Journal of Business \& Economic Statistics, 13, p.253-63, 1995.

DOOLEY, G.; LENIHAN, H. An assessment of time series methods in metal price forecasting. Resources Policy, 30, p.208-17, 2005.

DRIEHUIS, W. Primary Commodity Prices: Analysis and Forecasting. Rotterdam, Rotterdam University Press, 1976

DUNCAN, R.C. The Outlook of Primary Commodities, Staff Working Paper n. 11, World Bank, Washington D.C., 1984

ELIOTT, G., TIMMERMANN, A. Optimal Forecast Combinations under General Loss Functions and Forecast Error Distributions. Journal of Econometrics, v. 122, p. 147-149, 2004

ELIOTT, G., TIMMERMANN, A. Optimal Forecast Combinations Weights under Regime Switching. International Economic Review, v. 46, p. 1081-1102, 2005

ENGLE, R., GRANGER, C., KRAFT, D. Combining Competing Forecasts of Inflation using a Bivariate ARCH Model. Journal of Economic Dynaics and Control, 8, 151-165, 1984

EVANS, M. Practical Business Forecasting, Wiley, 2003

FERRETTI, I. Prices and Production Cost in Aluminium Smelting in the Short and the Long Run, Departamento de Economía de la Empresa, Universidad Carlos III de Madrid, September 2003

FAN, W. G.; DENG, F. Variable Weight Combining Forecasts Based on Forecasting Error. International Conference on Control and Automation 5: 1610-1613, 2007

FIGLEWISKI, S. Optimal Aggregation of Money Supply Forecasts: Accuracy, Profitability and Market Efficiency, Journal of Finance, 28, 695-710, 1983

GEMAN, H., SMITH, W. Theory of Storage, Inventory and Volatility in the LME Base Metals, Birkbeck, University of London \& ESCP Europe, Resources Policy, 2012

GILBERT, C. Modelling Market Fundamentals: A Model of the Aluminium Market, Journal of Applied Econometrics, Vol. 10, No. 4, pp. 385-410, 1995

GRANGER, C.W. J. Forecasting in Business and Economics. 2.ed., San Diego, 1989

GRANGER, C.W., RAMANATHAN, R. Improved Methods of Combining Forecasts. Journal of Forecasting, 19, 537-560, 1984

HANSEN, P.; LUNDE, A.; NASON, J. The Model Confidence Set. Econometrica, v.79, n.2, p.453-97, mar. 2011.

HARVEY, A. Forecasting, Structural Time Series Models and the Kalman Filter. Cambridge University Press, 1989. 
HARVEY, A. Time Series Models, $2^{\text {nd }}$ edition, Cambridge, The MIT Press, 1994

HARVEY, A. The Econometric Analysis of Time Series, The MIT Press, 1999

HARVEY, A.; JAEGER, A. The effects of Seat Belt Legislation on British Road Casualties: A Case Study in Structural Time Series Modeling. Journal of the Royal Statistical Society, Ser. A., 149, p.187-227, 1993.

HOJMAN, D. An Econometric Model of the Bauxite-Aluminum Economy, Resources Policy, p. 87. June 1981

HSIAO, C., WAN, S.K. (2014). Is There an Optimal Forecast Combination? Journal of Econometrics, 178:294-309, 2014

ISSLER, J., LIMA, R. A Panel Data Approach to Economic Forecasting: The Bias-Corrected Average Forecast, Journal of Econometrics 152 (2), 153-164, 2009

ISSLER, J.; RODRIGUES, C. Comparing forecast accuracy of different models for prices of metal commodities. The Economics and Econometrics of Commodity Prices Conference, EPGE. Rio de Janeiro: Getúlio Vargas Foundation, 2012.

KIM, J. R. K. Combining forecasts using optimal combination weight and generalized autoregression. Journal of Forecasting 27: 419-432, 2008

KOLASSA, S. Combining Exponential Smoothing Forecasts using Akaike Weights, International Journal of Forecasting 27 (2), 238-251, 2011

KOUASSI, E.; LABYS, W.; COLYER, D. The Structural Time Series Approach to Forecasting Commodity Prices, West Virginia University, Morgantown, 1998.

LABYS, W., GRANGER, C. Modeling and Forecasting Primary Commodity Prices, Ashgate, 2006

LABYS, W.; KOUASSI, E. Ciclicality, Stationarity and the Structural Forecasting of Commodity Prices, Working Paper, Natural Resource Economics Program, West Virginia University, 2004.

LABYS, W., POLLACK, P.K. Commodity Models for Forecasting and Policy Analysis, New York, Nichols Publishing, 1984

LAM, K., MUI, H., YUEN, M. Note on Minimizing Absolute Percentage Error in Combined Forecasts. Computers \& Operations Research, v. 8, issue 11, p. 1141-1147, 2001

LAURENT, S.; VIOLANTE, F. Volatility forecasts evaluation and Comparison. WIREs Comp Stat, 4, p.1-12, 2012.

LEAMER, E. Specification Searches: Ad Hoc Inference with Nonexperimental Data. New York: Wiley, 1978. 
LEAMER, E. Let's Take the Con out of Econometrics.American Economic Review, 73, p.3143, 1983.

LEUTHOLD, R.M., MacCORMICK, A., SCHMITZ, A., WATTS, D.G. Forecasting Daily Hog Prices and Quantities: A Study of Alternative Forecasting Techniques, Journal of the American Statistical Association, v. 65, p. 65-90, 1970

LIANG, K. Y.; LEE, J. C.; SHAO, K. S. H. On the Distribution of the Inverted Linear Compound of Dependent F-Variates and its Application to the Combination of Forecasts. Journal of Applied Statistics 33: 961-973, 2006

LO, A.; MACKINLEY, C. Data Snooping Biases in Tests of Tinancial Asset Pricing Models. Review of Financial Studies, 3, p.431-68, 1990.

LOVELL, M. C. Data Mining. Review of Economics and Statistics, 45, p.1-12. 1983.

LUTKEPOHL, H. New Introduction to Multiple Time Series. Springer, 2007

MAKRIDAKIS, S. et al. The Accuracy of Extrapolation Methods: results of a forecasting competition. J. Forecasting, v.1, n.11, p.1-153, 1982.

MAKRIDAKIS, S. et al. The Accuracy of Major Extrapolation. London: Wiley, 1983.

MAKRIDAKIS, S. Why Combining Works? International Journal of Forecasting, v. 5, p. 601-603, 1989

MAKRIDAKIS, S., WINKLER, R., Averages of Forecasts: Some Empirical Results, Management Science, v. 29, p. 987-996, 1983

MASOUD, M. Structural and Multivariate Time Series Models: Comparative Application in Commodity Markets, Disserration, West Virginia University, 1988

MAYER, T. "Economics as a hard science: realistic goal or wishful thinking?" Economic Inquiry, 18, p.165-78, 1980.

MILLER, R. G. Simultaneous Statistical Inference. New York: Springer-Verlag, 1981.

MILLS, T. Modeling trends and cycles in economic time series. New York: Palgrave, 2003.

MUTAFOGLU, T., TOKAT, E., TOKAT, H. Forecasting Precious Metal Price Movements using Trader Positions, Resources Policy, v. 37, p. 273-280, 2012

NELSON, C.R. The Prediction Performance of the FRB-MIT-PENN Model of the US Economy, American Economy Review, v. 62, p. 902-17, 1972

NEWBOLD, P.; GRANGER, C. W. J. Experience with forecasting univariate time series and the combination of forecasts. Journal of the Royal Statistical Society, Series A, 137, p.13146, 1974. 
PERRON, P. The Great Crash, the Oil Price Shock and the Unit Root Hypothesis. Econometrica, 55, p.277-309,1989.

PESARAN, M.H., TIMMERMANN, A. Selection of Estimation Window in the Presence of Breaks, Department of Economics, University of California, San Diego, 2005

REN, Y., RU, Y., Application of ARMA Model in Forecasting Aluminum Price, Applied Mechanics and Materials Vols. 155-156, pp 66-71, 2012

SAS Forecast Server Procedures 13.2, User's Guide, SAS Institute Inc., 2014

SLUTSKY, E. The Summation of Random Causes as a Source of Cyclic Processes. Econometrica, vol. 5, p. 105-146, 1927

SMITH, J.; WALLIS, K. Simple explanation of the forecast combination puzzle. Oxford Bulletin of Economics and Statistics,v.71, n.3, p.331-355, 2009.

SOW, T. Multivariate Forecasting of Mineral Commodities Prices: Implications for Natural Resource Scarcity, PhD Thesis, Dept. of Mining \& Geological Engineering, University of Arizona, 1996.

STOCK, J.H., WATSON, M. A Comparison of Linear and Nonlinear Univariate Models for Forecasting Macroeconomic Time Series, in: Engle, R.F., White, H. (eds), Cambridge University Press, Cambridge, 2001

STOCK, J.H., WATSON, M. Combination of Forecasts of Output Growth in a Seven-Country Dataset, Journal of Forecasting, 23, 405-430, 2004

STOFFER, D.; SCHUMWAY, R. Time Series Analysis and Its Applications. Springer, 2006.

TIMMERMANN, A. Forecast combinations. In: WLLIOTT, G.; GRANGER, C. W. J.; TIMMERMANN, A. Handbook of Economic Forecasting, North-Holland, 2006.

TIMMERMAnN, A., GARGANO, A. Predictive Dynamics in Commodity Prices, Seminar on Commodity Price Forecasting, FGV/RJ, August 2012

WHITE, H. Reality Check for Data Snooping. Econometrica, v.68, n.5, p.1097-126, set. 2000.

WINKLER, R.L. Combining Forecasts: A Philosofical Basis and Some Current Issues. International Journal of Forecasting, 5, p. 605-609, 1989

WOOLDRIDGE, J. Econometric Analysis of Cross Section and Panel Data, The MIT Press, 2010

YULE, G. U. On a Method of Investigating Periodicities in Disturbed Series, with Special Reference to Wolfer's Sunspot Numbers. Philosophical Transactions, vol. 22-A, 1927 


\section{ANEXO A - Programa SAS: Combinações de Modelos}

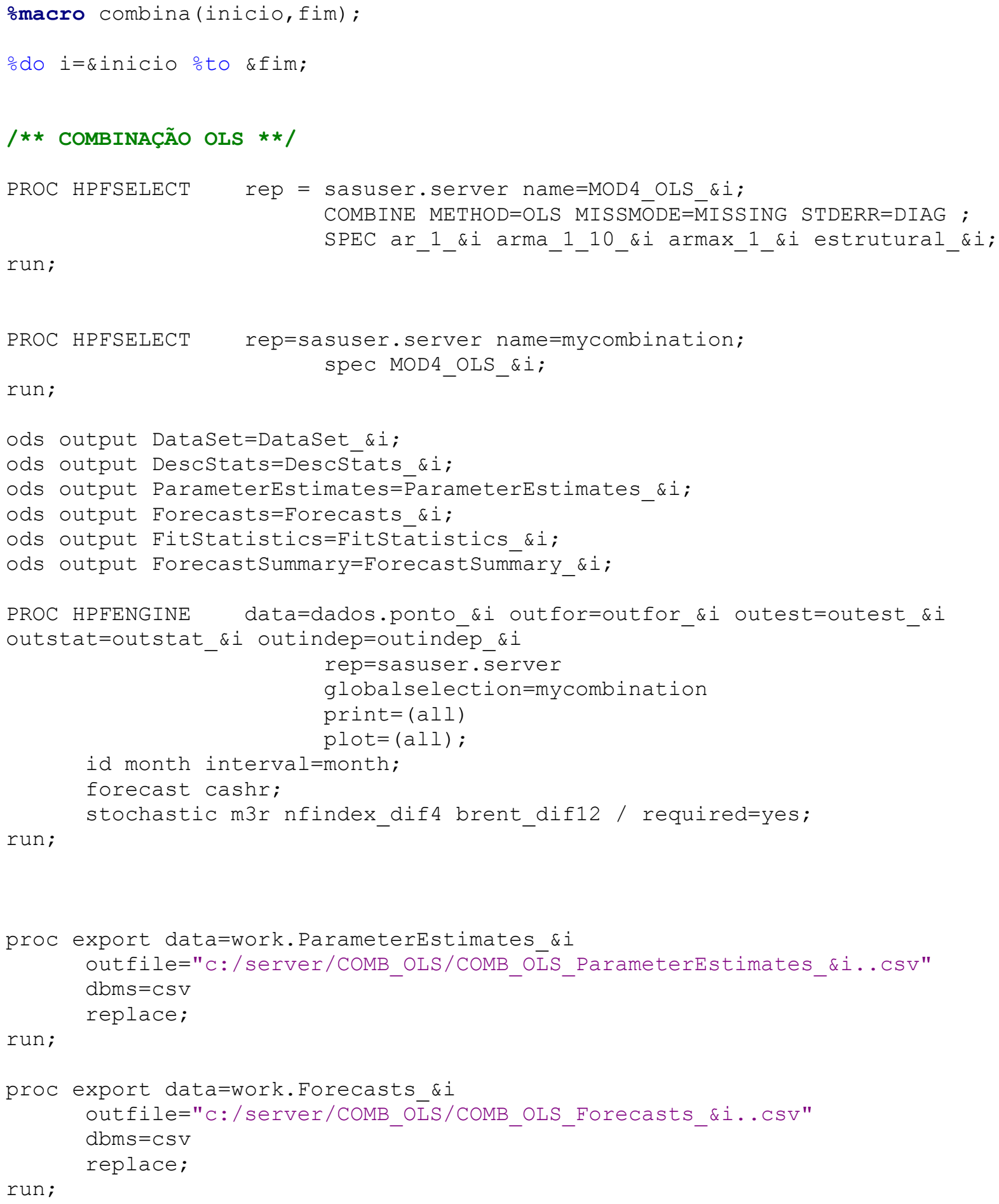

run;

PROC HPFSELECT rep=sasuser. server name=mycombination; spec MOD4_OLS_\&i;

run;

ods output DataSet=DataSet \&i;

ods output DescStats=DescStats_i;

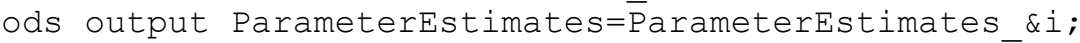

ods output Forecasts $=$ Forecasts \&i;

ods output Fitstatistics=Fitstatistics_\&i;

ods output ForecastSummary=ForecastSummary_\&i;

PROC HPFENGINE data=dados.ponto \&i outfor=outfor \&i outest=outest \&i outstat=outstat_\&i outindep=outindep_\&i

rep=sasuser. server

globalselection=mycombination

print $=(\operatorname{all})$

id month interval=month;

plot=(all);

forecast cashr;

stochastic m3r nfindex_dif4 brent_dif12 / required=yes;

run;

proc export data=work.ParameterEstimates_\&i

outfile="C:/server/COMB OLS/COMB OLLS ParameterEstimates \&i..csv"

$\mathrm{dbms}=\mathrm{CsV}$

replace;

run;

proc export data=work.Forecasts_\&i

outfile="C:/server/COMB_OLS/COMB_OLS_Forecasts_\&i...CsV"

doms $=\mathrm{Csv}$

replace;

run;

/** COMBINAÇÃO AICC **/

PROC HPFSELECT rep = sasuser.server name=MOD4_AICC_\&i;

COMBINE METHOD=AICC ;

SPEC ar_1_\&i arma_1_10_\&i armax_1_\&i estrutural_\&i; 
run;

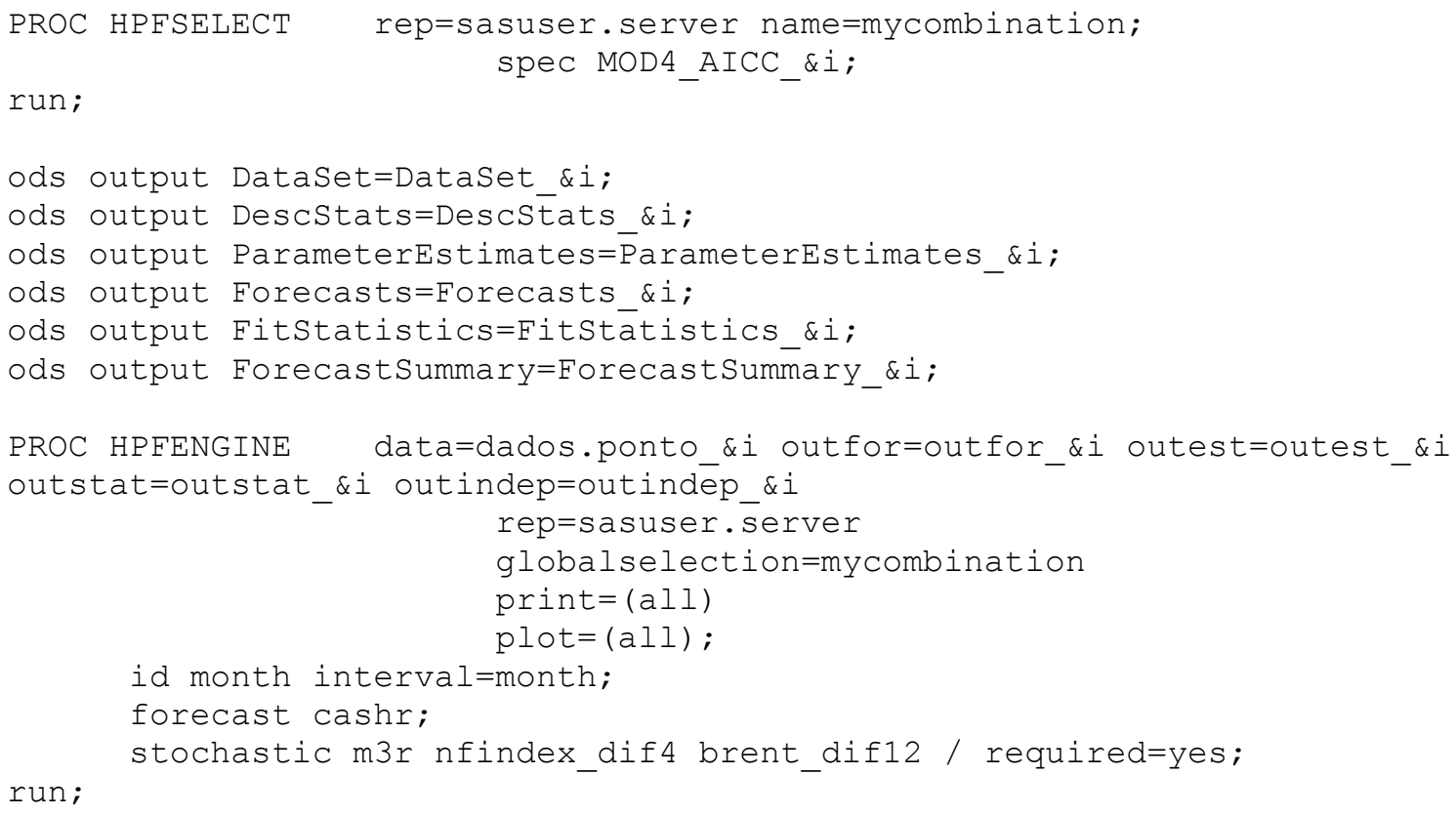

proc export data=work. ParameterEstimates \&i outfile="C:/server/COMB_AICC/COMB_ĀICC_ParameterEstimates_\&i..CSV" $\mathrm{dbms}=\mathrm{CSV}$ replace;

run;

proc export data=work.Forecasts_\&i

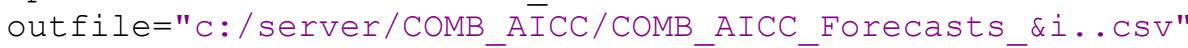
$\mathrm{dbms}=\mathrm{Cs} \mathrm{V}$ replace;

run;

\section{/** COMBINAÇÃO PELA MÉDIA **/}

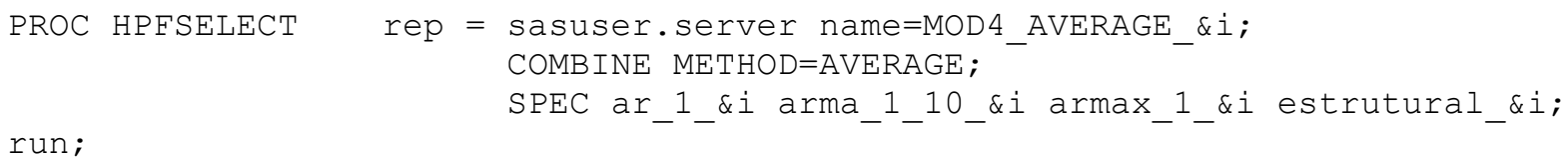

run;

PROC HPFSELECT rep=sasuser. server name=mycombination; spec MOD4_AVERAGE_\&i;

run;

ods output DataSet=DataSet \&i;

ods output DescStats=DescStats_\&i;

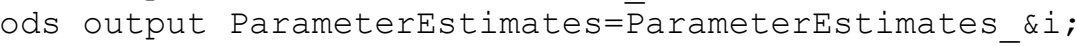

ods output Forecasts=Forecasts_di;

ods output Fitstatistics=Fitstatistics_\&i;

ods output ForecastSummary=ForecastSummary_\&i; 
PROC HPEENGINE data=dados.ponto_\&i outfor=outfor_\&i outest=outest_\&i outstat=outstat_\&i outindep=outindep_\&i

rep=sasuser. server

globalselection=mycombination print $=(a l l)$ plot $=($ all $)$;

id month interval=month;

forecast cashr;

stochastic m3r nfindex_dif4 brent_dif12 / required=yes;

run;

proc export data=work.ParameterEstimates_\&i

outfile="c:/server/COMB_AVERAGE/COM̄B AVERAGE_ParameterEstimates_\&i..c

SV"

$\mathrm{dbms}=\mathrm{CsV}$

replace;

run;

proc export data=work. Forecasts_\&i

outfile="c:/server/COMB_AV̄ERAGE/COMB_AVERAGE_Forecasts_\&i..csv"

$\mathrm{dbms}=\mathrm{CSV}$

replace;

run;

/** COMBINAÇÃO POR ERLS **/

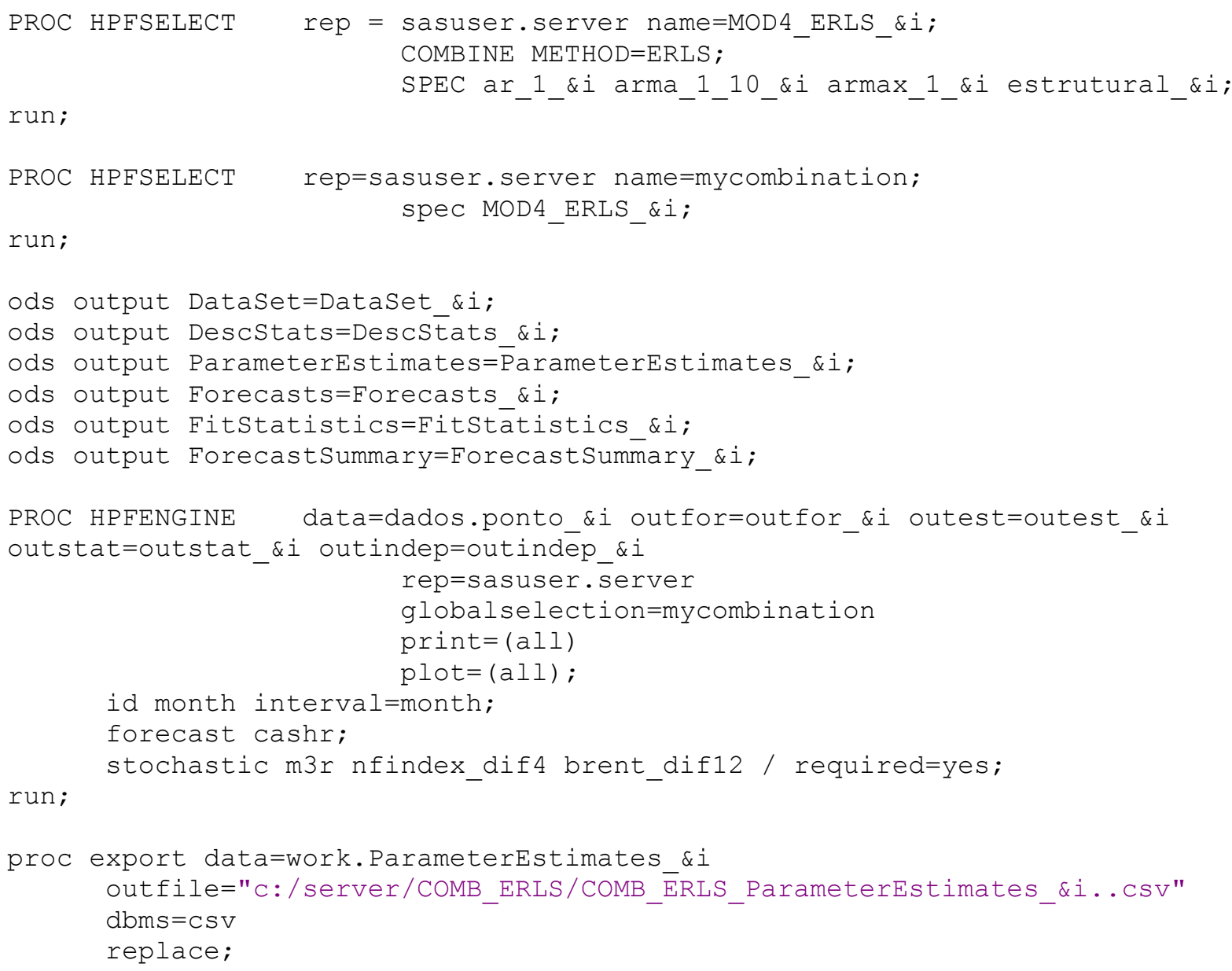

run;

ods output DataSet=DataSet_\&i;

ods output DescStats=DescSt̄ats_\&i;

ods output ParameterEstimates $=\bar{P}$ arameterEstimates_\&i;

ods output Forecasts=Forecasts_\&i;

ods output Fitstatistics=Fitstatistics_\&i;

ods output ForecastSummary=ForecastSummary_\&i;

PROC HPFENGINE data=dados.ponto_\&i outfor=outfor_\&i outest=outest_\&i outstat=outstat_\&i outindep=outindep_\&i

rep=sasuser. server

globalselection=mycombination

print $=($ all $)$

id month interval=month;

plot $=($ all $)$;

forecast cashr;

run;

stochastic m3r nfindex_dif4 brent_dif12 / required=yes;

proc export data=work.ParameterEstimates_\&i

outfile="c:/server/COMB_ERLS/COMB_ERLS_ParameterEstimates_\&i..csv"

dbms $=\mathrm{Csv}$

replace;

run; 


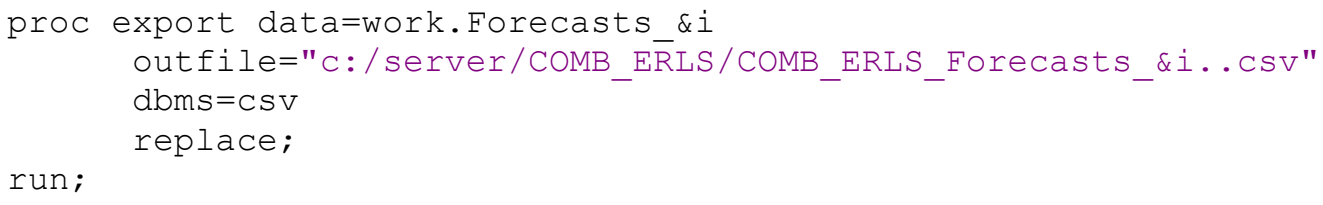

run;

ods output DataSet=DataSet_\&i;

ods output DescStats=DescStats_\&i;

ods output ParameterEstimates= $\bar{P}$ arameterEstimates \&i;

ods output Forecasts=Forecasts_\&i;

ods output Fitstatistics=Fitstatistics_\&i;

ods output ForecastSummary=ForecastSummary_\&i;

PROC HPFENGINE data=dados.ponto_\&i outfor=outfor_\&i outest=outest_\&i outstat=outstat_\&i outindep=outindēp_\&i

rep=sasuser. $\overline{\text { server }}$

globalselection=mycombination

print $=($ all $)$

id month interval=month;

plot $=($ all $)$;

forecast cashr;

stochastic m3r nfindex_dif4 brent_dif12 / required=yes;

run;

proc export data=work.ParameterEstimates_\&i

outfile="c:/server/COMB NERLS/COMB NERLS ParameterEstimates \&i..csv" $\mathrm{dbms}=\mathrm{Cs} \mathrm{V}$

replace;

run;

proc export data=work.Forecasts_\&i

outfile="c:/server/COMB_NERLS/COMB_NERLS_Forecasts_\&i...CSV"

doms $=\mathrm{Cs} \mathrm{V}$

replace;

run;

/** COMBINAÇÃO POR NRLS **/

PROC HPFSELECT rep = sasuser.server name=MOD4_NRLS_\&i; COMBINE METHOD=NRLS;

SPEC ar_1_\&i arma_1_10_\&i armax_1_\&i estrutural_\&i;

run;

PROC HPFSELECT rep=sasuser. server name=mycombination;

spec MOD4_NRLS_\&i;

run; 


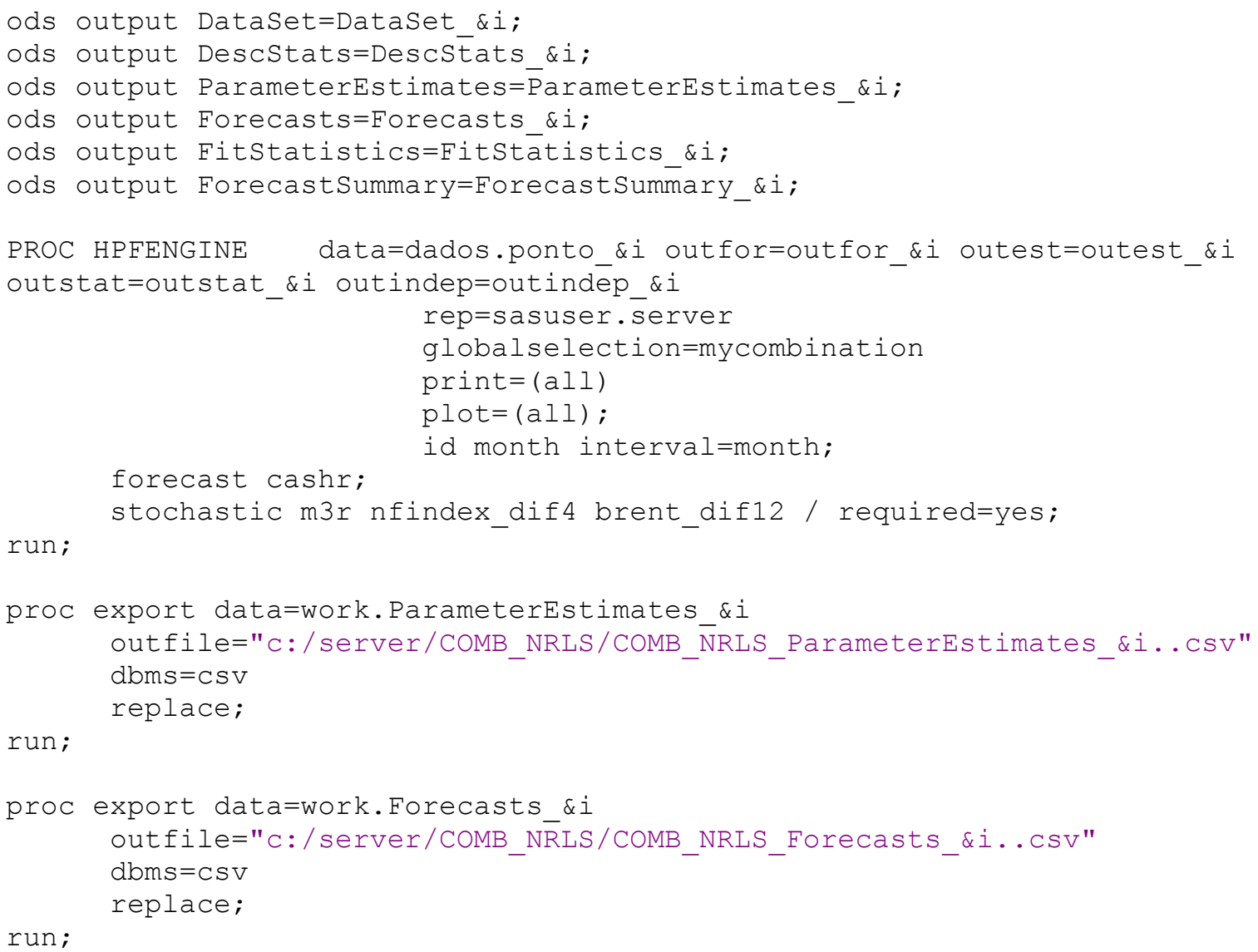

/** COMBINAÇÃO POR RMSE **/

PROC HPFSELECT rep = sasuser.server name=MOD4 RMSEWGT_\&i; COMBINE METHOD=RMSEWGT; SPEC ar_1_\&i arma_1_10_\&i armax_1_\&i estrutural_\&i;

run;

PROC HPFSELECT rep=sasuser. server name=mycombination; spec MOD4_RMSEWGT_\&i;

run;

ods output DataSet=DataSet \&i;

ods output DescStats=DescSt̄ats \&i;

ods output ParameterEstimates $=\bar{P}$ arameterEstimates_\&i;

ods output Forecasts=Forecasts_\&i;

ods output Fitstatistics=Fitstatistics \&i;

ods output ForecastSummary=ForecastSummary_\&i;

PROC HPFENGINE data=dados.ponto_\&i outfor=outfor_\&i outest=outest_\&i outstat=outstat_\&i outindep=outindēp_\&i

rep=sasuser. server

globalselection=mycombination

print $=($ all $)$

plot= (all);

id month interval=month;

forecast cashr;

stochastic m3r nfindex_dif4 brent_dif12 / required=yes;

run; 


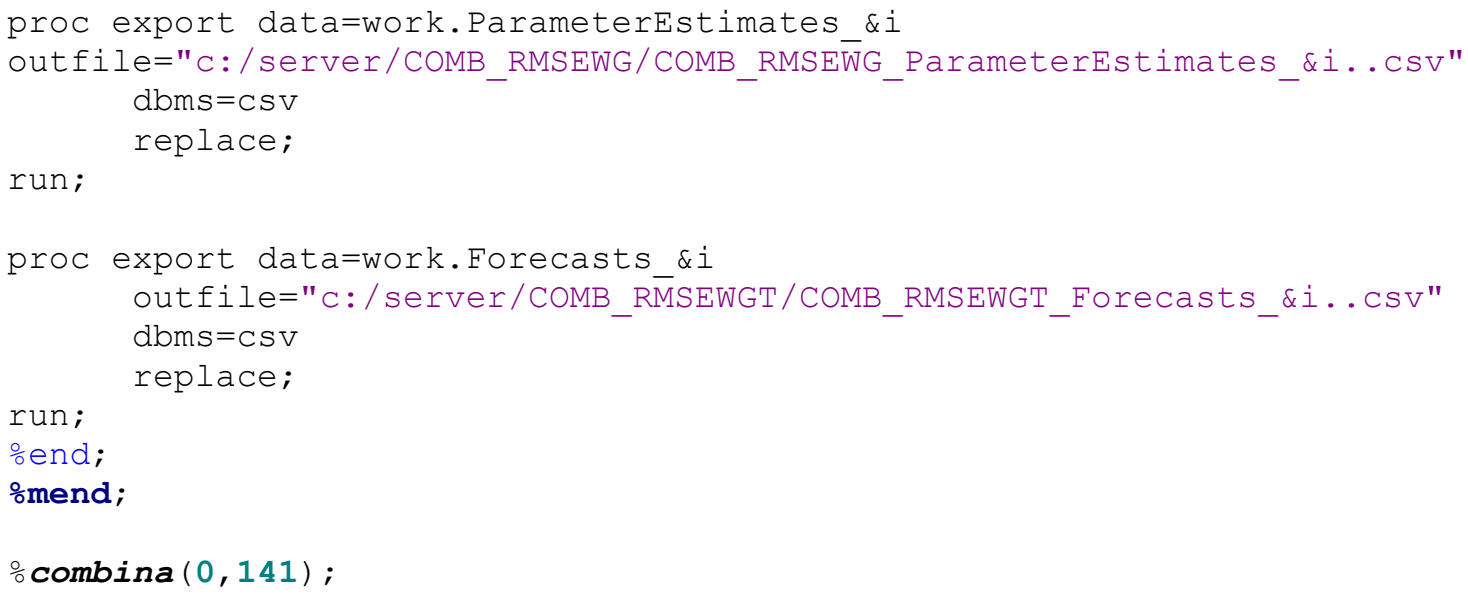

\section{ANEXO B: Programa SAS para Geração de Modelos Individuais - 12 Meses à Frente}

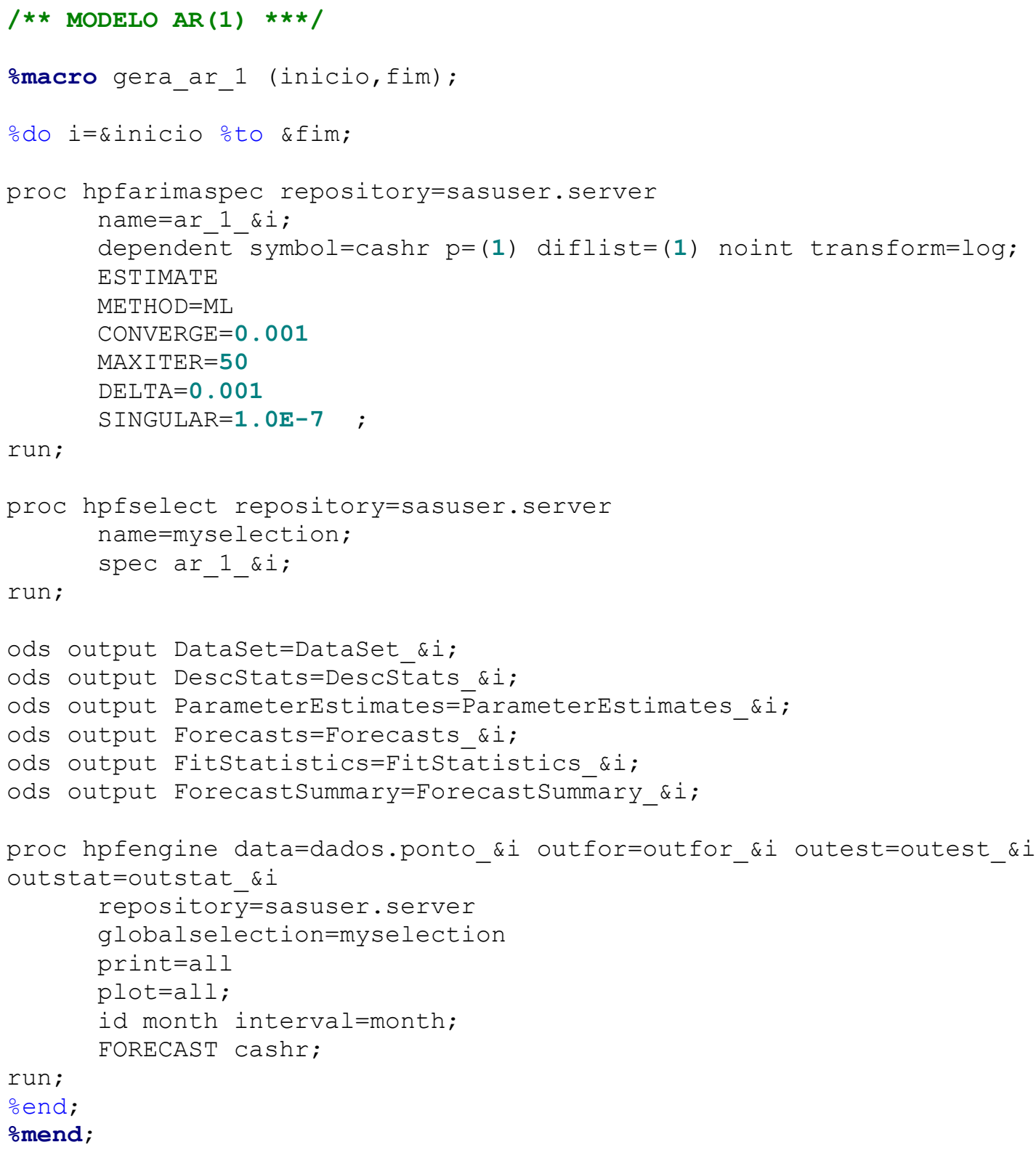




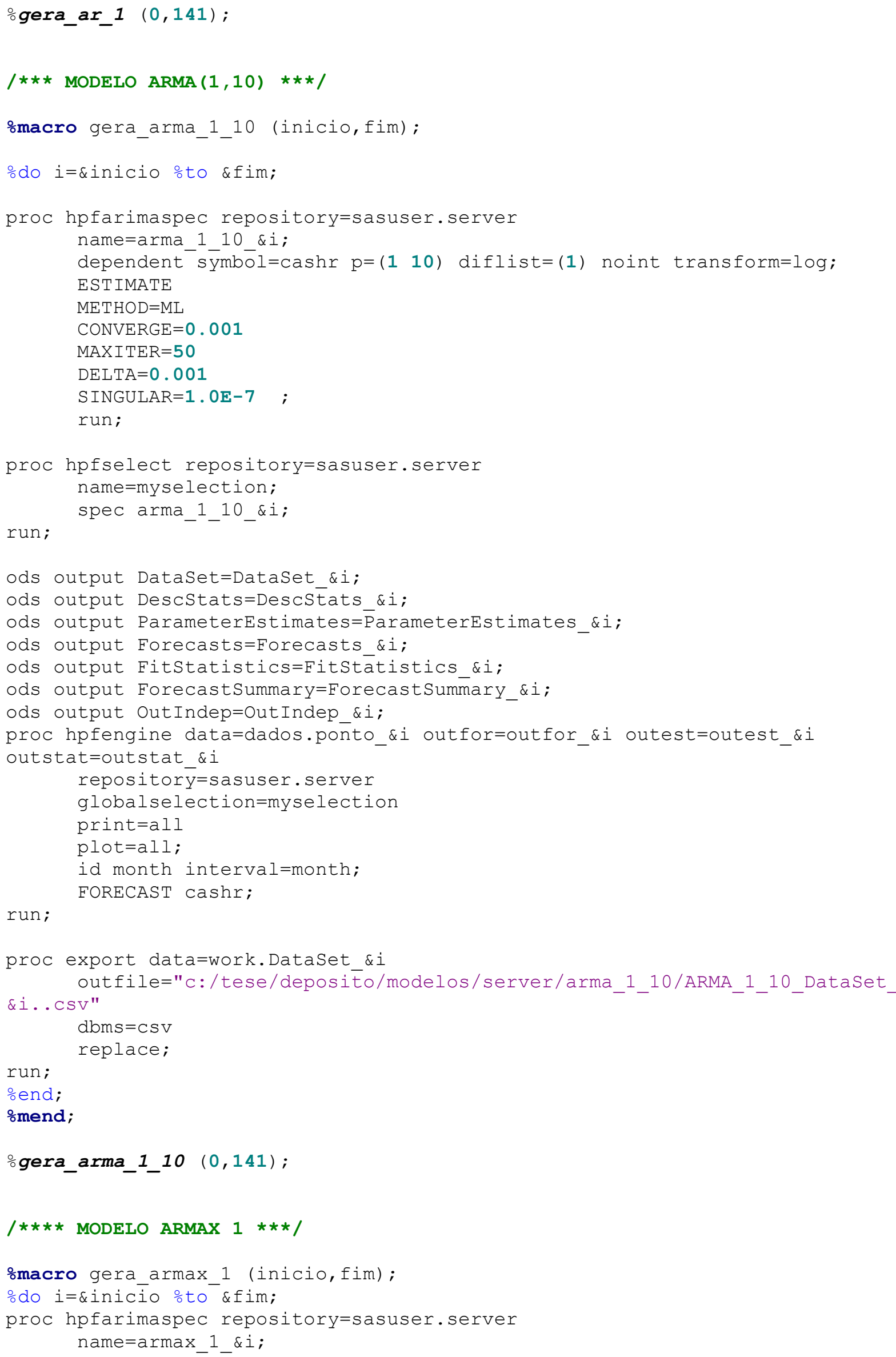




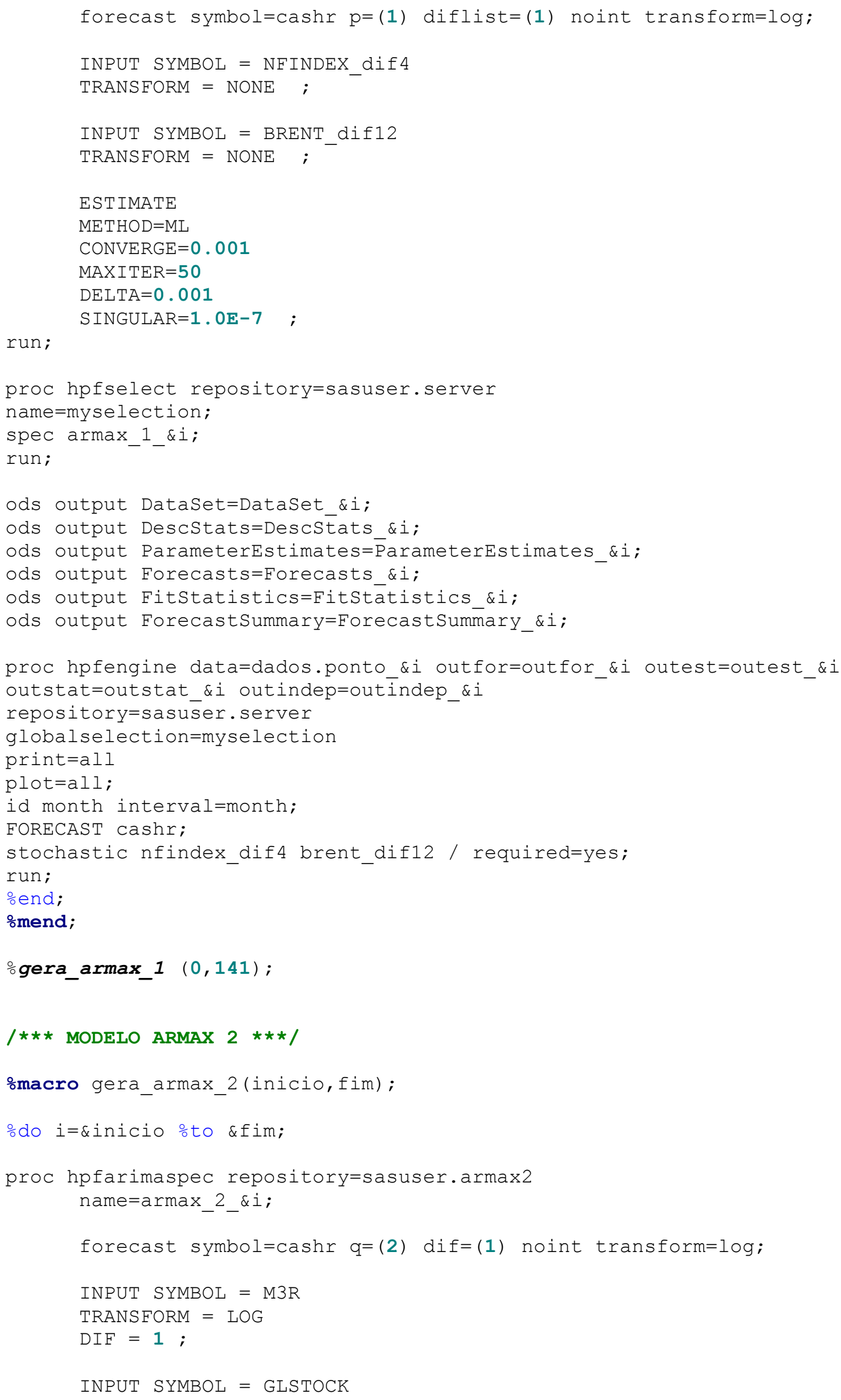




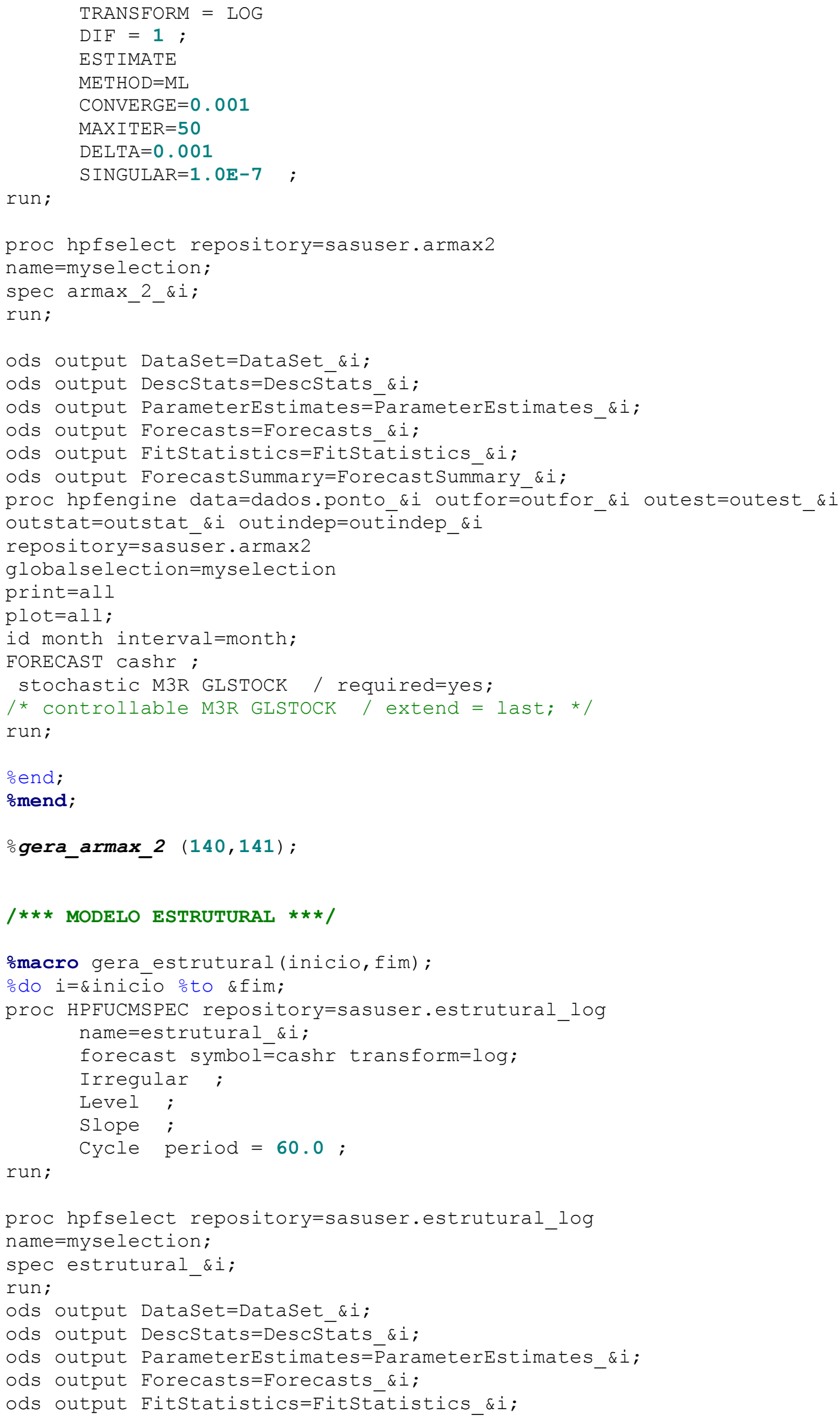


ods output ForecastSummary=ForecastSummary_\&i;

proc hpfengine data=dados.ponto_\&i outfor=outfor_\&i outest=outest_\&i outstat=outstat_\&i

repository=sasuser.estrutural_log

globalselection=myselection

print=all

plot=all;

id month interval=month;

FORECAST cashr;

run;

ond;

omend;

ogera_estrutural $(0,141)$; 